\title{
Dynamics in chemotaxis models of parabolic-elliptic type on bounded domain with time and space dependent logistic sources
}

\author{
Tahir Bachar Issa and Wenxian Shen \\ Department of Mathematics and Statistics \\ Auburn University \\ Auburn University, AL 36849 \\ U.S.A.
}

\begin{abstract}
The current paper considers the dynamics of the following chemotaxis system of parabolicelliptic type with local as well as nonlocal time and space dependent logistic source

$$
\left\{\begin{array}{l}
u_{t}=\Delta u-\chi \nabla(u \cdot \nabla v)+u\left(a_{0}(t, x)-a_{1}(t, x) u-a_{2}(t, x) \int_{\Omega} u\right), \quad x \in \Omega \\
0=\Delta v+u-v, \quad x \in \Omega \\
\frac{\partial u}{\partial n}=\frac{\partial v}{\partial n}=0, \quad x \in \partial \Omega
\end{array}\right.
$$

where $\Omega \subset \mathbb{R}^{n}(n \geq 1)$ is a bounded domain with smooth boundary $\partial \Omega$ and $a_{i}(t, x)(i=0,1,2)$ are locally Hölder continuous in $t \in \mathbb{R}$ uniformly with respect to $x \in \bar{\Omega}$ and continuous in $x \in \bar{\Omega}$. We first prove the local existence and uniqueness of classical solutions $\left(u\left(x, t ; t_{0}, u_{0}\right), v\left(x, t ; t_{0}, u_{0}\right)\right)$ with $u\left(x, t_{0} ; t_{0}, u_{0}\right)=u_{0}(x)$ for various initial functions $u_{0}(x)$. Next, under some conditions on the coefficients $a_{1}(t, x), a_{2}(t, x)$, and $\chi$ and the dimension $n$, we prove the global existence and boundedness of classical solutions $\left(u\left(x, t ; t_{0}, u_{0}\right), v\left(x, t ; t_{0}, u_{0}\right)\right)$ with given nonnegative initial function $u\left(x, t_{0} ; t_{0}, u_{0}\right)=u_{0}(x)$. Then, under the same conditions for the global existence, we show that the system has an entire positive classical solution $\left(u^{*}(x, t), v^{*}(x, t)\right)$. Moreover, if $a_{i}(t, x)(i=0,1,2)$ are periodic in $t$ with period $T$ or are independent of $t$, then the system has a time periodic positive solution $\left(u^{*}(x, t), v^{*}(x, t)\right)$ with periodic $T$ or a steady state positive solution $\left(u^{*}(x), v^{*}(x)\right)$. If $a_{i}(t, x)(i=0,1,2)$ are independent of $x$ (i.e. are spatially homogeneous), then the system has a spatially homogeneous entire positive solution $\left(u^{*}(t), v^{*}(t)\right)$. Finally, under some further assumptions, we prove that the system has a unique entire positive solution $\left(u^{*}(x, t), v^{*}(x, t)\right)$ which is globally stable in the sense that for any given $t_{0} \in \mathbb{R}$ and $u_{0} \in C^{0}(\bar{\Omega})$ with $u_{0}(x) \geq 0$ and $u_{0}(x) \not \equiv 0$,

$$
\lim _{t \rightarrow \infty}\left(\left\|u\left(\cdot, t ; t_{0}, u_{0}\right)-u^{*}(\cdot, t)\right\|_{C^{0}(\bar{\Omega})}+\left\|v\left(\cdot, t ; t_{0}, u_{0}\right)-v^{*}(\cdot, t)\right\|_{C^{0}(\bar{\Omega})}\right)=0 .
$$
\end{abstract}

Moreover, if $a_{i}(t, x)(i=0,1,2)$ are periodic or almost periodic in $t$, then the unique entire positive solution $\left(u^{*}(x, t), v^{*}(x, t)\right)$ is also periodic or almost periodic in $t$.

Key words. Parabolic-elliptic chemotaxis system, logistic source, classical solution, local existence, global existence, entire positive solution, periodic positive solution, almost periodic positive solution, asymptotic behavior.

2010 Mathematics Subject Classification. 35B08, 35B10, 35B15, 35B35, 35B40, 35K57, 35Q92, 92C17. 


\section{Introduction and the statements of the main results}

Chemotaxis refers to the movement of living organisms in response to certain chemicals in their environments. This type of movement exists in many biological phenomena such as bacteria aggregation, immune system response or angiogenesis in the embryo formation and in tumour development. At the beginning of the 1970s, Keller and Segel ([21, 22]) introduced some mathematical models to describe the aggregation of certain types of bacteria. Since then, a variety of mathematical models to describe chemotaxis have been proposed. Systems with chemotactic terms have been used to model not only the mentioned biological processes at the microscopic scale but also population dynamics at the macroscopic scale in the context of life sciences, 'gravitational collapse' in astrophysics, material sciences, etc. A large amount of research has been carried out toward various central problems in chemotaxis models, including global existence of classical/weak solutions with given initial data; finite-time blow-up; pattern formation; existence, uniqueness, and stability of certain special solutions; etc. (see, for instance, [5, 9, 10, 17, 18, 19, 36, 37, 39, 46], etc.).

Consider chemotaxis systems for the time evolution of the densities of one species and one chemoattractant. According to the nature of the equation satisfied by the chemoattractant, such systems can be classified as parabolic-parabolic systems, parabolic-elliptic systems, or parabolicODE systems. Many interesting dynamical scenarios are observed in such chemotaxis systems. For example, it is observed that chemotactic cross-diffusion may exert a strongly destabilizing action in the sense that finite-time blow-up might occur in the following system,

$$
\left\{\begin{array}{l}
u_{t}=\Delta u-\chi \nabla(u \cdot \nabla v), \quad x \in \Omega \\
\tau v_{t}=\Delta v+u-v, \quad x \in \Omega \\
\frac{\partial u}{\partial n}=\frac{\partial v}{\partial n}=0, \quad x \in \partial \Omega,
\end{array}\right.
$$

(see [12, 14, 20, 29, 30] for the parabolic-elliptic case (i.e. $\tau=0$ ) and [13], 49], 48] for the fully parabolic case (i.e. $\tau>0)$ ). It is observed that logistic-type sources may suppress such blow-up phenomena to some extent. More precisely, consider

$$
\left\{\begin{array}{l}
u_{t}=\Delta u-\chi \nabla(u \cdot \nabla v)+u(a-b u), \quad x \in \Omega \\
\tau v_{t}=\Delta v+u-v, \quad x \in \Omega \\
\frac{\partial u}{\partial n}=\frac{\partial v}{\partial n}=0, \quad x \in \partial \Omega
\end{array}\right.
$$

It is shown that solutions of (1.2) with positive initial functions exist globally in time provided that $b$ is sufficiently large relative to $\chi$ (see e.g. [27, 32, 43, 44, 45, 47]). Also, quite rich dynamical features, including spatial pattern formation and spatio-temporal chaos, may be observed in chemotaxis models, including (1.2), at least numerically (see [26, 35]). The reader is referred to [4, 23, 25, 28, 40, 42, 41, 50, etc. for the analytic studies of chemotaxis models with logistic sources. However, many central problems for chemotaxis models with logistic sources, including the possible occurrence of finite time blow up when the logistic damping is not large relative to the chemotaxis sensitivity, are not well understood yet. In particular, there is little study on chemotaxis systems with time and space dependent logistic sources. 
In reality, the environments of many living organisms are spatially and temporally heterogeneous. It is of both biological and mathematical interests to study chemotaxis models with certain time and space dependence. In the present paper, we consider the following chemotaxis system of parabolic-elliptic type with both local and nonlocal heterogeneous logistic source,

$$
\left\{\begin{array}{l}
u_{t}=\Delta u-\chi \nabla(u \cdot \nabla v)+u\left(a_{0}(t, x)-a_{1}(t, x) u-a_{2}(t, x) \int_{\Omega} u\right), \quad x \in \Omega \\
0=\Delta v+u-v, \quad x \in \Omega \\
\frac{\partial u}{\partial n}=\frac{\partial v}{\partial n}=0, \quad x \in \partial \Omega
\end{array}\right.
$$

where $\Omega$ is a bounded subset of $\mathbb{R}^{n}$ with smooth boundary, $u(x, t)$ and $v(x, t)$ represent the population densities of living organisms and some chemoattractant substance, respectively, $\chi \in$ $\mathbb{R}$ is the chemotactic sensitivity, $a_{0}, a_{1}$ are nonnegative bounded functions and $a_{2}$ is a bounded real valued function.

System (1.3) with constant coefficients for both one and two species, was introduced recently in [31] by Negreanu and Tello. As mentioned in [31, the logistic growth describes the competition of the individuals of the species for the resources of the environment and the cooperation to survive. The coefficient $a_{0}$ induces an exponential growth for low density populations and the term $a_{1} u$ describes a local competition of the species. At the time that the population grows, the competitive effect of the local term $a_{1} u$ becomes more influential. The non-local term $a_{2} \int_{\Omega} u$ describes the influence of the total mass of the species in the growth of the population. If $a_{2}>0$, we have a competitive term which limits such growth and when $a_{2}<0$ the individuals cooperate globally to survive. In the last case, the individuals compete locally but cooperate globally and the effects of $a_{1} u$ and $a_{2} \int_{\Omega} u$ balance the system. Note that $(u, v) \equiv(0,0)$ is always a solution of (1.3), which will be called the trivial solution of (1.3). Due to the biological reason, we are only interested in nonnegative solutions, in particular, nonnegative and nontrivial solutions, of (1.3).

In the case that the chemotaxis and nonlocal competition are absent (i.e. $\chi=0$ and $a_{2} \equiv 0$ ) in (1.3), the population density $u(x, t)$ of the living organisms satisfies the following scalar reaction diffusion equation,

$$
\left\{\begin{array}{l}
u_{t}=\Delta u+u\left(a_{0}(t, x)-a_{1}(t, x) u\right), \quad x \in \Omega \\
\frac{\partial u}{\partial n}=0, \quad x \in \partial \Omega .
\end{array}\right.
$$

Equation (1.4) is called Fisher or KPP type equation in literature because of the pioneering works by Fisher ([7]) and Kolmogorov, Petrowsky, Piscunov ([24]) in the special case $a_{0}(t, x)=$ $a_{1}(t, x)=1$, and has been extensively studied (see [3], 15], [33], 38], [51, etc.). The dynamics of (1.4) (and then the dynamics of (1.3) ) is quite well understood. For example, if $a_{0}(t, x) \equiv a_{0}(t)$ and $a_{1}(t, x) \equiv a_{1}(t)$, it is proved in [33] that (1.3) has a unique bounded entire solution, that is positive, does not approach the zero-solution in the past and in the future and attracts all positive solutions. If $a_{0}(t, x)$ and $a_{1}(t, x)$ are positive and almost periodic in $t$, it is proved in 38] that (1.4) has a unique globally stable time almost periodic positive solution.

In the case of constant coefficients with $a_{0}>0$ and $a_{1}-|\Omega|\left(a_{2}\right)_{-}>0$, it is clear that $(u, v) \equiv\left(\frac{a_{0}}{a_{1}+a_{2}|\Omega|}, \frac{a_{0}}{a_{1}+a_{2}|\Omega|}\right)$ is the unique nontrivial spatially and temporally homogeneous steady 
state solution of (1.3), where $|\Omega|$ is the Lebesgue measure of $\Omega$. It is proved in [31] that the condition $a_{1}>2 \chi+\left|a_{2}\right|$ ensures the global stability of the homogeneous steady state (see [43] when $a_{2}=0$ ) and that, if furthermore $a_{2}=0$, the assumption $a_{1}>\frac{n-2}{n} \chi$ ensures the global existence of a unique bounded classical solution $\left(u\left(x, t ; t_{0}, u_{0}\right), v\left(x, t ; t_{0}, u_{0}\right)\right)$ with given nonnegative initial function $u_{0} \in C^{0, \alpha}(\bar{\Omega})$ (i.e. $\left.u\left(x, t_{0} ; t_{0}, u_{0}\right)=u_{0}(x) \geq 0\right)$ (see [43]). It should be pointed that, when $n \geq 3$ and $a_{1} \leq \frac{n-2}{n} \chi\left(\chi>0\right.$ and $\left.a_{2}=0\right)$, it remains open whether for any given nonnegative initial function $u_{0} \in C^{0, \alpha}(\bar{\Omega})$, (1.3) possesses a global classical solution $\left(u\left(x, t ; t_{0}, u_{0}\right), v\left(x, t ; t_{0}, u_{0}\right)\right)$ with $u\left(x, t_{0} ; t_{0}, u_{0}\right)=u_{0}(x)$, or whether finite-time blow-up occurs for some initial data. We mention the works [27], [49], [50] along this direction. It is shown in [27, [50] that in presence of suitably weak logistic dampening (that is, small $a_{1}$ ) certain transient growth phenomena do occur for some initial data. It is shown in [49] that replacing $a_{1} u$ by $a_{1} u^{\kappa}$ with suitable $\kappa<1$ (for instance, $\kappa=1 / 2$ ) and replacing $u-v$ by $u-\frac{1}{|\Omega|} \int_{\Omega} u(x) d x$, then finite-time blow-up is possible.

However, as far as $\chi \neq 0$ and $a_{0}(t, x), a_{i}(t, x), a_{2}(t, x)$ are not constants, there is little study of (1.3). The objective of the present paper is to investigate the asymptotic dynamics of (1.3). To this end, we first study the local and global existence of classical solutions of (1.3) with given nonnegative initial functions, next study the existence of entire positive solutions, and then investigate the uniqueness and stability of entire positive solutions and the asymptotic behavior of positive solutions of (1.3). Throughout this paper, we assume that $a_{i}(i=0,1,2)$ satisfy the following standing assumption.

(H1) $a_{0}(t, x), a_{1}(t, x)$ and $a_{2}(t, x)$ are Hölder continuous in $t \in \mathbb{R}$ with exponent $\nu>0$ uniformly with respect to $x \in \bar{\Omega}$, continuous in $x \in \bar{\Omega}$ uniformly with respect to $t \in \mathbb{R}$, and there are nonnegative constants $\alpha_{i}, A_{i}(i=0,1,2)$ with $\alpha_{1}+\alpha_{2}>0$ such that

$$
\left\{\begin{array}{l}
0<\alpha_{0} \leq a_{0}(t, x) \leq A_{0} \\
0 \leq \alpha_{1} \leq a_{1}(t, x) \leq A_{1} \\
0 \leq \alpha_{2} \leq\left|a_{2}(t, x)\right| \leq A_{2}
\end{array}\right.
$$

A vector valued function $(u(x, t), v(x, t))$ is called a classical solution of (1.3) on $\Omega \times\left(t_{1}, t_{2}\right)$ $\left(-\infty \leq t_{1}<t_{2} \leq \infty\right)$ if $(u, v) \in C\left(\bar{\Omega} \times\left(t_{1}, t_{2}\right)\right) \cap C^{2,1}\left(\bar{\Omega} \times\left(t_{1}, t_{2}\right)\right)$ and satisfies (1.3) for $t \in\left(t_{1}, t_{2}\right)$ in the classical sense. A classical solution $(u(x, t), v(x, t))$ of (1.3) on $\Omega \times\left(t_{1}, t_{2}\right)$ is called nonnegative if $u(x, t) \geq 0$ and $v(x, t) \geq 0$ for $(x, t) \in \bar{\Omega} \times\left(t_{1}, t_{2}\right)$, and is called positive if $\inf _{(x, t) \in \bar{\Omega} \times\left(t_{1}, t_{2}\right)} u(x, t)>0$ and $\inf _{(x, t) \in \bar{\Omega} \times\left(t_{1}, t_{2}\right)} v(x, t)>0 . \quad(u(x, t), v(x, t))$ is called an entire classical solution of (1.3) if it is a classical solution of (1.3) on $(-\infty, \infty)$. For a given $t_{0} \in \mathbb{R}$ and a given function $u_{0}(\cdot)$ on $\Omega$, it is said that (1.3) has a classical solution with initial condition $u\left(x, t_{0}\right)=u_{0}(x)$ if (1.3) has a classical solution, denoted by $\left(u\left(x, t ; t_{0}, u_{0}\right), v\left(x, t ; t_{0}, u_{0}\right)\right)$, on $\left(t_{0}, T\right)$ for some $T>t_{0}$ satisfying that $\lim _{t \rightarrow t_{0}+} u\left(\cdot, t ; t_{0}, u_{0}\right)=u_{0}(\cdot)$ in certain sense. A classical solution of (1.3) with initial condition $u\left(x, t_{0}\right)=u_{0}(x)$ exists globally if (1.3) has a classical solution $\left(u\left(x, t ; t_{0}, u_{0}\right), v\left(x, t ; t_{0}, u_{0}\right)\right)$ with $u\left(x, t_{0} ; t_{0}, u_{0}\right)=u_{0}(x)$ on $\left(t_{0}, \infty\right)$. 
For given $1 \leq p<\infty$, let $X=L^{p}(\Omega)$ and $A=-\Delta+I$ with

$$
D(A)=\left\{u \in W^{2, p}(\Omega) \mid \frac{\partial u}{\partial n}=0 \quad \text { on } \partial \Omega\right\} .
$$

It is well known that $A$ is a sectorial operator in $X$ (see, for example, [11, Example 1.6]) and thus generates an analytic semigroup $\left(e^{-A t}\right)_{t \geq 0}$ in $X$ (see, for example, [11, Theorem 1.3.4]). Moreover $0 \in \rho(A)$ and

$$
\left\|e^{-A t} u\right\|_{X} \leq e^{-t}\|u\|_{X} \quad \text { for } \quad t \geq 0 \text { and } u \in X
$$

Because $\Delta$ is dissipative operator and $\operatorname{range}(I-\Delta)=X$, so it generates a strongly continuous semigroup of contraction on $X$.

Let $X^{\alpha}=D\left(A^{\alpha}\right)$ equipped with the graph norm $\|u\|_{\alpha}:=\|u\|_{X^{\alpha}}=\left\|A^{\alpha} u\right\|_{p}$ (see, for example, [11, Definition 1.4.7]).

Throughout this paper, $A$ and $X^{\alpha}$ are defined as in the above. For given $-\infty \leq t_{1}<t_{2} \leq \infty$ and $0 \leq \delta<1, C^{\delta}\left(\left(t_{1}, t_{2}\right), X^{\alpha}\right)$ is the space of all locally Hölder continuous functions from $\left(t_{1}, t_{2}\right)$ to $X^{\alpha}$ with exponent $\delta$. First of all, we have the following local existence theorem.

Theorem 1.1. Suppose that $p>1$ and $1 / 2<\alpha<1$ are such that $X^{\alpha} \subset C^{1}(\bar{\Omega})$.

(1) For any $t_{0} \in \mathbb{R}$ and $u_{0} \in X^{\alpha}$ with $u_{0} \geq 0$, there exists $T_{\max } \in(0, \infty]$ such that (1.3) has a unique non-negative classical solution $\left(u\left(x, t ; t_{0}, u_{0}\right), v\left(x, t ; t_{0}, u_{0}\right)\right)$ on $\left(t_{0}, t_{0}+T_{\max }\right)$ satisfying that $\lim _{t \rightarrow t_{0}}\left\|u\left(\cdot, t ; t_{0}, u_{0}\right)-u_{0}(\cdot)\right\|_{X^{\alpha}}=0$, and

$$
u\left(\cdot, \cdot ; t_{0}, u_{0}\right) \in C\left(\left[t_{0}, t_{0}+T_{\max }\right), X^{\alpha}\right) \cap C^{\delta}\left(\left(t_{0}, t_{0}+T_{\max }\right), X^{\alpha}\right)
$$

for some $0<\delta<1$. Moreover if $T_{\max }<\infty$, then

$$
\limsup _{t \nearrow T_{\max }}\left\|u\left(\cdot, t+t_{0} ; t_{0}, u_{0}\right)\right\|_{X^{\alpha}}=\limsup _{t \nearrow T_{\max }}\left\|u\left(\cdot, t+t_{0} ; t_{0}, u_{0}\right)\right\|_{C^{0}(\bar{\Omega})}=\infty
$$

(2) For any given $t_{0} \in \mathbb{R}$ and $u_{0} \in C^{0}(\bar{\Omega})$ with $u_{0} \geq 0$, there exists $T_{\max } \in(0, \infty]$ such that (1.3) has a unique non-negative classical solution $\left(u\left(x, t ; t_{0}, u_{0}\right), v\left(x, t ; t_{0}, u_{0}\right)\right)$ on $\left(t_{0}, t_{0}+T_{\max }\right)$ satisfying that $\lim _{t \rightarrow t_{0}}\left\|u\left(\cdot, t ; t_{0}, u_{0}\right)-u_{0}(\cdot)\right\|_{C^{0}(\bar{\Omega})}=0$, and

$$
u\left(\cdot, \cdot ; t_{0}, u_{0}\right) \in C\left(\left(t_{0}, t_{0}+T_{\max }\right), X^{\alpha}\right) \cap C^{\delta}\left(\left(t_{0}, t_{0}+T_{\max }\right), X^{\alpha}\right)
$$

for some $0<\delta<1$. Moreover if $T_{\max }<\infty$, then

$$
\limsup _{t \nearrow T_{\max }}\left\|u\left(\cdot, t+t_{0} ; t_{0}, u_{0}\right)\right\|_{C^{0}(\bar{\Omega})}=\infty
$$

Remark 1.1. (1) Since $X^{\alpha} \subset C^{1}(\bar{\Omega}) \subset C^{0}(\bar{\Omega})$, the existence of a local classical solution in Theorem 1.1(1) is guaranteed by Theorem 1.1(2). However $\lim _{t \rightarrow t_{0}} u\left(\cdot, \cdot ; t_{0}, u_{0}\right)=u_{0}(\cdot)$ in the $X^{\alpha}$-norm in Theorem 1.1](1) is not included in Theorem 1.1 (2).

(2) Theorem 1.1(2) is consistent (one species version) with [39, Lemma 2.1]. 
(3) Semigroup theory and fixed point theorems together with regularity and a prior estimates for elliptic and parabolic equations are among basic tools used in literature to prove the local existence of classical solutions of chemotaxis models with various given initial functions. For the self-completeness, we will give a proof of Theorem 1.1(1) by using semigroup theory and give a proof of Theorem 1.1(2) based on the combination of fixed point theorems and semigroup theory.

We next consider the global existence of classical solutions of (1.3) with given initial functions. Throughout the paper, we put

$$
\begin{gathered}
a_{i, \inf }=\inf _{t \in \mathbb{R}, x \in \bar{\Omega}} a_{i}(t, x), \quad a_{i, \sup }=\sup _{t \in \mathbb{R}, x \in \bar{\Omega}} a_{i}(t, x), \\
a_{i, \inf }(t)=\inf _{x \in \bar{\Omega}} a_{i}(t, x), \quad a_{i, \sup }(t)=\sup _{x \in \bar{\Omega}} a_{i}(t, x),
\end{gathered}
$$

unless specified otherwise. For convenience, we introduce the following assumptions.

(H2) $a_{1}(t, x), a_{2}(t, x)$, and $\chi$ satisfy

$$
\inf _{t \in \mathbb{R}}\left\{a_{1, \inf }(t)-|\Omega|\left(a_{2, \inf }(t)\right)_{-}\right\}>(\chi)_{+},
$$

where $|\Omega|$ is the Lebesgue measure of $\Omega$ and $(\chi)_{+}=\max \{0, \chi\}$.

(H2)' $a_{1}(t, x), a_{2}(t, x)$, and $\chi$ satisfy $\inf _{t \in \mathbb{R}}\left\{a_{1, \inf }(t)-|\Omega|\left(a_{2, \inf }(t)\right)_{-}\right\}>0$ and if $n \geq 3$, $a_{1, \inf }>\frac{\chi(n-2)}{n}$.

The following is our main result on the global existence of positive classical solutions to system (1.3).

Theorem 1.2. (1) Assume that (H2) holds. Then for any $t_{0} \in \mathbb{R}$ and $u_{0} \in C^{0}(\bar{\Omega})$ with $u_{0} \geq$ 0 , (1.3) has a unique global classical solution $\left(u\left(x, t ; t_{0}, u_{0}\right), v\left(x, t ; t_{0}, u_{0}\right)\right)$ which satisfies that $\lim _{t \rightarrow t_{0}}\left\|u\left(\cdot, t ; t_{0}, u_{0}\right)-u_{0}(\cdot)\right\|_{C^{0}(\bar{\Omega})}=0$ and (1.8), (1.9). Moreover, we have

$$
\begin{aligned}
0 \leq v\left(x, t ; t_{0}, u_{0}\right) & \leq \max _{x \in \bar{\Omega}} u\left(x, t ; t_{0}, u_{0}\right) \\
& \leq \max \left\{\sup u_{0}(x), \frac{a_{0, \text { sup }}}{\inf _{t \geq t_{0}}\left\{a_{1, \text { inf }}(t)-|\Omega|\left(a_{2, \text { inf }}(t)\right)_{-}-(\chi)_{+}\right\}}\right\}
\end{aligned}
$$

for all $(x, t) \in \bar{\Omega} \times\left[t_{0}, \infty\right)$.

(2) Assume that (H2)' holds. Then for any $t_{0} \in \mathbb{R}$ and $u_{0} \in C^{0}(\bar{\Omega})$ with $u_{0} \geq 0$, system (1.3) has a unique global classical solution $\left(u\left(x, t ; t_{0}, u_{0}\right), v\left(x, t ; t_{0}, u_{0}\right)\right)$ which satisfies that $\lim _{t \rightarrow t_{0}}\left\|u\left(\cdot, t ; t_{0}, u_{0}\right)-u_{0}(\cdot)\right\|_{C^{0}(\bar{\Omega})}=0$ and (1.8), (1.9). Moreover,

$$
\left\|u\left(\cdot, t ; t_{0}, u_{0}\right)\right\|_{C^{0}(\bar{\Omega})}+\left\|v\left(\cdot, t ; t_{0}, u_{0}\right)\right\|_{C^{0}(\bar{\Omega})} \leq C
$$

for all $t \geq t_{0}$, where $C=C\left(\left\|u_{0}\right\|_{C^{0}(\bar{\Omega})}\right)$, i.e, $C$ depends only on $\left\|u_{0}\right\|_{C^{0}(\bar{\Omega})}$, and

$$
0 \leq \int_{\Omega} u\left(x, t ; t_{0}, u_{0}\right) d x \leq \max \left\{\int_{\Omega} u_{0}(x), \frac{|\Omega| a_{0, \text { sup }}}{\inf _{t \in \mathbb{R}}\left\{a_{1, \text { inf }}(t)-|\Omega|\left(a_{2, \text { inf }}(t)\right)_{-}\right\}}\right\} \forall t \geq t_{0} .
$$


Remark 1.2. (1) When $a_{2}(t, x) \geq 0$, (H2)' becomes $a_{1, \inf }>\max \left\{\frac{\chi(n-2)}{n}, 0\right\}$. In particular, if $a_{2}(t, x)=0$, Theorem 1.2 is consistent with the result by Tello and Winkler in 43].

(2) When the coefficients are constant, the condition (H2) becomes $a_{1}-|\Omega|\left(a_{2}\right)_{-}>(\chi)_{+}$ which is consistent with the result of global existence by Negreanu and Tello in [31].

(3) (H2) implies (H2)'. Therefore the global existence of bounded classical solutions of (1.3) in Theorem 1.2(1) follows from Theorem 1.2(2). However the explicit bound given by (1.13) is not included in Theorem 1.2(2). Note that the explicit bound (1.13) will be used in the proof of the existence of periodic solutions (resp. steady state solutions) when the coefficients $a_{i}(t, x)$ are periodic (resp. when $a_{i}(t, x)=a_{i}(x)$ ) (see Theorem 1.3).

(4) In general, assuming that $\inf _{t \in \mathbb{R}}\left\{a_{1, \mathrm{inf}}(t)-|\Omega|\left(a_{2, \mathrm{inf}}(t)\right)_{-}\right\}>0$, it remains open whether for any given $t_{0} \in \mathbb{R}$ and $u_{0} \in C^{0}(\bar{\Omega})$, (1.3) has a global classical solution $\left(u\left(x, t ; t_{0}, u_{0}\right)\right.$, $\left.v\left(x, t ; t_{0}, u_{0}\right)\right)$. This is open even in the case that $a_{i}(t, x) \equiv a_{i}$ for $i=0,1$ and $a_{2}(t, x)=0$.

(5) When $\chi \leq 0$, conditions (H2) and (H2)' become $\inf _{t \in \mathbb{R}}\left\{a_{1, \inf }(t)-|\Omega|\left(a_{2, \text { inf }}(t)\right)_{-}\right\}>0$. Thus global existence holds under this weak condition.

We now state our main result on the existence of entire bounded positive solutions of (1.3).

Theorem 1.3. Suppose that (H2) holds. Then there is an entire positive bounded classical solution $(u, v)=\left(u^{*}(x, t), v^{*}(x, t)\right)$ of (1.3). Moreover, the following hold.

(1) If there is $T>0$ such that $a_{i}(t+T, x)=a_{i}(t, x)$ for $i=0,1,2$, then (1.3) has a positive periodic solution $(u, v)=\left(u^{*}(x, t), v^{*}(x, t)\right)$ with period $T$.

(2) If $a_{i}(t, x) \equiv a_{i}(t)$ for $i=0,1,2$, then (1.3) has a unique entire positive spatially homogeneous solution $(u, v)=\left(u^{*}(t), v^{*}(t)\right)$ with $v^{*}(t)=u^{*}(t)$, and if $a_{i}(t)(i=0,1,2)$ are periodic or almost periodic, so is $\left(u^{*}(t), v^{*}(t)\right)$.

(3) If $a_{i}(t, x) \equiv a_{i}(x)$ for $i=0,1,2$, then (1.3) has a positive steady state solution $(u, v)=$ $\left(u^{*}(x), v^{*}(x)\right)$.

Remark 1.3. (1) When the coefficients are only time dependent, i.e, $a_{i}(t, x)=a_{i}(t)$ for $i=$ $0,1,2$, every entire positive solution $u(t)$ of the $O D E$

$$
u_{t}=u\left[a_{0}(t)-\left(a_{1}(t)+|\Omega| a_{2}(t)\right) u\right]
$$

is an entire positive solution of the first equation of (1.3) and then $(u(t), v(t))$ with $v(t)=$ $u(t)$ is an entire positive solution of (1.3). Thus (1.3) has an entire solution under the weaker assumption $\inf _{t \in \mathbb{R}}\left\{a_{1}(t)-|\Omega|\left(a_{2}(t)\right)_{-}\right\}>0$ (see Lemma 2.5). In general, due to the lack of comparison principle for system (1.3), it is fairly nontrivial to prove the existence of entire positive solutions.

(2) It should be mentioned that there may be lots of entire positive solutions (see [26], [43]).

(3) The existence of entire positive bounded classical of (1.3) also holds under the weaker assumption (H2)' (see Remarks 4.1 and 5.1). However under $\mathbf{( H 2 )})^{\prime}$, it reminds open whether there are periodic solutions of (1.3) when the coefficients $a_{i}(t, x)$ are periodic (resp. steady state solutions of (1.3) when $a_{i}(t, x) \equiv a_{i}(x)$ ). 
Finally we state the main results on the stability and uniqueness of entire positive solutions and asymptotic behavior of positive solutions of (1.3).

Theorem 1.4. (1) If $a_{i}(t, x) \equiv a_{i}(t)$ for $i=0,1,2$ and

$$
\inf _{t}\left\{a_{1}(t)-|\Omega|\left|a_{2}(t)\right|\right\}>2(\chi)_{+},
$$

then for any $t_{0} \in \mathbb{R}$ and $u_{0} \in C^{0}(\bar{\Omega})$ with $u_{0} \geq 0$ and $u_{0} \not \equiv 0$, the unique global classical solution $\left(u\left(x, t ; t_{0}, u_{0}\right), v\left(x, t ; t_{0}, u_{0}\right)\right)$ of (1.3) satisfies

$$
\lim _{t \rightarrow \infty}\left(\left\|u\left(\cdot, t ; t_{0}, u_{0}\right)-u^{*}(t)\right\|_{C^{0}(\bar{\Omega})}+\left\|v\left(\cdot, t ; t_{0}, u_{0}\right)-u^{*}(t)\right\|_{C^{0}(\bar{\Omega})}\right)=0,
$$

where $u^{*}(t)$ is the unique spatially homogeneous entire positive solution of (1.3).

(2) Suppose that

$$
\inf _{t \in \mathbb{R}}\left\{a_{1, \inf }(t)-|\Omega|\left(a_{2, \inf }(t)\right)_{-}\right\}>\left\{(\chi)_{+}+\frac{a_{0, \sup }}{a_{0, \text { inf }}}\left((\chi)_{+}+|\Omega| \sup _{t \in \mathbb{R}}\left(a_{2, \sup }(t)\right)_{+}\right)\right\}
$$

and

$$
\limsup _{t-s \rightarrow \infty} \frac{1}{t-s} \int_{s}^{t}\left(L_{2}(\tau)-L_{1}(\tau)\right) d \tau<0
$$

where

$$
\begin{gathered}
L_{1}(t)=2 r_{2}\left(a_{1, \text { inf }}(t)+|\Omega|\left(a_{2, \text { inf }}(t)\right)_{+}\right), \\
L_{2}(t)=a_{0, \text { sup }}(t)+\frac{\chi}{2}\left(r_{1}-r_{2}\right)+\frac{\left(\chi r_{1}\right)^{2}}{2}+|\Omega| r_{1}\left(2\left(a_{2, \text { inf }}(t)\right)_{-}+\left(a_{2, \text { sup }}(t)\right)_{+}\right),
\end{gathered}
$$

and

$$
\begin{aligned}
& r_{1}=\frac{\sup _{t \in \mathbb{R}}\left\{a_{1, \sup }(t)-|\Omega|\left(a_{2, \sup }(t)\right)_{-}-(\chi)_{+}\right\} a_{0, \sup }-a_{0, \inf }\left((\chi)_{+}+|\Omega| \inf _{t}\left(a_{2, \inf }(t)\right)_{+}\right)}{h(\chi)}, \\
& r_{2}=\frac{\inf _{t \in \mathbb{R}}\left\{a_{1, \text { inf }}(t)-|\Omega|\left(a_{2, \text { inf }}(t)\right)_{-}-(\chi)_{+}\right\} a_{0, \text { inf }}-a_{0, \text { sup }}\left((\chi)_{+}+|\Omega| \sup _{t}\left(a_{2, \text { sup }}(t)\right)_{+}\right)}{h(\chi)} \\
& h(\chi)=\inf _{t \in \mathbb{R}}\left\{a_{1, \inf }(t)-|\Omega|\left(a_{2, \inf }(t)\right)_{-}-(\chi)_{+}\right\} \sup _{t \in \mathbb{R}}\left\{a_{1, \sup }(t)-|\Omega|\left(a_{2, \sup }(t)\right)_{-}-(\chi)_{+}\right\} \\
& -\left((\chi)_{+}+|\Omega| \inf _{t \in \mathbb{R}}\left(a_{2, \inf }(t)\right)_{+}\right)\left((\chi)_{+}+|\Omega| \sup _{t \in \mathbb{R}}\left(a_{2, \text { sup }}(t)\right)_{+}\right) .
\end{aligned}
$$

Then (1.3) has a unique entire positive solution $\left(u^{*}(x, t), v^{*}(x, t)\right)$, and, for any $t_{0} \in \mathbb{R}$ and $u_{0} \in C^{0}(\bar{\Omega})$ with $u_{0} \geq 0$ and $u_{0} \not \equiv 0$, the global classical solution $\left(u\left(x, t ; t_{0}, u_{0}\right)\right.$, $\left.v\left(x, t ; t_{0}, u_{0}\right)\right)$ of (1.3) satisfies

$$
\lim _{t \rightarrow \infty}\left(\left\|u\left(\cdot, t ; t_{0}, u_{0}\right)-u^{*}(\cdot, t)\right\|_{C^{0}(\bar{\Omega})}+\left\|v\left(\cdot, t ; t_{0}, u_{0}\right)-v^{*}(\cdot, t)\right\|_{C^{0}(\bar{\Omega})}\right)=0 .
$$

If, in addition, $a_{i}(t, x) \equiv a_{i}(x)$ (resp. $a_{i}(t+T, x)=a_{i}(t, x), a_{i}(t, x)$ is almost periodic in $t$ uniformly with respect to $x$ ) for $i=0,1,2$, then (1.3) has a unique positive steady state solution $\left(u^{*}(x), v^{*}(x)\right)$ (resp. (1.3) has a unique time periodic positive solution $\left(u^{*}(x, t), v^{*}(x, t)\right)$ with period $T$, (1.3) has a unique time almost periodic solution $\left.\left(u^{*}(x, t), v^{*}(x, t)\right)\right)$. 
Theorem 1.5. Suppose that (1.16) holds and $r_{1}$ and $r_{2}$ are as in Theorem 1.4(2). Then

(1) For any $t_{0} \in \mathbb{R}, u_{0} \in C^{0}(\bar{\Omega})$ with $u_{0} \geq 0$ and $u_{0} \not \equiv 0$, and $\epsilon>0$, there exists $t_{\epsilon}$ such that

$$
r_{2}-\epsilon \leq u\left(x, t ; t_{0}, u_{0}\right) \leq r_{1}+\epsilon, r_{2}-\epsilon \leq v\left(x, t ; t_{0}, u_{0}\right) \leq r_{1}+\epsilon
$$

for all $x \in \bar{\Omega}$ and $t \geq t_{0}+t_{\epsilon}$.

(2) Moreover if the coefficients $a_{i}$ are periodic in $t$ with period $T>0$ (resp. $a_{i}$ are almost periodic in $t$ ), then there are $T$-periodic functions $m(t)$ and $M(t)$ (resp. almost periodic functions $m(t)$ and $M(t))$ with

$$
r_{2} \leq \inf _{t \in \mathbb{R}} m(t) \leq m(t) \leq M(t) \leq \sup _{t \in \mathbb{R}} M(t) \leq r_{1}
$$

such that for any $t_{0} \in \mathbb{R}, u_{0} \in C(\bar{\Omega})$ with $u_{0} \geq 0$ and $u_{0} \not \equiv 0$, and $\epsilon>0$, there is $t_{\epsilon}>0$ such that

$$
m(t)-\epsilon \leq u\left(x, t ; t_{0}, u_{0}\right) \leq M(t)+\epsilon, \quad m(t)-\epsilon \leq v\left(x, t ; t_{0}, u_{0}\right) \leq M(t)+\epsilon \quad \forall x \in \bar{\Omega}, t \geq t_{0}+t_{\epsilon} .
$$

Remark 1.4. (1) When $a_{i}(i=0,1,2)$ are constants, the condition (1.14) becomes

$$
a_{1}-|\Omega|\left|a_{2}\right|>2(\chi)_{+} .
$$

Theorem 1.4(1) is then an extension of [31, Theorem 0.1] by Negreanu and Tello. When the nonlocal term is zero, the result in Theorem 1.4(1) is consistent with the result by Tello and Winkler in 43].

(2) In Theorem 1.4(2), when $a_{i}(i=0,1,2)$ are constants, we have $r_{1}=r_{2}=\frac{a_{0}}{a_{1}+|\Omega| a_{2}}$ and

$$
L_{1}(t)=\frac{2 a_{0}\left(a_{1}+|\Omega|\left(a_{2}\right)_{+}\right)}{a_{1}+|\Omega| a_{2}}, \quad L_{2}(t)=a_{0}+\frac{\chi^{2} a_{0}^{2}}{2\left(a_{1}+|\Omega| a_{2}\right)^{2}}+\frac{a_{0}|\Omega|\left[2\left(a_{2}\right)_{-}+\left(a_{2}\right)_{+}\right]}{a_{1}+|\Omega| a_{2}} .
$$

Hence the condition (1.16) becomes (1.23) and the condition (1.17) becomes

$$
\frac{\chi^{2} a_{0}}{2\left(a_{1}+|\Omega| a_{2}\right)}<a_{1}-|\Omega|\left(a_{2}\right)_{-} .
$$

Furthermore when $\chi=0$, the condition (1.17) becomes

$$
a_{1}-|\Omega|\left(a_{2}\right)_{-}>0 \text {. }
$$

(3) In Theorem 1.4(2), if $a_{i}(t+T, x)=a_{i}(t, x)(i=0,1,2)$, then (1.17) becomes

$$
\int_{0}^{T}\left(L_{2}(t)-L_{1}(t)\right) d t<0 .
$$

(4) It is seen from Theorem 1.3 that (1.12) ensures the existence of entire positive solutions of (1.3). In the case that $a_{i}(t, x) \equiv a_{i}(t)(i=0,1,2)$, the condition (1.14) ensures the stability and uniqueness of entire positive solutions of (1.3). In the general case, Theorem 1.5 provides some positive attracting set for positive solutions of (1.3) under the condition (1.16). It remains open whether in the general case, the condition (1.16) also ensures the stability and uniqueness of entire positive solutions of (1.3). 
(5) The reader is referred to Definition 2.3 for the definition of almost periodic functions.

The rest of the paper is organized as follows. In section 2, we collect some important results from literature that will be used in the proofs of our main results. In section 3, we study the local existence of classical solutions of (1.3) with given initial functions and prove Theorem 1.1. In section 4, we investigate the global existence of classical solutions of (1.3) with given initial functions and prove Theorem 1.2. We consider the existence of entire positive solutions of (1.3) and prove Theorem 1.3 in section 5 . Finally, in section 6 , we study the asymptotic behavior of global positive solutions and prove Theorems 1.4 and 1.5 .

Acknowledgment. The authors would like to thank Professors J. Ignacio Tello and Michael Winkler for valuable discussions, suggestions, and references.

\section{Preliminaries}

In this section, we recall some standard definitions and lemmas from semigroup theory. We also present some known results on non-autonomous logistic equations and Lotka-Volterra competition systems.

\subsection{Semigroup theory}

In this subsection, we recall some standard definitions and lemmas from semigroup theory. The reader is referred to [11, 34] for the details.

Recall that for given $1 \leq p<\infty, A=-\Delta+I$ with

$$
D(A)=\left\{u \in W^{2, p}(\Omega) \mid \frac{\partial u}{\partial n}=0 \quad \text { on } \partial \Omega\right\}
$$

and $X^{\alpha}=D\left(A^{\alpha}\right)$ equipped with the graph norm $\|x\|_{\alpha}=\left\|A^{\alpha} x\right\|_{p}$. Note that $X^{0}=L^{p}(\Omega)$.

Lemma 2.1. (See [11, Theorem 1.6.1]) Let $1 \leq p<\infty$. For any $0 \leq \alpha \leq 1$, we have

$$
X^{\alpha} \subset C^{\nu}(\bar{\Omega}) \text { when } 0 \leq \nu<2 \alpha-\frac{n}{p}
$$

where the inclusion is continuous. In particular when $\frac{n}{2 p}<\alpha \leq 1$, we get $X^{\alpha} \subset C^{0}(\bar{\Omega})$.

Lemma 2.2. (See [19, Lemma 2.1]) Let $\beta \geq 0$ and $p \in(1, \infty)$. Then for any $\epsilon>0$ there exists $C(\epsilon)>0$ such that for any $w \in C_{0}^{\infty}(\Omega)$ we have

$$
\left\|A^{\beta} e^{-t A} \nabla \cdot w\right\|_{L^{p}(\Omega)} \leq C(\epsilon) t^{-\beta-\frac{1}{2}-\epsilon} e^{-\mu t}\|w\|_{L^{p}(\Omega)} \quad \text { for all } t>0 \text { and some } \mu>0 .
$$

Accordingly, for all $t>0$ the operator $A^{\beta} e^{-t A} \nabla \cdot$ admits a unique extension to all of $L^{p}(\Omega)$ which is again denoted by $A^{\beta} e^{-t A} \nabla \cdot$ and satisfies (2.1) for all $w \in L^{p}(\Omega)$.

Consider

$$
\left\{\begin{array}{l}
u_{t}+A u=F(t, u), t>t_{0} \\
u\left(t_{0}\right)=u_{0} .
\end{array}\right.
$$

We assume that $F$ maps some open set $U$ of $\mathbb{R} \times X^{\alpha}$ into $X^{0}$ for some $0 \leq \alpha<1$, and $F$ is locally Hölder continuous in $t$ and locally Lipschitz continuous in $u$ for $(t, u) \in U$. 
Definition 2.1 (Mild solution). For given $u_{0} \in X^{\alpha}$. A continuous function $u:\left[t_{0}, t_{1}\right) \rightarrow X^{0}$ is called a mild solution of (2.2) on $t_{0}<t<t_{1}$ if $u(t) \in X^{\alpha}$ for $t \in\left[t_{0}, t_{1}\right)$ and the following integral equation holds on $t_{0}<t<t_{1}$,

$$
u(t)=e^{-A\left(t-t_{0}\right)} u_{0}+\int_{t_{0}}^{t} e^{-A(t-s)} F(s, u(s)) d s .
$$

Definition 2.2 (Strong solution). (see [11, Definition 3.3.1]) A strong solution of the Cauchy problem (2.2) on $\left(t_{0}, t_{1}\right)$ is a continuous function $u:\left[t_{0}, t_{1}\right) \rightarrow X^{0}$ such that $u\left(t_{0}\right)=u_{0}, u(t) \in$ $D(A)$ for $t \in\left(t_{0}, t_{1}\right)$, $\frac{d u}{d t}$ exists for $t \in\left(t_{0}, t_{1}\right),\left(t_{0}, t_{1}\right) \ni t \rightarrow F(t, u(t)) \in X^{0}$ is locally Hölder continuous, and $\int_{t_{0}}^{t_{0}+\sigma}\|F(t, u(t))\| d t<\infty$ for some $\sigma>0$, and the differential equation $u_{t}+A u=$ $F(t, u)$ is satisfied on $\left(t_{0}, t_{1}\right)$.

Lemma 2.3 (Existence of mild/strong solutions). (1) For any $\left(t_{0}, u_{0}\right) \in U$ there exists $T_{\max }=$ $T_{\max }\left(t_{0}, u_{0}\right)>0$ such that (2.2) has a unique strong solution $u\left(t ; t_{0}, u_{0}\right)$ on $\left(t_{0}, t_{0}+T_{\max }\right)$ with initial value $u\left(t_{0} ; t_{0}, u_{0}\right)=u_{0}$. Moreover, $u\left(\cdot ; t_{0}, u_{0}\right) \in C\left(\left[t_{0}, t_{0}+T_{\max }\right), X^{\alpha}\right)$ and if $T_{\max }<\infty$, then

$$
\limsup _{t \nearrow T_{\max }}\left\|u\left(t+t_{0} ; t_{0}, u_{0}\right)\right\|_{X^{\alpha}}=\infty .
$$

(2) For given $\left(t_{0}, u_{0}\right) \in U$, if $u(t)$ is a strong solution of (2.2) on $\left(t_{0}, t_{1}\right)$, then $u$ satisfy the integral equation (2.3). Conversely, if $u(t)$ is continuous function from $\left(t_{0}, t_{1}\right)$ into $X^{\alpha}$, $\int_{t_{0}}^{t_{0}+\sigma}\|F(t, u(t))\| d t<\infty$ for some $\sigma>0$, and if the integral equation (2.3) holds for $t_{0}<t<t_{1}$, then $u(t)$ is a strong solution of the differential equation (2.2) on $\left(t_{0}, t_{1}\right)$. Furthermore,

$$
u \in C^{\delta}\left(\left(t_{0}, t_{1}\right), X^{\alpha}\right) \text { for all } \delta \text { such that } 0<\delta<1-\alpha
$$

Proof. (1) It follows from [11, Theorem 3.3.3 ] and [11, Theorem 3.3.4 ].

(2) The equivalence part follows from [11, Lemma 3.3.2] and $u \in C^{\delta}\left(\left(t_{0}, t_{1}\right) ; X^{\alpha}\right)$ follows from the proof of [11, Lemma 3.3.2].

\subsection{Nonautonomous logistic equations and Lotka-Volterra competition sys- tems}

In this subsection, we first recall the definition of almost periodic functions and some basic properties of almost periodic functions. We then review some known results for nonautonomous logistic equations and Lotka-Volterra competition systems.

Definition 2.3. (1) A continuous function $f: \mathbb{R} \rightarrow \mathbb{C}$ is Bohr almost periodic if for any $\epsilon>0$, the set of $\epsilon$-periods $\{\tau|| f(t+\tau)-f(t) \mid<\epsilon\}$ is relatively dense in $\mathbb{R}$, i.e, there exists an $l=l(\epsilon)$ such that every interval of the form $[t, t+l]$ intersects the set of $\epsilon$-periods.

(2) Let $g(t, x)$ be a continuous function of $(t, x) \in \mathbb{R} \times \bar{\Omega} . g$ is said to be almost periodic in $t$ uniformly with respect to $x \in \bar{\Omega}$ if $g$ is uniformly continuous in $t \in \mathbb{R}$ and $x \in \bar{\Omega}$, and for each $x \in \bar{\Omega}, g(t, x)$ is almost periodic in $t$. 
Lemma 2.4. Let $g(t, x)$ be a continuous function of $(t, x) \in \mathbb{R} \times \bar{\Omega} . g$ is almost periodic in $t$ uniformly with respect to $x \in \bar{\Omega}$ if and only if $g$ is uniformly continuous in $t \in \mathbb{R}$ and $x \in \bar{\Omega}$, and for any sequences $\left\{\beta_{n}^{\prime}\right\},\left\{\gamma_{n}^{\prime}\right\} \subset \mathbb{R}$, there are subsequences $\left\{\beta_{n}\right\} \subset\left\{\beta_{n}^{\prime}\right\},\left\{\gamma_{n}\right\} \subset\left\{\gamma_{n}^{\prime}\right\}$ such that

$$
\lim _{n \rightarrow \infty} \lim _{m \rightarrow \infty} g\left(t+\beta_{n}+\gamma_{m}, x\right)=\lim _{n \rightarrow \infty} g\left(t+\beta_{n}+\gamma_{n}, x\right) \quad \forall(t, x) \in \mathbb{R} \times \bar{\Omega} .
$$

Proof. See [6, Theorems 1.17 and 2.10].

Consider the following nonautonomous logistic equations

$$
\frac{d u}{d t}=u(a(t)-b(t) u)
$$

where $a(t)$ and $b(t)$ are continuous functions. For given $u_{0} \in \mathbb{R}$, let $u\left(t ; t_{0}, u_{0}\right)$ be the solution of (2.4) with $u\left(t_{0} ; t_{0}, u_{0}\right)=u_{0}$.

Lemma 2.5. (see [33], Theorems 2.1, 3.1 and 4.1) Suppose that $a(t)$ and $b(t)$ are continuous and satisfy that $0<\inf _{t \in \mathbb{R}} a(t) \leq \sup _{t \in \mathbb{R}} a(t)<\infty, \quad 0<\inf _{t \in \mathbb{R}} b(t) \leq \sup _{t \in \mathbb{R}} b(t)<\infty$. Then

(1) The non-autonomous equation (2.4) has exactly one bounded entire solution $u^{*}(t)$ that is positive and satisfies

$$
\frac{\inf _{t \in \mathbb{R}} a(t)}{\sup _{t \in \mathbb{R}} b(t)} \leq u^{*}(t) \leq \frac{\sup _{t \in \mathbb{R}} a(t)}{\inf _{t \in \mathbb{R}} b(t)} \quad \forall t \in \mathbb{R}
$$

(2) $u^{*}(\cdot)$ is an attractor for all positive solutions of (2.4), that is, for any $u_{0}>0$ and $t_{0} \in \mathbb{R}$,

$$
\lim _{t \rightarrow \infty}\left\|u\left(t+t_{0} ; t_{0}, u_{0}\right)-u^{*}\left(t+t_{0}\right)\right\|=0 .
$$

(3) If furthermore $a(t)$ and $b(t)$ are periodic with period $T$ (resp. almost periodic), $u^{*}(t)$ is also periodic with period $T$ (resp. almost periodic).

Consider now the following nonautonomous Lotka-Volterra competition systems

$$
\left\{\begin{array}{l}
\frac{d u}{d t}=u\left(a_{1}(t)-b_{1}(t) u-c_{1}(t) v\right) \\
\frac{d v}{d t}=v\left(a_{2}(t)-b_{2}(t) u-c_{2}(t) v\right),
\end{array}\right.
$$

where $a_{i}(t), b_{i}(t)$, and $c_{i}(t)(i=1,2)$ are continuous and bounded above and below by positive constants.

Given a function $f(t)$, which is bounded above and below by positive constants, we let

$$
f^{L}=\inf _{t \in \mathbb{R}} f(t) \quad \text { and } \quad f^{M}=\sup _{t \in \mathbb{R}} f(t) .
$$

Lemma 2.6. Suppose that $a_{1}^{L}>\frac{c_{1}^{M} a_{2}^{M}}{c_{2}^{L}}$ and $a_{2}^{L}>\frac{a_{1}^{M} b_{2}^{M}}{b_{1}^{L}}$.

(1) Suppose that $\left(u_{1}(t), v_{1}(t)\right)$ and $\left(u_{2}(t), v_{2}(t)\right)$ are two solutions of the system (2.5) with $u_{k}\left(t_{0}\right)>0, v_{k}\left(t_{0}\right)>0(k=1,2)$. Then $u_{1}(t)-u_{2}(t) \rightarrow 0$ and $v_{1}(t)-v_{2}(t) \rightarrow 0$ as $t \rightarrow \infty$.

(2) For any $t_{0} \in \mathbb{R}$, there exists a solution $\left(u_{0}(t), v_{0}(t)\right)$ of system (2.5) for $t \geq t_{0}$ such that

$$
\begin{aligned}
& 0<\frac{a_{1}^{L} c_{2}^{L}-c_{1}^{M} a_{2}^{M}}{b_{1}^{M} c_{2}^{L}-c_{1}^{M} b_{2}^{L}} \leq u_{0}(t) \leq \frac{a_{1}^{M} c_{2}^{M}-c_{1}^{L} a_{2}^{L}}{b_{1}^{L} c_{2}^{M}-c_{1}^{L} b_{2}^{M}} \quad \forall t \geq t_{0}, \\
& 0<\frac{b_{1}^{L} a_{2}^{L}-a_{1}^{M} b_{2}^{M}}{b_{1}^{L} c_{2}^{M}-c_{1}^{L} b_{2}^{M}} \leq v_{0}(t) \leq \frac{b_{1}^{M} a_{2}^{M}-a_{1}^{L} b_{2}^{L}}{b_{1}^{M} c_{2}^{L}-c_{1}^{M} b_{2}^{L}} \quad \forall t \geq t_{0} .
\end{aligned}
$$


(3) If moreover the coefficients are positive and T-periodic, then there exist exactly one Tperiodic solution of the system (2.5) with positive components, which attracts all solutions that begin in the open first quadrant.

(4) If moreover the coefficients are positive and almost periodic, then there exist exactly one almost periodic solution of the system (2.5) with positive components, which attracts all solutions that begin in the open first quadrant.

Proof. (1), (2), (3) follow from [1, Theorems 1 and 2], and (4) follows from [16, Theorem C].

\section{Local existence and uniqueness of classical solutions}

In this section, we study the local existence and uniqueness of classical solutions of (1.3) with given initial functions and prove Theorem 1.1.

First, observe that $C^{0}(\bar{\Omega}) \subset L^{p}(\Omega)$ for any $1 \leq p<\infty$. Throughout this section, unless specified otherwise, $p>1$ and $\alpha \in(1 / 2,1)$ are such that $X^{\alpha} \subset C^{1}(\bar{\Omega})$, where $X^{\alpha}=D\left(A^{\alpha}\right)$ with the graph norm $\|u\|_{\alpha}=\left\|A^{\alpha} u\right\|_{L^{p}(\Omega)}$ and $A=I-\Delta$ with domain $D(A)=\left\{u \in W^{2, p}(\Omega) \mid \frac{\partial u}{\partial n}=0\right.$ on $\partial \Omega\}$. Note that $A: D(A) \rightarrow X^{0}\left(=L^{p}(\Omega)\right)$ is a linear, bounded bijection, and $A^{-1}: X^{0} \rightarrow X^{\alpha}$ is compact.

Next, we note that if $\left(u\left(x, t ; t_{0}, u_{0}\right), v\left(x, t ; t_{0}, u_{0}\right)\right)$ is a classical solution of (1.3) satisfying the properties in Theorem $1.1(1)$ or $(2)$, then $v\left(\cdot, t ; t_{0}, u_{0}\right)=A^{-1} u\left(\cdot, t ; t_{0}, u_{0}\right)$ and $u\left(x, t ; t_{0}, u_{0}\right)$ is a classical solution of

$$
\left\{\begin{array}{l}
u_{t}=(\Delta-1) u+f(t, x, u), \quad x \in \Omega \\
\frac{\partial u}{\partial n}=0, \quad x \in \partial \Omega
\end{array}\right.
$$

with $u\left(x, t_{0} ; t_{0}, u_{0}\right)=u_{0}(x)$, where

$$
f(t, x, u)=-\chi \nabla u \cdot \nabla A^{-1} u+\chi u\left(u-A^{-1} u\right)+u\left(1+a_{0}(t, x)-a_{1}(t, x) u-a_{2}(t, x) \int_{\Omega} u\right) .
$$

Conversely, if $u_{0} \in X^{\alpha}$ (resp. $\left.u_{0} \in C^{0}(\bar{\Omega})\right)$ and $u\left(x, t ; t_{0}, u_{0}\right)$ is a classical solution of (3.1) satisfying the properties in Theorem $1.1(1)$ (resp. (2)), then $\left(u\left(x, t ; t_{0}, u_{0}\right), v\left(x, t ; t_{0}, u_{0}\right)\right)$ is a classical solution of (1.3) satisfying the properties in Theorem 1.1 (1) (resp. (2)), where $v\left(\cdot, t ; t_{0}, u_{0}\right)=A^{-1} u\left(\cdot, t ; t_{0}, u_{0}\right)$.

We now prove Theorem 1.1 and we will only present the proof for $\chi>0$ since the case $\chi \leq 0$ is similar. In the rest of this section, $C$ denotes a constant independent of the initial conditions and the solutions under consideration, unless otherwise specified.

Proof of Theorem 1.1. (1) We use the semigroup approach to prove (1) and divide the proof into four steps.

Step 1. (Existence of strong solution). In this step, we prove the existence of a unique strong solution $u\left(\cdot, t ; t_{0}, u_{0}\right)$ of (3.1) in $X^{\alpha}$ with $u\left(\cdot, t_{0} ; t_{0}, u_{0}\right)=u_{0}$ and satisfying (1.6) and (1.7). In order to do so, we write (3.1) as

$$
u_{t}+A u=F(t, u)
$$


where $F(t, u)=-\chi \nabla u \cdot \nabla A^{-1} u+\chi u\left(u-A^{-1} u\right)+u\left(1+a_{0}(t, \cdot)-a_{1}(t, \cdot) u-a_{2}(t, \cdot) \int_{\Omega} u\right)$.

We claim that $F: \mathbb{R} \times X^{\alpha} \rightarrow X^{0}$ is locally Hölder continuous in $t$ and locally Lipschitz continuous in $u$. In fact, for any $s, t \in \mathbb{R}$ and $u, w \in X^{\alpha}$, we have

$$
\|F(t, u)-F(s, w)\|_{L^{p}(\Omega)} \leq C\left[\left(\|u\|_{C^{1}}+\|w\|_{C^{1}}+1\right)\|u-w\|_{C^{1}}+\left(\|u\|_{C^{1}}+\|w\|_{C^{1}}^{2}+\|w\|_{C^{1}}\right)|t-s|^{\nu}\right] .
$$

Since $X^{\alpha} \subset C^{1}(\bar{\Omega})$, we get

$$
\|F(t, u)-F(s, w)\|_{L^{p}(\Omega)} \leq C\left[\left(\|u\|_{\alpha}+\|w\|_{\alpha}+1\right)\|u-w\|_{\alpha}+\left(\|u\|_{\alpha}+\|w\|_{\alpha}^{2}+\|w\|_{\alpha}\right)|t-s|^{\nu}\right] .
$$

The claim then follows. Then by Lemma $2.3(1)$, for every $\left(t_{0}, u_{0}\right) \in \mathbb{R} \times X^{\alpha}$, there exists $T_{\max }=$ $T_{\max }\left(t_{0}, u_{0}\right)>0$ such that (3.2) has a unique strong solution $u\left(\cdot, t ; t_{0}, u_{0}\right)$ on $\left(t_{0}, t_{0}+T_{\max }\right)$. Moreover if $T_{\max }<\infty$, then $\lim \sup _{t} T_{\max }\left\|u\left(\cdot, t+t_{0} ; t_{0}, u_{0}\right)\right\|_{X^{\alpha}}=\infty$.

Note that $u\left(\cdot, \cdot ; t_{0}, u_{0}\right) \in C\left(\left[t_{0}, t_{0}+T_{\max }\right), X^{\alpha}\right)$. By Lemma 2.3 $(2), u \in C^{\delta}\left(\left(t_{0}, t_{0}+T_{\max }\right), X^{\alpha}\right) \cap$ $C^{1}\left(\left(t_{0}, t_{0}+T_{\max }\right), X_{0}\right)$ for any $\delta$ satisfying $0<\delta<1-\alpha$. Hence (1.6) holds. Moreover, $u(x, t):=u\left(x, t ; t_{0}, u_{0}\right)$ is a mild solution of (3.2) given by

$$
\begin{aligned}
u(\cdot, t)= & e^{-A\left(t-t_{0}\right)} u_{0}-\chi \int_{t_{0}}^{t} e^{-A(t-s)} \nabla u(\cdot, s) \cdot \nabla A^{-1} u(\cdot, s) d s \\
& +\chi \int_{t_{0}}^{t} e^{-A(t-s)} u(\cdot, s)\left(u(\cdot, s)-A^{-1} u(\cdot, s)\right) d s \\
& +\int_{t_{0}}^{t} e^{-A(t-s)} u(\cdot, s)\left(1+a_{0}(s, \cdot)-a_{1}(s, \cdot) u(\cdot, s)-a_{2}(s, \cdot) \int_{\Omega} u(\cdot, s)\right) d s .
\end{aligned}
$$

It remains to show that, if $T_{\max }<\infty$, then (1.7) holds. Assume by contradiction that $T_{\max }<\infty$ and $\lim \sup _{t} T_{\max }\left(\left\|u\left(\cdot, t+t_{0}\right)\right\|_{C^{0}(\bar{\Omega})}\right)=L<\infty$. Then there exists $\delta_{0}>0$ such that

$$
L \leq \sup _{T_{\max }-\delta_{0}<t<T_{\max }}\left\|u\left(\cdot, t+t_{0}\right)\right\|_{C^{0}(\bar{\Omega})}<L+1 .
$$

Let $t$ be such that $t_{0}+T_{\max }-\delta_{0}<t<t_{0}+T_{\max }$. We have

$$
\begin{aligned}
& \|F(t, u(\cdot, t))\|_{L^{p}(\Omega)} \\
& \leq\left\|-\chi \nabla u \cdot \nabla A^{-1} u\right\|_{L^{p}(\Omega)}+\left\|\chi u\left(u-A^{-1} u\right)+u\left(1+a_{0}(t, \cdot)-a_{1}(t, \cdot) u-a_{2}(t, \cdot) \int_{\Omega} u\right)\right\|_{L^{p}(\Omega)} \\
& \leq \chi\|u(\cdot, t)\|_{C^{1}(\bar{\Omega})}\left\|\nabla A^{-1} u(\cdot, t)\right\|_{L^{p}(\Omega)} \\
& +\|u(\cdot, t)\|_{C^{0}(\bar{\Omega})}\left(A_{0}+1+|\Omega|\left(\chi+A_{1}+|\Omega| A_{2}\right)\|u(\cdot, t)\|_{C^{0}(\bar{\Omega})}+\chi\left\|A^{-1} u(\cdot, t)\right\|_{L^{p}(\Omega)}\right) .
\end{aligned}
$$

By the regularity and a priori estimates for parabolic equations, (3.3), and $X^{\alpha} \subset C^{1}(\bar{\Omega})$, there a positive constant $C=C(L)$ independent of $t$ such that

$$
\|F(t, u(\cdot, t))\|_{L^{p}(\Omega)} \leq C\left(1+\|u(\cdot, t)\|_{\alpha}\right) .
$$

Fix $t_{1}$ such that $t_{0}+T_{\max }-\delta_{0}<t_{1}<t_{0}+T_{\max }$. Note that, on $\left[t_{1}, t_{0}+T_{\max }\right)$, we have

$$
u(\cdot, t)=e^{-A\left(t-t_{1}\right)} u\left(\cdot, t_{1}\right)+\int_{t_{1}}^{t} e^{-A(t-s)} F(s, u(\cdot, s)) d s .
$$


By [11, Theorem 1.4.3], we get

$$
\begin{aligned}
\|u(\cdot, t)\|_{\alpha} & \leq\left\|e^{-A\left(t-t_{1}\right)} u\left(\cdot, t_{1}\right)\right\|_{\alpha}+\chi \int_{t_{1}}^{t}\left\|A^{\alpha} e^{-A(t-s)}\right\|\|F(s, u(\cdot, s))\|_{p} d s \\
& \leq C\left(t-t_{1}\right)^{-\alpha}\left\|u\left(\cdot, t_{1}\right)\right\|_{C^{0}(\bar{\Omega})}+C \int_{t_{1}}^{t}(t-s)^{-\alpha}\left(1+\|u(\cdot, s)\|_{\alpha}\right) d s \\
& \leq\left(C\left\|u\left(\cdot, t_{1}\right)\right\|_{C^{0}(\bar{\Omega})}+C\left(T_{\max }-t_{0}\right)\right)\left(t-t_{1}\right)^{-\alpha}+C \int_{t_{1}}^{t}(t-s)^{-\alpha}\|u(\cdot, s)\|_{\alpha} d s .
\end{aligned}
$$

Then by Gronwall's inequality (see [11, page 6]), there exists a constant $M=M\left(C\left\|u\left(\cdot, t_{1}\right)\right\|_{C^{0}(\bar{\Omega})}+\right.$ $\left.C\left(T_{\max }-t_{1}\right), \alpha, T_{\max }\right)$ such that

$$
\|u(\cdot, t)\|_{\alpha} \leq\left\{C\left\|u\left(\cdot, t_{1}\right)\right\|_{C^{0}(\bar{\Omega})}+C\left(T_{\max }-t_{1}\right)\right\} M\left(t-t_{1}\right)^{-\alpha} .
$$

Thus $\lim \sup _{t} \nearrow T_{\max }\left\|u\left(\cdot, t+t_{0}\right)\right\|_{X^{\alpha}}<\infty$, a contradiction. Hence if $T_{\max }<\infty$, then (1.7) holds.

Step 2. (Regularity). In this step, we prove that $u(x, t):=u\left(x, t ; t_{0}, u_{0}\right)$ obtained in (i) is a classical solution of (3.1) on $\left(t_{0}, t_{0}+T_{\max }\right)$ and then $\left(u\left(x, t ; t_{0}, u_{0}\right), v\left(x, t ; t_{0}, u_{0}\right)\right)$ is a classical solution of (1.3) on $\left(t_{0}, t_{0}+T_{\max }\right)$ satisfying the properties in Theorem 1.1(1), where $v\left(\cdot, t ; t_{0}, u_{0}\right)=A^{-1} u\left(\cdot, t ; t_{0}, u_{0}\right)$.

Fix $t_{0}<t_{1}<T<t_{0}+T_{\max }$ and consider the problem

$$
\begin{cases}\tilde{u}_{t}(x, t)=(\Delta-1) \tilde{u}(x, t)+g(x, t), & x \in \Omega, \quad t \in\left(t_{1}, T\right) \\ \tilde{u}\left(x, t_{1}\right)=u\left(x, t_{1}\right), & x \in \Omega \\ \frac{d \tilde{u}}{d n}=0, \quad x \in \partial \Omega, & \end{cases}
$$

where

$$
\begin{aligned}
g(x, t)= & -\chi \nabla A^{-1} u(x, t) \cdot \nabla u(x, t) \\
& +\left(1+a_{0}(x, t)-\chi A^{-1} u(x, t)+\chi u(x, t)-a_{1}(x, t) u(x, t)-a_{2}(x, t) \int_{\Omega} u(\cdot, t)\right) u(x, t) .
\end{aligned}
$$

By Lemma 2.1, $t \rightarrow g(\cdot, t) \in C^{\theta}(\bar{\Omega})$ is Hölder continuous in $t \in\left(t_{0}, t_{0}+T_{\max }\right)$ for some $\theta \in(0,1)$. Then by [2, Theorem 15.1, Corollary 15.3], (3.4) has a unique classical solution $\tilde{u} \in C^{2,1}(\bar{\Omega} \times$ $\left.\left(t_{1}, T\right)\right) \cap C^{0}\left(\bar{\Omega} \times\left[t_{1}, T\right)\right)$. Moreover, by Lemma 2.3 ,

$$
\begin{aligned}
\tilde{u}(\cdot, t)= & e^{-A\left(t-t_{1}\right)} u\left(\cdot, t_{1}\right)-\chi \int_{t_{1}}^{t} e^{-A(t-s)}\left(\nabla u(\cdot, s) \cdot \nabla A^{-1} u(\cdot, s)-u(\cdot, s)\left(u(\cdot, s)-A^{-1} u(\cdot, s)\right)\right) d s \\
& +\int_{t_{1}}^{t} e^{-A(t-s)} u(\cdot, s)\left(1+a_{0}(s, \cdot)-a_{1}(s, \cdot) u(\cdot, s)-a_{2}(s, \cdot) \int_{\Omega} u(\cdot, s)\right) d s .
\end{aligned}
$$

Thus $\tilde{u}(x, t)=u(x, t)$ for $t \in\left[t_{1}, T\right)$ and $u \in C^{2,1}\left(\bar{\Omega} \times\left(t_{1}, T\right)\right) \cap C^{0}\left(\bar{\Omega} \times\left[t_{1}, T\right)\right)$. Letting $t_{1} \rightarrow t_{0}$ and $T \rightarrow T_{\max }$, we have $u \in C^{2,1}\left(\bar{\Omega} \times\left(t_{0}, t_{0}+T_{\max }\right)\right) \cap C^{0}\left(\bar{\Omega} \times\left[t_{0}, t_{0}+T_{\max }\right)\right)$.

Let $v\left(\cdot, t ; t_{0}, u_{0}\right)=A^{-1} u\left(\cdot, t ; t_{0}, u_{0}\right)$. We then have that $\left(u\left(x, t ; t_{0}, u_{0}\right), v\left(x, t ; t_{0}, u_{0}\right)\right)$ is a classical solution of (1.3) on $\left(t_{0}, t_{0}+T_{\max }\right)$ satisfying the properties in Theorem 1.1 .

Step 3. (Uniqueness). In this step, we prove the uniqueness of classical solutions of (1.3) satisfying the properties in Theorem 1.1(1). 
Suppose that $\left(u_{1}(x, t), v_{1}(x, t)\right)$ and $\left(u_{2}(x, t), v_{2}(x, t)\right)$ are two classical solutions of (1.3) on $\left(t_{0}, t_{0}+T_{\max }\right)$ satisfying the properties in Theorem 1.1. First, set $u=u_{1}-u_{2}$ and $v=v_{1}-v_{2}$. Then $(u, v)$ satisfies

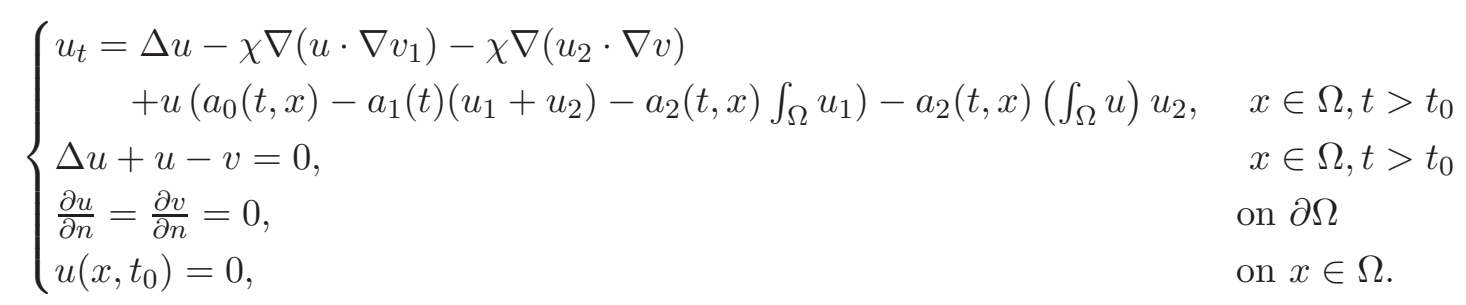

Next, fix $t_{1}, T$ such that $t_{0}<t_{1}<T<t_{0}+T_{\max }$. It is clear that, for $t \in\left[t_{1}, t_{0}+T_{\max }\right)$,

$$
\begin{aligned}
u(\cdot, t)= & e^{-A\left(t-t_{1}\right)}\left(u_{1}\left(\cdot, t_{1}\right)-u_{2}\left(\cdot, t_{1}\right)\right)-\chi \int_{t_{1}}^{t} e^{-A(t-s)} \nabla\left[u(\cdot, s) \cdot \nabla v_{1}(\cdot, s)+u_{2}(\cdot, s) \cdot \nabla v(\cdot, s)\right] d s \\
& +\int_{t_{1}}^{t} e^{-A(t-s)} u(\cdot, s)\left(1+a_{0}(s, \cdot)-a_{1}(s, \cdot)\left(u_{1}(\cdot, s)+u_{1}(\cdot, s)\right)-a_{2}(s, \cdot) \int_{\Omega} u_{1}(\cdot, s)\right) d s \\
& -\int_{t_{1}}^{t} e^{-A(t-s)} a_{2}(s, \cdot)\left(\int_{\Omega} u(\cdot, s)\right) u_{2}(\cdot, s) d s .
\end{aligned}
$$

Now, fix $0<\beta<\frac{1}{2}$. By regularity and a priori estimates for elliptic equations, [11, Theorem 1.4.3], Lemma 2.2, and (3.5), for any $\epsilon \in\left(0, \frac{1}{2}-\beta\right)$, we have

$$
\begin{aligned}
\|u(\cdot, t)\|_{X^{\beta}} \leq & C\left\|u\left(\cdot, t_{1}\right)\right\|_{X^{\beta}}+C \chi \max _{t_{1} \leq s \leq T}\left\|\nabla v_{1}(\cdot, s)\right\|_{C^{0}(\bar{\Omega})} \int_{t_{1}}^{t}(t-s)^{-\beta-\epsilon-\frac{1}{2}}\|u(\cdot, s)\|_{X^{\beta}} d s \\
& +C \chi \max _{t_{1} \leq s \leq T}\left\|u_{2}(\cdot, s)\right\|_{C^{0}(\bar{\Omega})} \int_{t_{1}}^{t}(t-s)^{-\beta-\epsilon-\frac{1}{2}}\|u(\cdot, s)\|_{X^{\beta}} d s \\
& +C \int_{t_{1}}^{t}(t-s)^{-\beta}\left\{1+A_{0}+A_{1}\left[\max _{t_{1} \leq s \leq T}\left(\left\|u_{1}(\cdot, s)\right\|_{C^{0}(\bar{\Omega})}+\left\|u_{2}(\cdot, s)\right\|_{C^{0}(\bar{\Omega})}\right)\right]\right\}\|u(\cdot, s)\|_{X^{\beta}} d s \\
& +C \int_{t_{1}}^{t}(t-s)^{-\beta} A_{2}|\Omega| \max _{t_{1} \leq s \leq T}\left\|u_{1}(\cdot, s)\right\|_{C^{0}(\bar{\Omega})}\|u(\cdot, s)\|_{X^{\beta}} d s \\
& +C \int_{t_{1}}^{t}(t-s)^{-\beta} A_{2}\left\|u_{2}(\cdot, s)\right\|_{C^{0}(\bar{\Omega})}\|u(\cdot, s)\|_{X^{\beta}} d s .
\end{aligned}
$$

Thus there exists a constant positive $C=C\left(u_{1}, u_{2}, v_{1}, v_{2}, A_{i}, T, \beta, \epsilon\right)$ such that for all $t \in\left[t_{1}, T\right]$,

$$
\|u(\cdot, t)\|_{X^{\beta}} \leq C_{1}\left\|u\left(\cdot, t_{1}\right)\right\|_{X^{\beta}}+C \int_{t_{1}}^{t}(t-s)^{-\beta-\epsilon-\frac{1}{2}}\|u(\cdot, s)\|_{X^{\beta}} d s .
$$

Letting $t_{1} \rightarrow t_{0}$, we have

$$
\|u(\cdot, t)\|_{X^{\beta}} \leq C \int_{t_{1}}^{t}(t-s)^{-\beta-\epsilon-\frac{1}{2}}\|u(\cdot, s)\|_{X^{\beta}} d s \quad \text { for } \quad t \in\left[t_{0}, T\right] .
$$

By (3.7) and the generalized Gronwall's inequality (see [11, page 6]), we get $u(\cdot, t)=0$ for $t \in\left[t_{0}, T\right]$. Letting $T \rightarrow t_{0}+T_{\max }$, we get $u(\cdot, t)=0$ for $t \in\left[t_{0}, t_{0}+T_{\max }\right)$. Since $v(\cdot, t)=$ $A^{-1} u(\cdot, t), v(\cdot, t)=0$ for $t \in\left[t_{0}, t_{0}+T_{\max }\right)$. Therefore $\left(u_{1}(x, t), v_{1}(x, t)\right)=\left(u_{2}(x, t), v_{2}(x, t)\right)$ for $(x, t) \in \bar{\Omega} \times\left[t_{0}, t_{0}+T_{\max }\right)$. 
Step 4. (Nonnegativity). In this last step, we prove the nonnegativity of the classical solutions. Since $u\left(x, t ; t_{0}, u_{0}\right)$ is classical solution of (3.1) , by maximum principle for parabolic equations, we have that $u\left(x, t ; t_{0}, u_{0}\right)$ is nonnegative (see [8, Theorem 7 on page 41]). And now, since $u\left(x, t ; t_{0}, u_{0}\right)$ is nonnegative, by maximum principle for elliptic equations, $v\left(x, t ; t_{0}, u_{0}\right)$ is nonnegative (see [8, Theorem 18 on page 53]).

(2) We prove (2) by Banach Fixed Point Theorem and some arguments in (1) and divide the proof into three steps. To this end, we first introduce the notion of generalized mild solution of (3.2). A function $u \in C^{0}\left(\left[t_{0}, t_{0}+T\right), C^{0}(\bar{\Omega})\right)$ is called a generalized mild solution of (3.2) with $u\left(t_{0}\right)=u_{0}$ if

$$
\begin{aligned}
u(t)= & e^{-A\left(t-t_{0}\right)} u_{0}-\chi \int_{t_{0}}^{t} e^{-A(t-s)} \nabla \cdot\left(u(s) \nabla A^{-1} u(s)\right) d s \\
& +\int_{t_{0}}^{t} e^{-A(t-s)} u(s)\left(1+a_{0}(s, \cdot)-a_{1}(s, \cdot) u(s)-a_{2}(s, \cdot) \int_{\Omega} u(s)\right) d s
\end{aligned}
$$

for $t \in\left[t_{0}, t_{0}+T\right)$.

Step 1. (Existence of generalized mild solution). In this step, we prove the existence of a unique generalized mild solution $u\left(\cdot, t ; t_{0}, u_{0}\right)$ of $(\underline{3.2})$.

In order to do so, fix $t_{0} \in \mathbb{R}$ and $u_{0} \in C^{0}(\bar{\Omega})$. For given $T>0$ and $R>\left\|u_{0}\right\|_{C^{0}(\bar{\Omega})}$, let

$$
\mathcal{X}_{T}=C^{0}\left(\left[t_{0}, t_{0}+T\right], C^{0}(\bar{\Omega})\right)
$$

with the supremum norm $\|u\|_{\mathcal{X}_{T}}=\max _{t_{0} \leq t \leq t_{0}+T}\|u(t)\|_{C^{0}(\bar{\Omega})}$, and let

$$
\mathcal{S}_{T, R}=\left\{u \in \mathcal{X}_{T} \mid\|u\|_{\mathcal{X}_{T}} \leq R\right\} .
$$

Note that $\mathcal{S}_{T, R}$ is a closed subset of the Banach space $\mathcal{X}_{T}$.

First, we claim that, for given $u \in \mathcal{S}_{T, R}$ and $t \in\left[t_{0}, t_{0}+T\right],(G u)(t)$ is well defined, where

$$
\begin{aligned}
(G u)(t)= & e^{-A\left(t-t_{0}\right)} u_{0}-\chi \int_{t_{0}}^{t} e^{-A(t-s)} \nabla \cdot\left(u(s) \nabla A^{-1} u(s)\right) d s \\
& +\int_{t_{0}}^{t} e^{-A(t-s)} u(s)\left(1+a_{0}(s, \cdot)-a_{1}(s, \cdot) u(s)-a_{2}(s, \cdot) \int_{\Omega} u(s)\right) d s
\end{aligned}
$$

and the integrals are taken in $C^{0}(\bar{\Omega})$. In fact, for any $u \in \mathcal{S}_{T, R}$, there is $\left\{u_{n}\right\} \subset \mathcal{S}_{T, R}$ satisfying that $\frac{\partial u_{n}}{\partial x_{i}} \in \mathcal{S}_{T, R}, \frac{\partial^{2} u_{n}}{\partial x_{i} \partial x_{j}} \in \mathcal{S}_{T, R}$ and $\left\|u_{n}-u\right\|_{\mathcal{X}_{T}} \rightarrow 0 \quad$ as $n \rightarrow \infty$. This implies that

$$
\begin{aligned}
\left(t_{0}, t\right) \ni s \rightarrow & e^{-A(t-s)} \nabla \cdot\left(u(s) \nabla A^{-1} u(s)\right) \\
& \quad+e^{-A(t-s)} u(s)\left(1+a_{0}(s, \cdot)-a_{1}(s, \cdot) u(s)-a_{2}(s, \cdot) \int_{\Omega} u(s)\right) \in C^{0}(\bar{\Omega})
\end{aligned}
$$

is measurable. Moreover, choose $p$ such that $n<p$ and then choose $\beta$ such that $\frac{n}{2 p}<\beta<\frac{1}{2}$. 
By Lemma 2.1, we get $X^{\beta} \subset C^{0}(\bar{\Omega})$. Then we have

$$
\begin{aligned}
\|G(u)(t)\|_{C^{0}(\bar{\Omega})} & \leq\left\|e^{-A\left(t-t_{0}\right)} u_{0}\right\|_{C^{0}(\bar{\Omega})}+\chi \int_{t_{0}}^{t}\left\|e^{-A(t-s)} \nabla \cdot\left(u(s) \nabla A^{-1} u(s)\right)\right\|_{C^{0}(\bar{\Omega})} d s \\
& +\int_{t_{0}}^{t}\left\|e^{-A(t-s)} u(s)\left(1+a_{0}(s, \cdot)-a_{1}(s, \cdot) u(s)-a_{2}(s, \cdot) \int_{\Omega} u(s)\right)\right\|_{C^{0}(\bar{\Omega}} d s \\
& \leq\left\|u_{0}\right\|_{C^{0}(\bar{\Omega})}+C \int_{t_{0}}^{t}\left\|A^{\beta} e^{-A(t-s)} \nabla \cdot\left(u(s) \nabla A^{-1} u(s)\right)\right\|_{L^{p}(\Omega)} d s \\
& +C \int_{t_{0}}^{t}\left\|A^{\beta} e^{-A(t-s)} u(s)\left(1+a_{0}(s, \cdot)-a_{1}(s, \cdot) u(s)-a_{2}(s) \int_{\Omega} u(s)\right)\right\|_{L^{p}(\Omega)} d s
\end{aligned}
$$

for $t \in\left[t_{0}, t_{0}+T\right]$. By regularity and a priori estimates for parabolic equations and Lemma 2.2 , for any $\epsilon \in\left(0, \frac{1}{2}-\beta\right)$, we have

$$
\begin{aligned}
\|G(u)(t)\|_{C^{0}(\bar{\Omega}) \leq} & \left\|u_{0}\right\|_{C^{0}(\bar{\Omega})}+C R^{2} \int_{t_{0}}^{t}(t-s)^{-\beta-\frac{1}{2}-\epsilon} e^{-\mu(t-s)} d s \\
& +C R\left[1+A_{0}+R\left(A_{1}+|\Omega| A_{2}\right)\right] \int_{t_{0}}^{t}(t-s)^{-\beta} e^{-\mu(t-s)} d s \\
\leq & \left\|u_{0}\right\|_{C^{0}(\bar{\Omega})}+C R^{2} T^{\frac{1}{2}-\beta-\epsilon}+C R\left[1+A_{0}+R\left(A_{1}+|\Omega| A_{2}\right)\right] T^{1-\beta}
\end{aligned}
$$

for $t \in\left[t_{0}, t_{0}+T\right]$. The claim then follows.

Next, fix $R>\left\|u_{0}\right\|_{C^{0}(\bar{\Omega})}$. We claim that $G$ maps $\mathcal{S}_{T, R}$ into itself and is a contraction for $0<T \ll 1$.

We first show that $G$ maps $\mathcal{S}_{T, R}$ into itself for $0<T \ll 1$. To this end, for any $u \in \mathcal{S}_{T, R}$, $0 \leq \beta<\frac{1}{2}$, and $t_{0}<t<t_{0}+T$, we have

$$
\begin{aligned}
\|(G u)(t)\|_{X^{\beta}} & \leq\left\|A^{\beta} e^{-A\left(t-t_{0}\right)} u_{0}\right\|_{L^{p}(\Omega)}+\chi \int_{t_{0}}^{t}\left\|A^{\beta} e^{-A(t-s)} \nabla \cdot\left(u(s) \nabla A^{-1} u(s)\right)\right\|_{L^{p}(\Omega)} d s \\
& +\int_{t_{0}}^{t}\left\|A^{\beta} e^{-A(t-s)} u(s)\left(1+a_{0}(s, \cdot)-a_{1}(s, \cdot) u(s)-a_{2}(s, \cdot) \int_{\Omega} u(s)\right)\right\|_{L^{p}(\Omega)} d s .
\end{aligned}
$$

Then by [11, Theorem 1.4.3] and Lemma 2.2, for any $\epsilon \in\left(0, \frac{1}{2}-\beta\right)$, we have

$$
\|(G u)(t))\left\|_{X^{\beta}} \leq C\right\| u_{0} \|_{C^{0}(\bar{\Omega})}\left(t-t_{0}\right)^{-\beta}+C R^{2}\left(t-t_{0}\right)^{\frac{1}{2}-\beta-\epsilon}+C R\left[1+A_{0}+R\left(A_{1}+|\Omega| A_{2}\right)\right]\left(t-t_{0}\right)^{1-\beta} .
$$

Thus $u(t) \in X^{\beta}$. Choose $\beta$ such $\frac{n}{2 p}<\beta<\frac{1}{2}$ and then choose $\theta$ such that $0<\theta<2 \beta-\frac{n}{p}$, by Lemma 2.2, we have that $X^{\beta} \subset C^{\theta}(\bar{\Omega})$. Thus $u(t) \in C^{\theta}(\bar{\Omega})$ for any $t_{0}<t<t_{0}+T$.

Choose $\sigma$ such that $\beta+\sigma<\frac{1}{2}$ where $\beta$ is as in the above. Fix any $t_{0}<T<t_{0}+T_{\max }$. Then 
for any $t, h>0$ such that $t_{0}<t<t+h<T<t_{0}+T$,

$$
\begin{aligned}
(G u)(t+h)-(G u)(t) & =\underbrace{\left(e^{-A h}-I\right) e^{-A\left(t-t_{0}\right)} u_{0}}_{I_{1}}-\chi \underbrace{\int_{t_{0}}^{t}\left(e^{-A h}-I\right) e^{-A(t-s)} \nabla \cdot\left(u(s) \nabla A^{-1} u(s)\right) d s}_{I_{3}} \\
& +\underbrace{\int_{t_{0}}^{t}\left(e^{-A h}-I\right) e^{-A(t-s)} u(s)\left(1+a_{0}(s, \cdot)-a_{1}(s, \cdot) u(s)-a_{2}(s, \cdot) \int_{\Omega} u(s)\right) d s}_{I_{4}} \\
& -\chi \underbrace{\int_{t}^{t+h} e^{-A(t+h-s)} \nabla \cdot\left(u(s) \nabla A^{-1} u(s)\right) d s}_{I_{5}} \\
& +\chi \underbrace{\int_{t}^{t+h} e^{-A(t+h-s)} u(s)\left(1+a_{0}(s, \cdot)-a_{1}(s, \cdot) u(s)-a_{2}(s, \cdot) \int_{\Omega} u(s)\right) d s} .
\end{aligned}
$$

Since $e^{-A\left(t-t_{0}\right)} u_{0} \in D(A)$, we have

$$
A^{\beta}\left(e^{-A h}-I\right) A^{-\sigma}\left(A^{\sigma} e^{-A\left(t-t_{0}\right)} u_{0}\right)=\left(e^{-A h}-I\right) A^{-\sigma}\left(A^{\sigma+\beta} e^{-A\left(t-t_{0}\right)} u_{0}\right) .
$$

Therefore by [11, Theorem 1.4.3], we get

$$
\begin{aligned}
\left\|I_{1}\right\|_{X^{\beta}} & =\left\|\left(e^{-A h}-I\right) A^{-\sigma}\left(A^{\sigma+\beta} e^{-A\left(t-t_{0}\right)} u_{0}\right)\right\|_{L^{p}(\Omega)} \leq C h^{\sigma}\left\|A^{\sigma+\beta} e^{-A\left(t-t_{0}\right)} u_{0}\right\|_{L^{p}(\Omega)} \\
& \leq C h^{\sigma}\left(t-t_{0}\right)^{-\sigma-\beta} e^{-\left(t-t_{0}\right)}\left\|u_{0}\right\|_{C^{0}(\bar{\Omega})} .
\end{aligned}
$$

By [11, Theorem 1.4.3] and Lemma 2.2, for any $\epsilon \in\left(0, \frac{1}{2}-\beta\right)$, we have

$$
\begin{aligned}
\left\|I_{2}\right\|_{X^{\beta}} & \leq \int_{t_{0}}^{t}\left\|\left(e^{-A h}-I\right) A^{-\sigma}\left(A^{\sigma+\beta} e^{-A(t-s)} \nabla \cdot\left(u(s) \nabla A^{-1} u(s)\right)\right)\right\|_{L^{p}(\Omega)} d s \\
& \leq C h^{\sigma} \int_{t_{0}}^{t}\left\|A^{\sigma+\beta} e^{-A(t-s)} \nabla \cdot\left(u(s) \nabla A^{-1} u(s)\right)\right\|_{L^{p}(\Omega)} d s \\
& \leq C h^{\sigma} \int_{t_{0}}^{t}(t-s)^{-\beta-\sigma-\frac{1}{2}-\epsilon}\left\|u(s) \nabla A^{-1} u(s)\right\|_{L^{p}(\Omega)} d s \\
& \leq C h^{\sigma} \sup _{t_{0} \leq s \leq T}\|u(s)\|_{C^{0}(\bar{\Omega})}^{2}\left(t-t_{0}\right)^{\frac{1}{2}-\beta-\sigma-\epsilon} .
\end{aligned}
$$

By similar arguments as in the estimates of $I_{2}$, we have

$$
\left\|I_{3}\right\|_{X^{\beta}} \leq C h^{\sigma} \sup _{t_{0} \leq s \leq T}\|u(s)\|_{C^{0}(\bar{\Omega})}\left(1+\sup _{t_{0} \leq s \leq T}\|u(s)\|_{C^{0}(\bar{\Omega})}\right)\left(t-t_{0}\right)^{\frac{1}{2}-\beta-\sigma-\epsilon} .
$$

By Lemma 2.2, we have

$$
\begin{aligned}
\left\|I_{4}\right\|_{X^{\beta}} & \leq \int_{t}^{t+h}\left\|A^{\beta} e^{-A(t+h-s)} \nabla \cdot\left(u(s) \nabla A^{-1} u(s)\right)\right\|_{L^{p}(\Omega)} d s \\
& \leq C \int_{t}^{t+h}(t+h-s)^{-\beta}\left\|u(s) \nabla A^{-1} u(s)\right\|_{L^{p}(\Omega)} d s \leq C h^{1-\beta} \sup _{t_{0} \leq s \leq T}\|u(s)\|_{C^{0}(\bar{\Omega})}^{2} .
\end{aligned}
$$


Similarly, we have

$$
\left\|I_{5}\right\|_{X^{\beta}} \leq C h^{1-\beta} \sup _{t_{0} \leq s \leq T}\|u(s)\|_{C^{0}(\bar{\Omega})}\left(1+\sup _{t_{0} \leq s \leq T}\|u(s)\|_{C^{0}(\bar{\Omega})}\right) .
$$

Therefore, $(G u)(t)$ is locally Hölder continuous from $\left(t_{0}, t_{0}+T\right)$ to $X^{\beta}$ with exponent $\sigma$. It is clear that $(G u)(t)$ is continuous at $t_{0}$ in $C^{0}(\bar{\Omega})$. Therefore $(G u)(t)$ is continuous in $t \in\left[t_{0}, t_{0}+T\right]$ in $C^{0}(\bar{\Omega})$. Then by (3.8),$G$ maps $\mathcal{S}_{T, R}$ into itself for $0<T \ll 1$.

We next show that $G$ maps $\mathcal{S}_{T, R}$ is a contraction for $0<T \ll 1$. To this end, for given $u, w \in \mathcal{S}_{T, R}$, we have

$$
\begin{aligned}
& \|G(u)(t)-G(w)(t)\|_{C^{0}(\bar{\Omega})} \\
& \leq C \int_{t_{0}}^{t}\left\|A^{\beta} e^{-A(t-s)} \nabla \cdot\left((u(s)-w(s)) \nabla A^{-1} u(s)+w(s) \nabla A^{-1}(u(s)-w(s))\right)\right\|_{L^{p}(\Omega)} d s \\
& +C \int_{t_{0}}^{t}\left\|A^{\beta} e^{-A(t-s)}(u(s)-w(s))\left(1+a_{0}(s, \cdot)-a_{1}(s, \cdot)(u(s)+w(s))-a_{2}(s, \cdot) \int_{\Omega} u(s)\right)\right\|_{L^{p}(\Omega)} d s \\
& +C \int_{t_{0}}^{t}\left\|A^{\beta} e^{-A(t-s)} a_{2}(s, \cdot) w(s) \int_{\Omega}(u(s)-w(s))\right\|_{L^{p}(\Omega)} d s \\
& \leq C R T^{\frac{1}{2}-\beta-\epsilon}\|u-w\|_{\mathcal{X}_{T}}+C R T^{1-\beta}\left(A_{0}+1+2 R\left(A_{1}+|\Omega| A_{2}\right)\right)\|u-w\|_{\mathcal{X}_{T}} .
\end{aligned}
$$

Then $G$ is a contraction for $0<T \ll 1$ and the claim follows.

Now, By Banach fixed point Theorem, $G$ has a unique fixed point $u \in \mathcal{S}_{T, R}$. That means $u \in C^{0}\left(\left[t_{0}, t_{0}+T\right], C^{0}(\bar{\Omega})\right)$ and

$$
\begin{aligned}
u(t)= & e^{-A\left(t-t_{0}\right)} u_{0}-\chi \int_{t_{0}}^{t} e^{-A(t-s)} \nabla \cdot\left(u(s) \nabla A^{-1} u(s)\right) d s \\
& +\int_{t_{0}}^{t} e^{-A(t-s)} u(s)\left(1+a_{0}(s, \cdot)-a_{1}(s, \cdot) u(s)-a_{2}(s, \cdot) \int_{\Omega} u(s)\right) d s .
\end{aligned}
$$

Hence $u\left(\cdot, t ; t_{0}, u_{0}\right):=u(t)(x)$ is a generalized mild solution of (3.2). The generalized mild solution $u\left(\cdot, t ; t_{0}, u_{0}\right)$ may be prolonged by standard method into a maximal interval $\left[t_{0}, t_{0}+T_{\max }\right)$ such that if $T_{\max }<\infty$ then $\lim \sup _{t} T_{\max }\left\|u\left(\cdot, t+t_{0} ; t_{0}, u_{0}\right)\right\|_{C^{0}(\bar{\Omega})}=\infty$.

Step 2. (Regularity). In this step, we prove that $u(t)=u\left(\cdot, t ; t_{0}, u_{0}\right)$ is a classical solution of (3.1) satisfying the properties in Theorem 1.1(2), where $\left.u\left(\cdot, t ; t_{0}, u_{0}\right)\right)$ is obtained in Step 1. Then $\left(u\left(x, t ; t_{0}, u_{0}\right), v\left(x, t ; t_{0}, u_{0}\right)\right)$ with $v\left(\cdot, t ; t_{0}, u_{0}\right)=A^{-1} u\left(\cdot, t ; t_{0}, u_{0}\right)$ is a classical solution of (1.3) satisfying the properties in Theorem 1.1 (2).

First, for any $0 \leq \beta<\frac{1}{2}$ and $\sigma$ such that $\beta+\sigma<\frac{1}{2}$, by the arguments in Step $1, u(t)$ is locally Hölder continuous from $\left(t_{0}, t_{0}+T_{\max }\right)$ to $X^{\beta}$ with exponent $\sigma$.

Next, fix $\frac{1}{2}<\alpha<1$. We define the map $B(t):\left(t_{0}, t_{0}+T_{\max }\right) \rightarrow \mathcal{L}\left(X^{\alpha}, L^{p}(\Omega)\right)$ by

$$
B(t) \tilde{u}=-\chi \nabla A^{-1} u(t) \cdot \nabla \tilde{u}+\left(a_{0}(t, \cdot)-\chi A^{-1} u(t)+\chi u(t)-a_{1}(t, \cdot) u(t)-a_{2}(t, \cdot) \int_{\Omega} u(t)\right) \tilde{u} .
$$

We claim that $B$ is well defined and is Hölder continuous in $t$. Indeed, $B(t)$ is linear in $\tilde{u}$, and 
since $X^{\alpha} \subset C^{1}(\bar{\Omega})$, we get

$$
\begin{aligned}
\|B(t) \tilde{u}\|_{L^{p}(\Omega)} \leq & \chi\left\|\nabla A^{-1} u(t)\right\|_{L^{p}(\Omega)}\|\tilde{u}\|_{C^{1}(\bar{\Omega})}+A_{0}\|\tilde{u}\|_{C^{1}(\bar{\Omega})} \\
& +\left(\chi+A_{1}+|\Omega|^{p-1} A_{2}\right)\|u(t)\|_{L^{p}(\Omega)}\|\tilde{u}\|_{C^{1}(\bar{\Omega})}+\chi\left\|A^{-1} u(t)\right\|_{L^{p}(\Omega)}\|\tilde{u}\|_{C^{1}(\bar{\Omega})} \\
\leq & C\left(1+\|u(t)\|_{C^{0}(\bar{\Omega})}\right)\|\tilde{u}\|_{X^{\alpha}} .
\end{aligned}
$$

Thus $B(t) \in \mathcal{L}\left(X^{\alpha}, L^{p}(\Omega)\right)$ and $B$ is well defined. Moreover,

$$
\begin{aligned}
& \|B(t+h) \tilde{u}-B(t) \tilde{u}\|_{L^{p}(\Omega)} \\
& \leq \chi\left\|\nabla A^{-1} u(t+h)-\nabla A^{-1} u(t)\right\|_{L^{p}(\Omega)}\|\tilde{u}\|_{C^{1}(\bar{\Omega})}+\left\|a_{0}(t+h, \cdot)-a_{0}(t, \cdot)\right\|_{C^{0}(\bar{\Omega})}\|\tilde{u}\|_{C^{1}(\bar{\Omega})} \\
& +\|u(t)\|_{L^{p}(\Omega)}\left\|a_{1}(t+h, \cdot)-a_{1}(t, \cdot)\right\|_{C^{0}(\bar{\Omega})}\|\tilde{u}\|_{C^{1}(\bar{\Omega})} \\
& +\left(\chi+A_{1}+|\Omega|^{p-1} A_{2}\right)\|u(t+h)-u(t)\|_{L^{p}(\Omega)}\|\tilde{u}\|_{C^{1}(\bar{\Omega})} \\
& +\chi\left\|A^{-1} u(t+h)-A^{-1} u(t)\right\|_{L^{p}(\Omega)}\|\tilde{u}\|_{C^{1}(\bar{\Omega})}+|\Omega|^{p-1}\|u(t)\|_{L^{p}(\Omega)}\left\|a_{2}(t+h, \cdot)-a_{2}(t, \cdot)\right\|_{C^{0}(\bar{\Omega})}\|\tilde{u}\|_{C^{1}(\bar{\Omega})} .
\end{aligned}
$$

Then by elliptic regularity, (H1), and the fact that $u(t)$ is locally Hölder continuous with respect to the $C^{0}(\bar{\Omega})$-norm, we have that $B(t)$ is Hölder continuous in $t$.

Finally, fix any $t_{1} \in\left(t_{0}, t_{0}+T_{\max }\right)$. By [11, Theorem 7.1.3], we have that

$$
\left\{\begin{array}{l}
\tilde{u}_{t}=\Delta \tilde{u}+B(t) \tilde{u}, \quad t \in\left(t_{1}, t_{0}+T_{\max }\right) \\
\tilde{u}\left(t_{1}\right)=u\left(t_{1}\right)
\end{array}\right.
$$

has a unique strong solution $\tilde{u}$ which satisfy $\tilde{u}(t) \in X^{\gamma}$ for any $\gamma<1$ and $t_{1}<t<t_{0}+T_{\max }$. By Lemma 2.3(2), $\tilde{u}$ is given by the formula

$$
\begin{aligned}
\tilde{u}(t)= & e^{-A\left(t-t_{1}\right)} u\left(t_{1}\right)-\chi \int_{t_{1}}^{t} e^{-A(t-s)} \nabla \cdot\left(\tilde{u}(s) \nabla A^{-1} u(s)\right) d s \\
& +\int_{t_{1}}^{t} e^{-A(t-s)} \tilde{u}(s)\left(1+a_{0}(s, \cdot)-a_{1}(s, \cdot) u(s)-a_{2}(s, \cdot) \int_{\Omega} u(s)\right) d s .
\end{aligned}
$$

Fix $t_{0}<t_{1}<t_{2}<t_{0}+T_{\max }$. We have by Lemma 2.2 with $\beta<\frac{1}{2}$ and $\epsilon \in\left(0, \frac{1}{2}-\beta\right)$ that

$$
\begin{aligned}
\|\tilde{u}(t)-u(t)\|_{C^{0}(\bar{\Omega})} & \leq C \int_{t_{1}}^{t}(t-s)^{-\frac{1}{2}-\beta-\epsilon}\|\tilde{u}(s)-u(s)\|_{C^{0}(\bar{\Omega})} d s+C \int_{t_{1}}^{t}(t-s)^{-\beta}\|\tilde{u}(s)-u(s)\|_{C^{0}(\bar{\Omega})} d s \\
& \leq C \int_{t_{1}}^{t}(t-s)^{-\frac{1}{2}-\beta-\epsilon}\|\tilde{u}(s)-u(s)\|_{C^{0}(\bar{\Omega})} d s
\end{aligned}
$$

for $t_{1} \leq t \leq t_{2}$ and some $C=C\left(\sup _{t_{1} \leq t \leq t_{2}}\|u(t)\|_{C^{0}(\bar{\Omega})}\right)$. Then by generalized Gronwall's inequality (see [11, page 6]), we get $\tilde{u}(t)=u(t)$ in $C^{0}(\bar{\Omega})$ on $\left[t_{1}, t_{2}\right]$. Letting $t_{1} \rightarrow t_{0}$ and $t_{2} \rightarrow t_{0}+T_{\max }$, we have $\tilde{u}(t)=u(t) \in X^{\gamma}$ for any $0 \leq \gamma<1$ and $t \in\left(t_{0}, t_{0}+T_{\max }\right)$. It then follows from Theorem 1.1(1) that $u\left(x, t ; 0, u_{0}\right):=u(t)(x)$ is a classical solution of (3.1) satisfying the properties in Theorem $1.1(2)$.

Step 3. (Nonnegativity and uniqueness) By the similar arguments as in Steps 3 and 4 in the proof of Theorem $1.1(1)$, we have that $\left(u\left(x, t ; t_{0}, u_{0}\right), v\left(x, t ; t_{0}, u_{0}\right)\right)$ is the unique nonnegative classical solution of (1.3) satisfying Theorem 1.1, where $v\left(\cdot, t ; t_{0}, u_{0}\right)=A^{-1} u\left(\cdot, t ; t_{0}, u_{0}\right)$. 
Remark 3.1. Let $\left\{t_{n}\right\} \subset \mathbb{R}$. Suppose that $\lim _{n \rightarrow \infty} a_{i}\left(t+t_{n}, x\right)=\hat{a}_{i}(t, x)$ locally uniformly in $(t, x) \in \mathbb{R} \times \bar{\Omega}$. Then $\hat{a}_{i}(t, x)(i=0,1,2)$ also satisfy the hypothesis (H1) in the introduction. Hence for any $t_{0} \in \mathbb{R}$ and $u_{0} \in X^{\alpha}$ or $u_{0} \in C^{0}(\bar{\Omega})$, (1.3) with $a_{i}(t, x)$ being replaced by $\hat{a}_{i}(t, x)$ $(i=0,1,2)$ has also a unique solution $\left(\hat{u}\left(x, t ; t_{0}, u_{0}\right), \hat{v}\left(x, t ; t_{0}, u_{0}\right)\right)$ satisfying the properties in Theorem 1.1(1) or (2).

The following corollary follows directly from Theorem 1.1 and its proof.

Corollary 3.1. (1) Let $t_{0} \in \mathbb{R}$ and $u_{0} \in X^{\alpha}$ or $C^{0}(\bar{\Omega})$ be given and let $\left(u\left(x, t ; t_{0}, u_{0}\right)\right.$, $\left.v\left(x, t ; t_{0}, u_{0}\right)\right)$ be the unique solution of (1.3) with initial condition $u\left(\cdot, t_{0} ; t_{0}, u_{0}\right)=u_{0}(\cdot)$ in Theorem 1.1(1) or (2). For any $t_{0}<t_{1}<t_{2}<t_{0}+T_{\max }$, there holds

$$
\left(u\left(x, t_{2} ; t_{0}, u_{0}\right), v\left(x, t_{2} ; t_{0}, u_{0}\right)\right)=\left(u\left(x, t_{2} ; t_{1}, u\left(\cdot, t_{1} ; t_{0}, u_{0}\right)\right), v\left(x, t_{2} ; t_{1}, u\left(\cdot, t_{1} ; t_{0}, u_{0}\right)\right) .\right.
$$

(2) Let $\left(u\left(x, t ; t_{0}, u_{0}\right), v\left(x, t ; t_{0}, u_{0}\right)\right)$ be the unique solution of (1.3) with initial condition $u\left(\cdot, t_{0} ; t_{0}, u_{0}\right)=u_{0}(\cdot) \in X$ in Theorem 1.1(1) or (2), where $X=X^{\alpha}$ or $C^{0}(\bar{\Omega})$. Then $\mathbb{R} \times X \ni\left(t_{0}, u_{0}\right) \mapsto\left(u\left(\cdot, t ; t_{0}, u_{0}\right), v\left(\cdot, t ; t_{0}, u_{0}\right)\right) \in X \times X$ is continuous locally uniformly with respect to $t \in\left(t_{0}, t_{0}+T_{\max }\right)$.

(3) Let $\left\{t_{n}\right\} \subset \mathbb{R}$. Suppose that $\lim _{n \rightarrow \infty} a_{i}\left(t+t_{n}, x\right)=\hat{a}_{i}(t, x)$ locally uniformly in $(t, x) \in$ $\mathbb{R} \times \bar{\Omega}$. For given $t_{0} \in \mathbb{R}$ and $u_{0} \in X^{\alpha}$ or $C^{0}(\bar{\Omega})$, let $\left(u_{n}\left(x, t ; t_{0}, u_{0}\right), v_{n}\left(x, t ; t_{0}, u_{0}\right)\right)$ be the solution of (1.3) with $a_{i}(t, x)$ being replaced by $a_{i}\left(t+t_{n}, x\right)(i=0,1,2)$ and with initial condition $u_{n}\left(\cdot, t_{0} ; t_{0}, u_{0}\right)=u_{0}(\cdot)$ and $\left(\hat{u}\left(x, t ; t_{0}, u_{0}\right), \hat{v}\left(x, t, ; t_{0}, u_{0}\right)\right)$ be the solution of (1.3) on $\left(t_{0}, t_{0}+\hat{T}_{\max }\right)$ with $a_{i}(t, x)$ being replaced by $\hat{a}_{i}(t, x)(i=0,1,2)$ and with initial condition $\hat{u}\left(\cdot, t_{0} ; t_{0}, u_{0}\right)=u_{0}(\cdot)$. Then for any $t \in\left(t_{0}, t_{0}+\hat{T}_{\max }\right)$,

$$
\lim _{n \rightarrow \infty}\left(u_{n}\left(\cdot, t ; t_{0}\right), v_{n}\left(\cdot, t ; t_{0}, u_{0}\right)\right)=\left(\hat{u}\left(\cdot, t ; t_{0}, u_{0}\right), \hat{v}\left(\cdot, t ; t_{0}, u_{0}\right)\right) \quad \text { in } \quad C^{0}(\bar{\Omega}) .
$$

\section{Global existence and uniform boundedness of classical solu- tions}

In this section, we investigate the global existence and the uniform boundedness of classical solutions of (1.3) with given initial functions and prove Theorem 1.2. We first prove two important lemmas.

Consider the following Lotka-Volterra Competition system of ordinary differential equations,

$$
\left\{\begin{array}{l}
\bar{u}^{\prime}=(\chi)_{+} \bar{u}(\bar{u}-\underline{u})+\bar{u}\left[a_{0, \sup }(t)-a_{1, \inf }(t) \bar{u}-|\Omega|\left(a_{2, \inf }(t)\right)_{+} \underline{u}+|\Omega|\left(a_{2, \inf }(t)\right)_{-} \bar{u}\right] \\
\underline{u}^{\prime}=(\chi)_{+} \underline{u}(\underline{u}-\bar{u})+\underline{u}\left[a_{0, \inf }(t)-a_{1, \sup }(t) \underline{u}-|\Omega|\left(a_{2, \sup }(t)\right)_{+} \bar{u}+|\Omega|\left(a_{2, \sup }(t)\right)_{-} \underline{u}\right] .
\end{array}\right.
$$

For given $u_{0} \in C^{0}(\bar{\Omega})$ with $u_{0}(x) \geq 0$ and $t_{0} \in \mathbb{R}$, let $\bar{u}_{0}=\max _{x \in \bar{\Omega}} u_{0}(x), \underline{u}_{0}=\min _{x \in \bar{\Omega}} u_{0}(x)$ and

$$
(\bar{u}(t), \underline{u}(t))=\left(\bar{u}\left(t ; t_{0}, \bar{u}_{0}, \underline{u}_{0}\right), \underline{u}\left(t ; t_{0}, \bar{u}_{0}, \underline{u}_{0}\right)\right)
$$

be the solution of (4.1) with $\left(\bar{u}\left(t_{0} ; t_{0}, \bar{u}_{0}, \underline{u}_{0}\right), \underline{u}\left(t_{0} ; t_{0}, \bar{u}_{0}, \underline{u}_{0}\right)\right)=\left(\bar{u}_{0}, \underline{u}_{0}\right)$. 
Lemma 4.1. Suppose $\inf _{t \geq t_{0}}\left\{a_{1, \mathrm{inf}}(t)-|\Omega|\left(a_{2, \mathrm{inf}}(t)\right)_{-}\right\}>(\chi)_{+}$. Then $(\bar{u}(t), \underline{u}(t))$ exists for all $t>t_{0}$ and

$$
0 \leq \underline{u}(t) \leq \bar{u}(t) \forall t \geq t_{0}
$$

Moreover, $0 \leq \bar{u}(t) \leq \max \left\{\bar{u}_{0}, \frac{a_{0, \text { sup }}}{\inf _{t \geq t_{0}}\left\{a_{1, \text { inf }}(t)-|\Omega|\left(a_{2, \text { inf }}(t)\right)_{-}-(\chi)_{+}\right\}}\right\}$.

Proof. First, note that

$$
\inf _{t \geq t_{0}}\left\{a_{1, \sup }(t)-|\Omega|\left(a_{2, \sup }(t)\right)_{-}\right\} \geq \inf _{t \in \mathbb{R}}\left\{a_{1, \inf }(t)-|\Omega|\left(a_{2, \inf }(t)\right)_{-}\right\}>(\chi)_{+} \cdot
$$

The existence of $(\bar{u}(t), \underline{u}(t))$ for all $t>t_{0}$ is then clear. For any $\epsilon>0$, let $\bar{u}_{0}^{\epsilon}=\bar{u}_{0}+\epsilon$ and $a_{0, \text { sup }}^{\epsilon}(t)=a_{0, \text { sup }}^{\epsilon}(t)+\epsilon$. Let

$$
\left(\bar{u}^{\epsilon}(t), \underline{u}^{\epsilon}(t)\right)=\left(\bar{u}^{\epsilon}\left(t ; t_{0}, \bar{u}_{0}^{\epsilon}, \underline{u}_{0}\right), \underline{u}^{\epsilon}\left(t ; t_{0}, \bar{u}_{0}^{\epsilon}, \underline{u}_{0}\right)\right),
$$

where $\left(\bar{u}^{\epsilon}\left(t ; t_{0}, \bar{u}_{0}^{\epsilon}, \underline{u}_{0}\right), \underline{u}^{\epsilon}\left(t ; t_{0}, \bar{u}_{0}^{\epsilon}, \underline{u}_{0}\right)\right)$ is the solution of (4.1) with $a_{0, \text { sup }}(t)$ being replaced by $a_{0, \text { sup }}^{\epsilon}(t)$ and $\left(\bar{u}^{\epsilon}\left(t_{0} ; t_{0}, \bar{u}_{0}^{\epsilon}, \underline{u}_{0}\right), \underline{u}^{\epsilon}\left(t_{0} ; t_{0}, \bar{u}_{0}^{\epsilon}, \underline{u}_{0}\right)\right)=\left(\bar{u}_{0}^{\epsilon}, \underline{u}_{0}\right)$. We claim that $0 \leq \underline{u}^{\epsilon}(t) \leq \bar{u}^{\epsilon}(t)$ for all $t \geq t_{0}$. Suppose by contradiction that this claim does not hold. Then since $0 \leq \underline{u}_{0}<\bar{u}_{0}^{\epsilon}$, there exist $\bar{t} \in\left(t_{0}, \infty\right)$ such that

$$
\underline{u}^{\epsilon}(t)<\bar{u}^{\epsilon}(t), \forall t \in\left[t_{0}, \bar{t}\right) \text { and } \underline{u}(\bar{t})=\bar{u}^{\epsilon}(\bar{t}) .
$$

Thus $\left(\bar{u}^{\epsilon}-\underline{u}^{\epsilon}\right)^{\prime}(\bar{t}) \leq 0$. Note that $\bar{u}^{\epsilon}(t)>0$ for $t \geq t_{0}$. Using (4.1) at $t=\bar{t}$, we get

$$
\begin{aligned}
\left(\bar{u}^{\epsilon}-\underline{u}\right)^{\prime}(\bar{t})= & \bar{u}^{\epsilon}(\bar{t})\left[a_{0, \sup }^{\epsilon}(\bar{t})-a_{0, \inf }(\bar{t})\right. \\
& \left.+\left\{a_{1, \sup }(\bar{t})-a_{1, \inf }(\bar{t})+|\Omega|\left(a_{2, \sup }(\bar{t})-a_{2, \inf }(\bar{t})\right)\right\} \bar{u}^{\epsilon}(\bar{t})\right] .
\end{aligned}
$$

It then follows that $\left(\bar{u}^{\epsilon}-\underline{u}\right)^{\prime}(\bar{t}) \geq 0$, which implies that $\left(\bar{u}^{\epsilon}-\underline{u}\right)^{\prime}(\bar{t})=0$ and then

$$
0=a_{0, \text { sup }}^{\epsilon}(\bar{t})-a_{0, \inf }(\bar{t})+\left\{a_{1, \sup }(\bar{t})-a_{1, \inf }(\bar{t})+|\Omega|\left(a_{2, \text { sup }}(\bar{t})-a_{2, \inf }(\bar{t})\right)\right\} \bar{u}^{\epsilon}(\bar{t})>0,
$$

which is a contradiction. Thus the claim holds. Letting $\epsilon \rightarrow 0$ and using continuity of solutions of (4.1) with respect to initial data and coefficients, (4.3) follows.

Furthermore, we have

$$
\begin{aligned}
\bar{u}^{\prime} & =(\chi)_{+} \bar{u}(\bar{u}-\underline{u})+\bar{u}\left[a_{0, \sup }(t)-a_{1, \inf }(t) \bar{u}-|\Omega|\left(a_{2, \inf }(t)\right)_{+} \underline{u}+|\Omega|\left(a_{2, \inf }(t)\right)_{-} \bar{u}\right] \\
& \leq \bar{u}\left[a_{0, \sup }(t)-\left\{a_{1, \inf }(t)-|\Omega|\left(a_{2, \inf }(t)\right)_{-}-(\chi)_{+}\right\} \bar{u}\right] .
\end{aligned}
$$

Thus if $\inf _{t \geq t_{0}}\left\{a_{1, \text { inf }}(t)-|\Omega|\left(a_{2, \text { inf }}(t)\right)_{-}\right\}>(\chi)_{+}$, by comparison principle, we have

$$
0<\bar{u}(t) \leq \max \left\{\bar{u}_{0}, \frac{a_{0, \text { sup }}}{\inf _{t \geq t_{0}}\left\{a_{1, \text { inf }}(t)-|\Omega|\left(a_{2, \text { inf }}(t)\right)_{-}-(\chi)_{+}\right\}}\right\} .
$$


Lemma 4.2. Suppose $\inf _{t \in \mathbb{R}}\left\{a_{1, \inf }(t)-|\Omega|\left(a_{2, \inf }(t)\right)_{-}\right\}>0$. Then

$0 \leq \int_{\Omega} u(t) \leq \max \left\{\int_{\Omega} u_{0}(x), \frac{|\Omega| a_{0, \text { sup }}}{\inf _{t \in \mathbb{R}}\left\{a_{1, \text { inf }}(t)-|\Omega|\left(a_{2, \text { inf }}(t)\right)_{-}\right\}}\right\}:=M_{0}\left(\left\|u_{0}\right\|_{L^{1}}, a_{0}, a_{1}, a_{2},|\Omega|\right)$

for all $t \in\left[t_{0}, t_{0}+T_{\max }\right)$, where $u(t)=u\left(\cdot, t ; t_{0}, u_{0}\right)$ and $u_{0} \in C^{0}(\bar{\Omega})$ with $u_{0}(x) \geq 0$.

Proof. By integrating the first equation of (1.3) over $\Omega$, we get for any $t \in\left[t_{0}, t_{0}+T_{\max }\right)$ that

$$
\begin{aligned}
\frac{d}{d t} \int_{\Omega} u(t) & =\int_{\Omega} u(t)\left\{a_{0}(t, x)-a_{1}(t, x) u(t)-a_{2}(t, x) \int_{\Omega} u(t)\right\} \\
& \leq \int_{\Omega} u(t)\left\{a_{0, \text { sup }}-a_{1, \inf }(t) u(t)-\left(a_{2, \text { inf }}(t)\right)_{+} \int_{\Omega} u(t)+\left(a_{2, \text { inf }}(t)\right)_{-} \int_{\Omega} u(t)\right\} \\
& \leq \int_{\Omega} u(t)\left\{a_{0, \text { sup }}-\frac{1}{|\Omega|}\left[a_{1, \inf }(t)-|\Omega|\left(a_{2, \text { inf }}(t)\right)_{-}\right] \int_{\Omega} u(t)\right\}
\end{aligned}
$$

Thus if $\inf _{t \in \mathbb{R}}\left\{a_{1, \text { inf }}(t)-|\Omega|\left(a_{2, \inf }(t)\right)_{-}\right\}>0$, we get by comparison principle for ODEs that

$$
0 \leq \int_{\Omega} u(t) \leq \max \left\{\int_{\Omega} u_{0}(x), \frac{|\Omega| a_{0, \text { sup }}}{\inf _{t \in \mathbb{R}}\left\{a_{1, \text { inf }}(t)-|\Omega|\left(a_{2, \text { inf }}(t)\right)_{-}\right\}}\right\}
$$

We now prove Theorem 1.2 for $\chi>0$ and the proof for the case $\chi \leq 0$ follows from similar arguments with proper adaptations.

Proof of Theorem 1.2. (1) Let $(\bar{u}(t), \underline{u}(t))$ be as in (4.2). It suffices to prove that $0 \leq \underline{u}(t) \leq$ $u\left(x, t ; t_{0}, u_{0}\right) \leq \bar{u}(t)$ for all $t_{0} \leq t<t_{0}+T_{\max }$ and $x \in \bar{\Omega}$.

Observe that for any $\epsilon>0$, there exists $t_{0}<t_{\epsilon}<t_{0}+T_{\max }$ such that

$$
\underline{u}(t)-2 \epsilon<u\left(x, t ; t_{0}, u_{0}\right)<\bar{u}(t)+2 \epsilon, \quad \text { for all }(x, t) \in \Omega \times\left[t_{0}, t_{\epsilon}\right) .
$$

Let

$$
T_{\epsilon}=\sup \left\{t_{\epsilon} \in\left(t_{0}, t_{0}+T_{\max }\right) \mid \underline{u}(t)-2 \epsilon<u\left(x, t ; t_{0}, u_{0}\right)<\bar{u}(t)+2 \epsilon \quad \forall(x, t) \in \Omega \times\left[t_{0}, t_{\epsilon}\right)\right\} .
$$

It then suffices to prove that $T_{\epsilon}=t_{0}+T_{\max }$.

Assume by contradiction that $T_{\epsilon}<t_{0}+T_{\max }$. Then there is $x_{0} \in \bar{\Omega}$ such that

$$
\text { either } u\left(x_{0}, T_{\epsilon} ; t_{0}, u_{0}\right)=\underline{u}\left(T_{\epsilon}\right)-2 \epsilon \text { or } u\left(x_{0}, T_{\epsilon} ; t_{0}, u_{0}\right)=\bar{u}\left(T_{\epsilon}\right)+2 \epsilon .
$$

Let $\bar{U}(x, t)=u\left(x, t ; t_{0}, u_{0}\right)-\bar{u}(t)$ and $\underline{U}(x, t)=u\left(x, t ; t_{0}, u_{0}\right)-\underline{u}(t)$.

Note that for $t \in\left(t_{0}, t_{0}+T_{\max }\right), \bar{U}$ satisfies

$$
\begin{aligned}
\bar{U}_{t}-\Delta \bar{U} \leq & -\chi \nabla \bar{U} \cdot \nabla v+\bar{U}\left[a_{0, \sup }(t)-\left(a_{1, \inf }(t)-\chi\right)(u+\bar{u})-\chi \underline{u}\right] \\
& -\chi u(v-\underline{u})-a_{2, \inf }(t)\left(\int_{\Omega} u\right) u+|\Omega|\left(a_{2, \inf }(t)\right)_{+} \underline{u} \bar{u}-|\Omega|\left(a_{2, \inf }(t)\right)_{-} \bar{u}^{2} .
\end{aligned}
$$

By $-a_{2, \inf }(t)\left(\int_{\Omega} u\right) u=-\left(a_{2, \inf }(t)\right)_{+}\left(\int_{\Omega} u\right) u+\left(a_{2, \text { inf }}(t)\right)_{-}\left(\int_{\Omega} u\right) u$, we get for $t \in\left(t_{0}, t_{0}+T_{\max }\right)$ that 


$$
\begin{aligned}
\bar{U}_{t}-\Delta \bar{U} \leq & -\chi \nabla \bar{U} \cdot \nabla v+\bar{U}\left[a_{0, \sup }(t)-\left(a_{1, \inf }(t)-\chi\right)(u+\bar{u})-\chi \underline{u}\right] \\
& -\chi u(v-\underline{u})-\left(a_{2, \inf }(t)\right)_{+}\left(\left(\int_{\Omega} u\right) u-|\Omega| \underline{u} \bar{u}\right)+\left(a_{2, \inf }(t)\right)_{-}\left(\int_{\Omega} u\right) u-|\Omega|\left(a_{2, \inf }(t)\right)_{-} \bar{u}^{2} \\
& \leq-\chi \nabla \bar{U} \cdot \nabla v+\bar{U}\left[a_{0, \sup }(t)-\left(a_{1, \inf }(t)-\chi\right)(u+\bar{u})-\chi \underline{u}\right] \\
& -\chi u(v-\underline{u})-\left(a_{2, \inf }(t)\right)_{+}\left(\left(\int_{\Omega} u\right) u-|\Omega| \underline{u} \bar{u}\right) \\
& +\left(a_{2, \inf }(t)\right)_{-}\left(u \int_{\Omega}(u-\bar{u})\right)-|\Omega|\left(a_{2, \inf }(t)\right)_{-} \bar{u}(\bar{u}-u) \\
& \leq-\chi \nabla \bar{U} \cdot \nabla v+\bar{U}\left[a_{0, \sup }(t)-\left(a_{1, \inf }(t)-\chi\right)(u+\bar{u})-\chi \underline{u}+|\Omega|\left(a_{2, \inf }(t)\right)_{-} \bar{u}\right] \\
& -\chi u(v-\underline{u})-\left(a_{2, \inf }(t)\right)_{+}\left(\left(\int_{\Omega} u\right) u-|\Omega| \underline{u} \bar{u}\right)+\left(a_{2, \inf }(t)\right)_{-}\left(\int_{\Omega} \bar{U}\right) u .
\end{aligned}
$$

We claim that $\int_{\Omega} \bar{U}_{+}^{2}(x, t) d x$ is weakly differentiable in $t$ and moreover

$$
\frac{d}{d t} \int_{\Omega} \bar{U}_{+}^{2}(x, t) d x=2 \int_{\Omega} \bar{U}_{+}(x, t) \bar{U}_{t}(x, t) d x \quad \text { for } \quad \text { a.e. } t \in\left(t_{0}, t_{0}+T_{\max }\right),
$$

and

$$
\int_{\Omega} \bar{U}_{+}^{2}(x, t) d x=\int_{\Omega} \bar{U}_{+}^{2}\left(x, t_{0}\right) d x+\int_{t_{0}}^{t}\left(\frac{d}{d t} \int_{\Omega} \bar{U}_{+}^{2}(x, \tau) d x\right) d \tau \quad \forall t \in\left(t_{0}, t_{0}+T_{\max }\right) .
$$

In order to prove the claim we define for $r>0$,

$$
F_{r}(z)= \begin{cases}\left(z^{2}+r\right)^{\frac{1}{2}}-r, & \text { if } z>0 \\ 0, & \text { if } z \leq 0\end{cases}
$$

Then $F_{r} \in C^{1}(\mathbb{R})$,

$$
F_{r}^{\prime}(z)= \begin{cases}z\left(z^{2}+r\right)^{-\frac{1}{2}}, & \text { if } z>0 \\ 0, & \text { if } z \leq 0\end{cases}
$$

Note that $\left|F_{r}{ }^{\prime}\right| \leq 1$ and that we have the following pointwise convergence,

$$
\bar{U}_{+}(x, t)=\lim _{r \rightarrow 0} F_{r}(\bar{U}(x, t)) .
$$

This implies that

$$
\int_{\Omega} \bar{U}_{+}^{2}(x, t) d x=\lim _{r \rightarrow 0} \int_{\Omega} F_{r}^{2}(\bar{U}(x, t)) d x \quad \forall t \in\left(t_{0}, t_{0}+T_{\max }\right) .
$$

Note also that $\int_{\Omega} F_{r}^{2}(\bar{U}(x, t)) d x$ is differentiable in $t$ and

$$
\frac{d}{d t} \int_{\Omega} F_{r}^{2}(\bar{U}(x, t)) d x=2 \int_{\Omega}\left(\left(\bar{U}_{+}^{2}(x, t)+r\right)^{\frac{1}{2}}-r\right) \bar{U}_{+}(x, t)\left(\bar{U}_{+}^{2}(x, t)+r\right)^{-\frac{1}{2}} \bar{U}_{t}(x, t) d x .
$$

By (4.9), for any $\delta>0$, there is $M_{\delta}>0$ such that for any $r>0$

$$
\left|\int_{\Omega} F_{r}^{2}\left(\bar{U}\left(x, t_{1}\right)\right) d x-\int_{\Omega} F_{r}^{2}\left(\bar{U}\left(x, t_{2}\right)\right) d x\right| \leq M_{\delta}\left|t_{1}-t_{2}\right| \quad \forall t_{1}, t_{2} \in\left[t_{0}+\delta, t_{0}+T_{\max }-\delta\right] .
$$


Then by (4.8) and (4.10), we have

$$
\left|\int_{\Omega} \bar{U}_{+}^{2}\left(x, t_{1}\right) d x-\int_{\Omega} \bar{U}_{+}^{2}\left(x, t_{2}\right) d x\right| \leq M_{\delta}\left|t_{1}-t_{2}\right| \quad \forall t_{1}, t_{2} \in\left[t_{0}+\delta, t_{0}+T_{\max }-\delta\right] .
$$

Let $\phi \in C_{c}^{\infty}\left(\left(t_{0}, t_{0}+T_{\max }\right)\right)$. We have by integration by part that

$$
\int_{t_{0}}^{T_{\max }} \frac{d}{d t}\left(\int_{\Omega} F_{r}(\bar{U}(x, t))^{2} d x\right) \phi(t) d t=-\int_{t_{0}}^{T_{\max }}\left(\int_{\Omega} F_{r}(\bar{U}(x, t))^{2} d x\right) \phi_{t}(t) d t .
$$

By Lebesgue Dominated Theorem we get from (4.8) that

$$
\lim _{r \rightarrow 0}\left(-\int_{t_{0}}^{T_{\max }}\left(\int_{\Omega} F_{r}(\bar{U}(x, t))^{2} d x\right) \phi_{t}(t) d t\right)=-\int_{t_{0}}^{T_{\max }} \int_{\Omega}\left(\bar{U}_{+}(x, t)\right)^{2} d x \phi_{t}(t) d t,
$$

and from (4.9) that

$$
\lim _{r \rightarrow 0} \int_{t_{0}}^{T_{\max }} \frac{d}{d t}\left(\int_{\Omega} F_{r}(\bar{U}(x, t))^{2} d x\right) \phi(t) d t=2 \int_{t_{0}}^{T_{\max }} \int_{\Omega} \bar{U}_{+}(x, t) \bar{U}_{t}(x, t) d x \phi(t) d t .
$$

Thus it follows from equations (4.12) that

$$
\int_{t_{0}}^{T_{\max }} \int_{\Omega}\left(\bar{U}_{+}(x, t)\right)^{2} d x \phi_{t}(t) d t=-2 \int_{t_{0}}^{T_{\max }} \int_{\Omega} \bar{U}_{+}(x, t) \bar{U}_{t}(x, t) d x \phi(t) d t .
$$

This implies that $\int_{\Omega} \bar{U}_{+}^{2}(x, t) d x$ is weakly differentiable and (4.6) holds. By (4.6), (4.11), and the Fundamental Theorem of Calculus for Lebesgue Integrals, we have for any $t, t_{1} \in\left(t_{0}, t_{0}+T_{\max }\right)$ that

$$
\int_{\Omega} \bar{U}_{+}^{2}(x, t) d x=\int_{\Omega} \bar{U}_{+}^{2}\left(x, t_{1}\right) d x+\int_{t_{1}}^{t}\left(\frac{d}{d t} \int_{\Omega} \bar{U}_{+}^{2}(x, \tau) d x\right) d \tau .
$$

Letting $t_{1} \rightarrow t_{0}$, (4.7) follows.

By (4.6), multiplying (4.5) by $\bar{U}_{+}$and integrating with respect to $x$ over $\Omega$, we get

$$
\begin{aligned}
& \frac{1}{2} \frac{d}{d t} \int_{\Omega}\left(\bar{U}_{+}\right)^{2}+\int_{\Omega}\left|\nabla\left(\bar{U}_{+}\right)\right|^{2} \\
& \leq \int_{\Omega}\left(\bar{U}_{+}\right)^{2}\left[a_{0, \sup }(t)+\chi \frac{1}{2} u-\frac{1}{2} \chi v-\left(a_{1, \inf }(t)-\chi\right)(u+\bar{u})-\chi \underline{u}+|\Omega|\left(a_{2, \inf }(t)\right)_{-} \bar{u}\right] \\
& \quad-\chi \int_{\Omega}\left(\bar{U}_{+}\right) u(v-\underline{u})-\left(a_{2, \inf }(t)\right)_{+} \int_{\Omega}\left(\bar{U}_{+}\right)\left[\left(\int_{\Omega} u\right) u-|\Omega| \underline{u} \bar{u}\right]+\left(a_{2, \inf }(t)\right)_{-} \int_{\Omega} \bar{U}_{+}\left(u \int_{\Omega} \bar{U}\right)
\end{aligned}
$$

for a.e. $t \in\left(t_{0}, t_{0}+T_{\max }\right)$. Note that

$$
\left(a_{2, \inf }(t)\right)_{-} \int_{\Omega} \bar{U}_{+}\left(u \int_{\Omega} \bar{U}\right) \leq\left(a_{2, \inf }(t)\right)_{-} \int_{\Omega} \bar{U}_{+} u\left(\int_{\Omega} \bar{U}_{+}\right) \leq|\Omega|\left(a_{2, \inf }(t)\right)_{-}(\bar{u}+2 \epsilon) \int_{\Omega} \bar{U}_{+}^{2}
$$

and

$$
\begin{aligned}
-\left(a_{2, \text { inf }}(t)\right)_{+} \int_{\Omega} \bar{U}_{+}\left(\left(\int_{\Omega} u\right) u-|\Omega| \underline{u} \bar{u}\right) & =-\left(a_{2, \inf }(t)\right)_{+} \int_{\Omega} \bar{U}_{+}^{2}\left(\int_{\Omega} u\right)-\left(a_{2, \inf }(t)\right)_{+} \int_{\Omega} \bar{U}_{+} \bar{u} \int_{\Omega} \underline{U} \\
& \leq-\left(a_{2, \inf }(t)\right)_{+} \int_{\Omega} \bar{U}_{+} \bar{u} \int_{\Omega} \underline{U} \leq\left(a_{2, \inf }(t)\right)_{+} \bar{u} \int_{\Omega} \bar{U}_{+} \int_{\Omega} \underline{U}_{-} \\
& \leq|\Omega|\left(a_{2, \mathrm{inf}}(t)\right)_{+} \frac{\bar{u}}{2}\left(\int_{\Omega} \bar{U}_{+}^{2}+\int_{\Omega} \underline{U}_{-}^{2}\right) .
\end{aligned}
$$


Moreover by using the second equation of (1.3), we get

$$
\int_{\Omega}\left|\nabla(v-\underline{u})_{-}\right|^{2}+\int_{\Omega}(v-\underline{u})_{-}^{2}=-\int_{\Omega}(\underline{U})(v-\underline{u})_{-} \leq \int_{\Omega}(\underline{U})_{-}(v-\underline{u})_{-} .
$$

Thus by Young's inequality, we have $\int_{\Omega}(v-\underline{u})_{-}^{2} \leq \int_{\Omega}\left(\underline{U}_{-}\right)^{2}$. Therefore

$$
-\chi \int_{\Omega}\left(\bar{U}_{+}\right) u(v-\underline{u}) \leq \frac{\chi(\bar{u}+2 \epsilon)}{2}\left(\int_{\Omega}\left(\bar{U}_{+}\right)^{2}+\int_{\Omega}\left(\underline{U}_{-}\right)^{2}\right) .
$$

Combining all these inequalities, we get

$$
\begin{aligned}
& \frac{1}{2} \frac{d}{d t} \int_{\Omega}\left(\bar{U}_{+}\right)^{2}+\int_{\Omega}\left|\nabla\left(\bar{U}_{+}\right)\right|^{2} \\
& \leq \int_{\Omega}\left(\bar{U}_{+}\right)^{2}\left[a_{0, \sup }(t)+\chi \frac{1}{2} u+2|\Omega|\left(a_{2, \inf }(t)\right)_{-}(\bar{u}+\epsilon)+|\Omega|\left(a_{2, \inf }(t)\right)_{+} \frac{\bar{u}}{2}+\frac{\chi(\bar{u}+2 \epsilon)}{2}\right] \\
& +\left[\frac{\chi(\bar{u}+2 \epsilon)}{2}+|\Omega|\left(a_{2, \inf }(t)\right)_{+} \frac{\bar{u}}{2}\right] \int_{\Omega}\left(\underline{U}_{-}\right)^{2} \\
& \leq \int_{\Omega}\left(\bar{U}_{+}\right)^{2}\left[a_{0, \sup }(t)+\left(2|\Omega|\left(a_{2, \inf }(t)\right)_{-}+\chi\right)(\bar{u}+2 \epsilon)+|\Omega|\left(a_{2, \inf }(t)\right)_{+} \frac{\bar{u}}{2}\right] \\
& +\left[\frac{\chi(\bar{u}+2 \epsilon)}{2}+|\Omega|\left(a_{2, \text { inf }}(t)\right)_{+} \frac{\bar{u}}{2}\right] \int_{\Omega}\left(\underline{U}_{-}\right)^{2} \quad \text { for a.e. } t \in\left(t_{0}, T_{\epsilon}\right] .
\end{aligned}
$$

Similarly, we have that $\int_{\Omega} \underline{U}_{-}^{2}(x, t) d x$ is weakly differentiable in $t$ and moreover

$$
\frac{d}{d t} \int_{\Omega} \underline{U}_{-}^{2}(x, t) d x=2 \int_{\Omega} \underline{U}_{-}(x, t) \underline{U}_{t}(x, t) d x \quad \text { for } \quad \text { a.e. } t \in\left(t_{0}, t_{0}+T_{\max }\right),
$$

and

$$
\int_{\Omega} \underline{U}_{-}^{2}(x, t) d x=\int_{\Omega} \underline{U}_{-}^{2}\left(x, t_{0}\right) d x+\int_{t_{0}}^{t}\left(\frac{d}{d t} \int_{\Omega} \underline{U}_{-}^{2}(x, \tau) d x\right) d \tau \quad \forall t \in\left(t_{0}, t_{0}+T_{\max }\right) .
$$

Also we have

$$
\begin{aligned}
\underline{U}_{t}-\Delta \underline{U} \geq & -\chi \nabla \bar{U} \cdot \nabla v+\bar{U}\left[a_{0, \inf }(t)-\left(a_{1, \sup }(t)-\chi\right)(u+\underline{u})-\chi \bar{u}+|\Omega|\left(a_{2, \sup }(t)\right)_{-} \underline{u}\right] \\
& -\chi u(v-\bar{u})-\left(a_{2, \sup }(t)\right)_{+}\left(\left(\int_{\Omega} u\right) u-|\Omega| \underline{u} \bar{u}\right)+\left(a_{2, \sup }(t)\right)_{-}\left(\int_{\Omega} \underline{U}\right) u .
\end{aligned}
$$

By multiplying the above inequality by $\underline{-}_{-}$- and integrating with respect to $x$ over $\Omega$, we have

$$
\begin{aligned}
& \frac{1}{2} \frac{d}{d t} \int_{\Omega}\left(\underline{U}_{-}^{2}\right)+\int_{\Omega}\left|\nabla\left(\underline{U}_{-}\right)\right|^{2} \\
& \leq \int_{\Omega}\left(\underline{U}_{-}\right)^{2}\left[a_{0, \inf }(t)+\left(2|\Omega|\left(a_{2, \sup }(t)\right)_{-}+\chi\right)(\bar{u}+2 \epsilon)+|\Omega|\left(a_{2, \sup }(t)\right)_{+} \frac{\underline{u}}{2}\right] \\
& \quad+\left[\frac{\chi(\bar{u}+2 \epsilon)}{2}+|\Omega|\left(a_{2, \text { sup }}(t)\right)_{+} \frac{u}{\overline{2}}\right] \int_{\Omega}\left(\bar{U}_{+}\right)^{2} \quad \text { for } \quad \text { a.e. } t \in\left(t_{0}, T_{\epsilon}\right] .
\end{aligned}
$$


By (4.7), (4.14), (4.16), and (4.17), we have

$$
\begin{aligned}
& \frac{1}{2} \int_{\Omega}\left(\bar{U}_{+}^{2}(x, t)+\underline{U}_{-}^{2}(x, t)\right) d x \\
& \leq \frac{1}{2} \int_{\Omega}\left(\bar{U}_{+}^{2}\left(x, t_{0}\right)+\underline{U}_{-}^{2}\left(x, t_{0}\right)\right) d x \\
& +\int_{t_{0}}^{t} \int_{\Omega}\left(\bar{U}_{+}^{2}(x, \tau)+\underline{U}_{-}^{2}(x, \tau)\right)\left[a_{0, \sup }(\tau)+\left(2|\Omega|\left(a_{2, \inf }(\tau)\right)_{-}+\chi\right)(\bar{u}+2 \epsilon)+|\Omega|\left(a_{2, \text { sup }}(\tau)\right)_{+} \frac{\bar{u}}{2}\right] d \tau \\
& +\int_{t_{0}}^{t}\left[\frac{\chi(\bar{u}+2 \epsilon)}{2}+|\Omega|\left(a_{2, \sup }(\tau)\right)_{+} \frac{\bar{u}}{2}\right] \int_{\Omega}\left(\bar{U}_{+}^{2}(x, \tau)+\underline{U}_{-}^{2}(x, \tau)\right) d \tau \quad \forall t \in\left(t_{0}, T_{\epsilon}\right]
\end{aligned}
$$

This together with $\bar{U}_{+}\left(\cdot, t_{0}\right)=\underline{U}_{-}\left(\cdot, t_{0}\right)=0$ and Gronwall's inequality implies $\bar{U}_{+}(x, t)=$ $\underline{U}_{-}(x, t)=0$ for $(x, t) \in \Omega \times\left[t_{0}, T_{\epsilon}\right]$. Therefore,

$$
\underline{u}(t) \leq u\left(x, t ; t_{0}, u_{0}\right) \leq \bar{u}(t) \quad(x, t) \in \bar{\Omega} \times\left[t_{0}, T_{\epsilon}\right] .
$$

This is a contradiction. Therefore, $T_{\epsilon}=t_{0}+T_{\max }$. We then have $T_{\max }=\infty$ and (1.13) holds.

(2) We divide the proof in three steps. Note that the statements in these steps have already been establish in the case of constant coefficients and $a_{2}=0$, by Tello and Winkler in [43, Lemma 2.2, 2.3 and 2.4]. For simplicity in notation, we denote $\left(u\left(\cdot, t ; t_{0}, u_{0}\right), v\left(\cdot, t ; t_{0}, u_{0}\right)\right)$ by $(u(t), v(t))$.

Step 1. In this step, we prove that for any $\gamma \in\left(1, \frac{\chi}{\left(\chi-a_{1, \text { inf }}\right)_{+}}\right)$, there is $C=C\left(\gamma,\left\|u_{0}\right\|_{L^{\gamma}}\right.$, $\left.\left\|u_{0}\right\|_{L^{1}}, A_{0}, A_{2}, a_{1},|\Omega|\right)$ such that

$$
\int_{\Omega} u^{\gamma}(t) \leq C \quad \forall t \in\left[t_{0}, t_{0}+T_{\max }\right)
$$

and

$$
\int_{t_{0}}^{T} \int_{\Omega} u^{\gamma+1}(t)+\int_{t_{0}}^{T} \int_{\Omega}\left|\nabla u^{\frac{\gamma}{2}}(t)\right|^{2} \leq \widetilde{C}(T+1) \quad \forall T \in\left(t_{0}, t_{0}+T_{\max }\right) .
$$

By multiplying the first equation of (1.3) by $u^{\gamma-1}(t)$ and integrating with respect to $x$ over $\Omega$, we have for $t \in\left(t_{0}, t_{0}+T_{\max }\right)$ that

$$
\begin{aligned}
\frac{1}{\gamma} \frac{d}{d t} \int_{\Omega} u^{\gamma}(t)+\frac{4(\gamma-1)}{\gamma^{2}} \int_{\Omega}\left|\nabla u^{\frac{\gamma}{2}}(t)\right|^{2}= & (\gamma-1) \chi \int_{\Omega} u^{\gamma-1}(t) \nabla u(t) \cdot \nabla v(t) \\
& +\int_{\Omega} u^{\gamma}(t)\left[a_{0}(t, \cdot)-a_{1}(t, \cdot) u(t)-a_{2}(t, \cdot) \int_{\Omega} u(t)\right] .
\end{aligned}
$$

By multiplying the second equation of (1.3) by $u^{\gamma}(\cdot)$ and integrating over $\Omega$, we get

$$
(\gamma-1) \chi \int_{\Omega} u^{\gamma-1}(t) \nabla u(t) \cdot \nabla v(t)=-\frac{\chi(\gamma-1)}{\gamma} \int_{\Omega} v(t) u^{\gamma}(t)+\frac{\chi(\gamma-1)}{\gamma} \int_{\Omega} u^{\gamma+1}(t) .
$$

Thus we have for $t \in\left(t_{0}, t_{0}+T_{\max }\right)$ that

$$
\begin{aligned}
& \frac{1}{\gamma} \frac{d}{d t} \int_{\Omega} u^{\gamma}(t)+\frac{4(\gamma-1)}{\gamma^{2}} \int_{\Omega}\left|\nabla u^{\frac{\gamma}{2}}(t)\right|^{2} \\
& =-\frac{\chi(\gamma-1)}{\gamma} \int_{\Omega} v u^{\gamma}(t)+\frac{\chi(\gamma-1)}{\gamma} \int_{\Omega} u^{\gamma+1}(t)+\int_{\Omega} u^{\gamma}(t)\left[a_{0}(t, \cdot)-a_{1}(t, \cdot) u(t)-a_{2}(t, \cdot) \int_{\Omega} u(t)\right] .
\end{aligned}
$$


By Lemma 4.2, we have $\left(a_{2, \text { inf }}(t)\right)_{-} \int_{\Omega} u(t) \leq A_{2} M_{0}$. Therefore

$$
\begin{aligned}
& \frac{1}{\gamma} \frac{d}{d t} \int_{\Omega} u^{\gamma}(t)+\frac{4(\gamma-1)}{\gamma^{2}} \int_{\Omega}\left|\nabla u^{\frac{\gamma}{2}}(t)\right|^{2} \\
& \leq \frac{\chi(\gamma-1)}{\gamma} \int_{\Omega} u^{\gamma+1}(t)+\int_{\Omega} u^{\gamma}(t)\left[a_{0}(t, \cdot)-a_{1, \inf } u(t)+\left(a_{2, \inf }(t)\right)_{-} \int_{\Omega} u(t)\right] \\
& \leq \frac{\chi(\gamma-1)}{\gamma} \int_{\Omega} u^{\gamma+1}(t)+\int_{\Omega} u^{\gamma}(t)\left[A_{0}+A_{2} M_{0}-a_{1, \mathrm{inf}} u(t)\right] \\
& \leq-\left[a_{1, \mathrm{inf}}-\frac{\chi(\gamma-1)}{\gamma}\right] \int_{\Omega} u^{\gamma+1}(t)+\left(A_{0}+A_{2} M_{0}\right) \int_{\Omega} u^{\gamma}(t) .
\end{aligned}
$$

Note that $\mu:=a_{1, \text { inf }}-\frac{\chi(\gamma-1)}{\gamma}>0$. By Young's inequality, we have

$$
\left(A_{0}+A_{2} M_{0}\right) \int_{\Omega} u^{\gamma}(t) \leq \frac{1}{2} \mu \int_{\Omega} u^{\gamma+1}(t)+C\left(\gamma, A_{0}, A_{2}, a_{1},\left\|u_{0}\right\|_{L^{1}},|\Omega|\right) .
$$

Thus

$$
\frac{1}{\gamma} \frac{d}{d t} \int_{\Omega} u^{\gamma}(t)+\frac{4(\gamma-1)}{\gamma^{2}} \int_{\Omega}\left|\nabla u^{\frac{\gamma}{2}}(t)\right|^{2} \leq-\frac{\mu}{2} \int_{\Omega} u^{\gamma+1}(t)+C\left(\gamma, A_{0}, A_{2}, a_{1},\left\|u_{0}\right\|_{L^{1}},|\Omega|\right) .
$$

This together with Hölder's inequality implies that

$$
\frac{d}{d t} \int_{\Omega} u^{\gamma}(t) \leq-\frac{\mu \gamma}{|\Omega|^{\frac{1}{\gamma}}}\left(\int_{\Omega} u^{\gamma}(t)\right)^{\frac{\gamma+1}{\gamma}}+C\left(\gamma, A_{0}, A_{2}, a_{1},\left\|u_{0}\right\|_{L^{1}},|\Omega|\right) .
$$

It then follows that

$$
\int_{\Omega} u^{\gamma}(t) \leq \max \left\{\int_{\Omega} u_{0}^{\gamma},\left(\frac{C\left(\gamma, A_{0}, A_{2}, a_{1},\left\|u_{0}\right\|_{L^{1}},|\Omega|\right)}{\mu}\right)^{\frac{\gamma}{\gamma+1}}\right\} \quad \forall t \in\left[t_{0}, t_{0}+T_{\max }\right) .
$$

Now by integrating (4.21) on $\left(t_{0}, T\right)$, we get

$$
\int_{t_{0}}^{T} \int_{\Omega} u^{\gamma+1}(t)+\int_{t_{0}}^{T} \int_{\Omega}\left|\nabla u^{\frac{\gamma}{2}}(t)\right|^{2} \leq \widetilde{C}(T+1) .
$$

(4.18) and (4.19) then follow.

Step 2. In this step, we prove that for any $\gamma>1$, there is $C=C\left(\gamma,\left\|u_{0}\right\|_{L^{\gamma}},\left\|u_{0}\right\|_{L^{1}}, A_{0}, A_{2}, a_{1},|\Omega|\right)$ such that

$$
\int_{\Omega} u^{\gamma}(t) \leq C \quad \forall t \in\left[t_{0}, t_{0}+T_{\max }\right)
$$

Since $a_{1, \text { inf }}>\frac{\chi(n-2)}{n}$, we get $\frac{n}{2}<\frac{\chi}{\left(\chi-a_{1, \text { inf })_{+}}\right.}$. Choose $\gamma_{0} \in\left(\frac{n}{2}, \frac{\chi}{\left(\chi-a_{1, \text { inf }}\right)_{+}}\right)$, then by (4.18), we have

$$
\int_{\Omega} u^{\gamma_{0}}(t) \leq C=C\left(\gamma_{0},\left\|u_{0}\right\|_{L^{\gamma_{0}}},\left\|u_{0}\right\|_{L^{1}}, A_{0}, A_{2}, a_{1},|\Omega|\right) .
$$

Let $\gamma>1$. If $1<\gamma \leq \gamma_{0}$, the result follows by the continuous inclusion $L^{\gamma_{0}}(\Omega) \subset L^{\gamma}(\Omega)$. Suppose $\gamma>\gamma_{0}$. Let $\tilde{\mu}=2\left|\left(a_{1, \text { inf }}-\frac{\chi(\gamma-1)}{\gamma}\right)\right|+1>0$. By Young's inequality we get

$$
\left(A_{0}+A_{2} M_{0}\right) \int_{\Omega} u^{\gamma}(t) \leq \frac{\tilde{\mu}}{2} \int_{\Omega} u^{\gamma+1}(t)+C\left(\gamma, A_{0}, A_{2}, a_{1},\left\|u_{0}\right\|_{L^{1}},|\Omega|\right) .
$$


This together with (4.20) implies that

$$
\frac{1}{\gamma} \frac{d}{d t} \int_{\Omega} u^{\gamma}(t)+\frac{4(\gamma-1)}{\gamma^{2}} \int_{\Omega}\left|\nabla u^{\frac{\gamma}{2}}(t)\right|^{2} \leq \tilde{\mu} \int_{\Omega} u^{\gamma+1}(t)+C\left(\gamma, A_{0}, A_{2}, a_{1},\left\|u_{0}\right\|_{L^{1}},|\Omega|\right) .
$$

Note that

$$
\int_{\Omega} u^{\gamma_{0}}(t)=\left\|u^{\frac{\gamma}{2}}(t)\right\|_{L^{\frac{2 \gamma_{0}}{\gamma}}}^{\frac{2 \gamma_{0}}{\gamma}} \leq C \quad \text { and } \quad \int_{\Omega} u^{\gamma+1}(t)=\left\|u^{\frac{\gamma}{2}}(t)\right\|_{L^{\frac{2(\gamma+1)}{\gamma}}}^{\frac{2(\gamma+1)}{\gamma}} .
$$

By Gagliardo-Nirenberg inequality, there exists $C_{0}$ depending on the domain $\Omega$ and $\gamma$ such that

$$
\begin{aligned}
\int_{\Omega} u^{\gamma+1}(t)=\left\|u^{\frac{\gamma}{2}}(t)\right\|_{L^{\frac{2(\gamma+1)}{\gamma}}}^{\frac{2(\gamma+1)}{\gamma}} & \leq C_{0}\left\|\nabla u^{\frac{\gamma}{2}}(t)\right\|_{L^{2}}^{\frac{2(\gamma+1) a}{\gamma}}\left\|u^{\frac{\gamma}{2}}(t)\right\|_{L^{\frac{2 \gamma_{0}}{\gamma}}}^{\frac{2(\gamma+1)(1-a)}{\gamma}}+C_{0}\left\|u^{\frac{\gamma}{2}}(t)\right\|_{L^{\frac{2 \gamma_{0}}{\gamma}}}^{\frac{2 \gamma_{0}}{\gamma}} \\
& \leq C\left(\gamma,\left\|u_{0}\right\|_{L^{\gamma}},\left\|u_{0}\right\|_{L^{1}}, A_{0}, A_{2}, a_{1},|\Omega|\right)\left(\left\|\nabla u^{\frac{\gamma}{2}}(t)\right\|_{L^{2}}^{\frac{2(\gamma+1) a}{\gamma}}+1\right),
\end{aligned}
$$

where $a=\frac{\frac{n \gamma}{22}-\frac{n \gamma}{2(\gamma+1)}}{1+\frac{n}{2}\left(\frac{\gamma}{\gamma_{0}}-1\right)}$. Since $\frac{n}{2}<\gamma_{0}<\gamma$, we have $0<a<1$ and $2 \frac{(\gamma+1)}{\gamma} a-2=-\frac{2-\frac{n}{\gamma_{0}}}{1+\frac{n}{2}\left(\frac{\gamma}{\gamma_{0}}-1\right)}<0$. By applying Young's Inequality, we get for any $\epsilon>0$

$$
\begin{aligned}
& C\left(\gamma,\left\|u_{0}\right\|_{L^{\gamma}},\left\|u_{0}\right\|_{L^{1}}, A_{0}, A_{2}, a_{1},|\Omega|\right)\left\|\nabla u^{\frac{\gamma}{2}}(t)\right\|_{L^{2}}^{\frac{2(\gamma+1) a}{\gamma}} \\
& \leq \epsilon\left\|\nabla u^{\frac{\gamma}{2}}(t)\right\|_{L^{2}}^{2}+C\left(\epsilon, \gamma,\left\|u_{0}\right\|_{L^{\gamma}},\left\|u_{0}\right\|_{L^{1}}, A_{0}, A_{2}, a_{1},|\Omega|\right) .
\end{aligned}
$$

Therefore

$$
\int_{\Omega} u^{\gamma+1}(t) \leq \epsilon\left\|\nabla u^{\frac{\gamma}{2}}(t)\right\|_{L^{2}}^{2}+C\left(\epsilon, \gamma,\left\|u_{0}\right\|_{L^{\gamma}},\left\|u_{0}\right\|_{L^{1}}, A_{0}, A_{2}, a_{1},|\Omega|\right)
$$

and then

$$
-\frac{4(\gamma-1)}{\gamma^{2}} \int_{\Omega}\left|\nabla u^{\frac{\gamma}{2}}(t)\right|^{2} \leq-\frac{4(\gamma-1)}{\epsilon \gamma^{2}} \int_{\Omega} u^{\gamma+1}(t)+\frac{4(\gamma-1)}{\epsilon \gamma^{2}} C\left(\epsilon, \gamma,\left\|u_{0}\right\|_{L^{\gamma}},\left\|u_{0}\right\|_{L^{1}}, A_{0}, A_{2}, a_{1},|\Omega|\right) .
$$

It then follows that

$$
\frac{1}{\gamma} \frac{d}{d t} \int_{\Omega} u^{\gamma}(t) \leq-\left(\frac{4(\gamma-1)}{\epsilon \gamma^{2}}-\tilde{\mu}\right) \int_{\Omega} u^{\gamma+1}(t)+C\left(\epsilon, \gamma,\left\|u_{0}\right\|_{L^{\gamma}},\left\|u_{0}\right\|_{L^{1}}, A_{0}, A_{2}, a_{1},|\Omega|\right) .
$$

By choosing $\epsilon=\frac{4(\gamma-1)}{\gamma^{2}(1+\tilde{\mu})}$, we get

$$
\frac{1}{\gamma} \frac{d}{d t} \int_{\Omega} u^{\gamma}(t) \leq-\int_{\Omega} u^{\gamma+1}(t)+C\left(\gamma,\left\|u_{0}\right\|_{L^{\gamma}}\right) \leq-\frac{1}{|\Omega|^{\frac{1}{\gamma}}}\left(\int_{\Omega} u^{\gamma}(t)\right)^{\frac{\gamma+1}{\gamma}}+C\left(\gamma,\left\|u_{0}\right\|_{L^{\gamma}}\right) .
$$

This implies that

$$
\int_{\Omega} u^{\gamma}(t) \leq C\left(\gamma,\left\|u_{0}\right\|_{L^{\gamma}},\left\|u_{0}\right\|_{L^{1}}, A_{0}, A_{2}, a_{1},|\Omega|\right) \quad \forall t \in\left[t_{0}, t_{0}+T_{\max }\right) .
$$

(4.22) then follows.

Step 3. In this sept, we prove that there is $C=C\left(\left\|u_{0}\right\|_{L^{\infty}}\right)$ such that

$$
\|u(t)\|_{C^{0}(\bar{\Omega})}+\|v(t)\|_{C^{0}(\bar{\Omega})} \leq C \quad \forall t \in\left[t_{0}, t_{0}+T_{\max }\right) .
$$

By the variation of constant formula, we have 


$$
\begin{aligned}
u(t) & =e^{-A\left(t-t_{0}\right)} u_{0}-\chi \int_{t_{0}}^{t} e^{-(t-s) A} \nabla(u(s) \cdot \nabla v(s)) d s \\
& +\int_{t_{0}}^{t} e^{-A(t-s)} u(s)[\underbrace{1+a_{0}(s, \cdot)-a_{1}(s, \cdot) u(s)-\left(a_{2}(s, \cdot)\right)_{+} \int_{\Omega} u(s)+\left(a_{2}(s, \cdot)\right)_{-} \int_{\Omega} u(s)}_{I_{0}(\cdot, s)}] d s,
\end{aligned}
$$

Note that $u(s) I_{0}(\cdot, s) \leq u(s)[\underbrace{1+A_{2} M_{0}+a_{0}(\cdot, s)-a_{1}(s, \cdot) u(s)}_{I_{1}(\cdot, s)}]$ and by parabolic comparison principle, we get $\int_{t_{0}}^{t} e^{-A(t-s)} u(s) I_{0}(\cdot, s) d s \leq \int_{t_{0}}^{t} e^{-A(t-s)} u(s) I_{1}(\cdot, s) d s$. Therefore

$$
u(t) \leq u_{1}(t)+u_{2}(t)+u_{3}(t)
$$

where

$$
u_{1}(t)=e^{-A\left(t-t_{0}\right)} u_{0}, \quad u_{2}(t)=-\chi \int_{t_{0}}^{t} e^{-(t-s) A} \nabla(u(s) \cdot \nabla v(s)) d s
$$

and

$$
u_{3}(t, x)=\int_{t_{0}}^{t} e^{-A(t-s)} u(s)\left[1+A_{2} M_{0}+a_{0}(\cdot, s)-a_{1}(s, \cdot) u(s)\right] d s .
$$

Note that there are $c_{0}, c_{1}>0$ such that $\left(1+A_{2} M_{0}+a_{0}(t, x)\right) r-a_{1}(t, x) r^{2} \leq c_{0}-c_{1} r^{2}$ for all $t \in \mathbb{R}, x \in \Omega$, and $r \geq 0$. We then have that

$$
\left\|u_{1}(t)\right\|_{L^{\infty}(\Omega)} \leq\left\|u_{0}\right\|_{L^{\infty}(\Omega)} \quad \forall t \in\left[t_{0}, t_{0}+T_{\max }\right)
$$

and

$$
u_{3}(t) \leq C \int_{t_{0}}^{t} e^{-A(t-s)} d s \leq C \int_{t_{0}}^{t} e^{-(t-s)} \leq C \quad \forall t \in\left[t_{0}, t_{0}+T_{\max }\right) .
$$

Choose $p>n$ and $\alpha \in\left(\frac{n}{2 p}, \frac{1}{2}\right)$. Then $X^{\alpha} \subset L^{\infty}(\Omega)$ and the inclusion is continuous (see [11] exercise 10, page 40.) Choose $\epsilon \in\left(0, \frac{1}{2}-\alpha\right)$, then we have

$$
\begin{aligned}
\left\|u_{2}(t)\right\|_{L^{\infty}(\Omega)} & \leq C\left\|A^{\alpha} u_{2}(t)\right\|_{L^{p}(\Omega)} \leq C \chi \int_{t_{0}}^{t}\left\|A^{\alpha} e^{-(t-s) A} \nabla(u(s) \cdot \nabla v(s))\right\|_{L^{p}(\Omega)} d s \\
& \leq C \int_{t_{0}}^{t}(t-s)^{-\alpha-\frac{1}{2}-\epsilon} e^{-\mu(t-s)}\|u(s) \cdot \nabla v(s)\| \|_{L^{p}(\Omega)} d s \\
& \leq C \int_{t_{0}}^{t}(t-s)^{-\alpha-\frac{1}{2}-\epsilon} e^{-\mu(t-s)}\|u(s)\|_{L^{p_{1}}(\Omega)}\|\nabla v(s)\|_{L^{p_{2}(\Omega)}} d s
\end{aligned}
$$

for $t \in\left[t_{0}, t_{0}+T_{\max }\right)$, where $p_{1}>p$ and $\frac{1}{p}=\frac{1}{p_{1}}+\frac{1}{p_{2}}$. Note that $\|\nabla v(s)\|_{L^{p_{2}(\Omega)}} \leq C\|u(s)\|_{L^{p_{2}}(\Omega)}$. By (4.22), we get

$$
\left\|u_{2}(t)\right\|_{L^{\infty}(\Omega)} \leq C\left(\left\|u_{0}\right\|_{L^{\infty}(\Omega)}\right) \int_{t_{0}}^{\infty}(t-s)^{-\alpha-\frac{1}{2}-\epsilon} e^{-\mu(t-s)} d s<\infty .
$$

Therefore

$$
\|v(t)\|_{\infty} \leq\|u(t)\|_{L^{\infty}(\Omega)} \leq C\left(\left\|u_{0}\right\|_{L^{\infty}(\Omega)}\right) \quad \forall t \in\left[t_{0}, t_{0}+T_{\max }\right) .
$$

(4.23) then follows. Theorem 1.2(2) is thus proved. 
Remark 4.1. (1) For the proof of Theorem 1.2(1) in the case $\chi \leq 0$, we need to write

$$
-\chi u v+(\chi)_{+} \bar{u} \underline{u}-(\chi)_{-} u^{2}=-(\chi)_{+} u(v-\underline{u})-(\chi)_{+} \underline{u} \bar{U}-(\chi)_{-} u \bar{U}+(\chi)_{-} u(v-\bar{u})
$$

and the proof follows as in the case $\chi>0$.

(2) For the proof of Theorem 1.2(2) in the case $\chi \leq 0$, the only major change is in Step 1. Indeed in Step 1, we need to consider $\gamma \in(1, \infty)$ and use [52, Lemma 3.2]. The proof then follows as in the case $\chi>0$.

(3) Assume (H2)'. It follows from the proof of Theorem 1.2. (2) that for any

$M \geq \frac{a_{0, \text { sup }}}{\inf _{t \in \mathbb{R}}\left\{a_{1, \text { inf }}(t)-|\Omega|\left(a_{2, \text { inf }}(t)\right)_{-}\right\}}$, there is a positive constant $C=C(M)$ depending only on $M$ such that for any $u_{0} \in C^{0}(\bar{\Omega})$ with $u_{0} \geq 0$ and $\left\|u_{0}\right\|_{C^{0}(\bar{\Omega})} \leq M, 0 \leq u\left(\cdot, t ; t_{0} ; u_{0}\right) \leq C$.

\section{Existence of entire positive solutions}

In this section, we explore the existence of entire positive solutions of (1.3) in the general case; the existence of time almost periodic, time periodic, and time independent positive solutions of (1.3) in the case that the coefficients of (1.3) are time almost periodic, time periodic, and time independent, respectively; and prove Theorem 1.3.

We first prove three lemmas. Throughout this section, we assume that $\chi>0$ (the case $\chi \leq 0$ follows from similar arguments), (H2) holds and we let

$$
M=\frac{a_{0, \text { sup }}}{\inf _{t \in \mathbb{R}}\left\{a_{1, \text { inf }}(t)-|\Omega|\left(a_{2, \text { inf }}(t)\right)_{-}\right\}-\chi} .
$$

Let $\left(u\left(x, t ; t_{0}, u_{0}\right), v\left(x, t ; t_{0}, u_{0}\right)\right)$ be the solution of (1.3) with $u\left(x, t_{0} ; t_{0}, u_{0}\right)=u_{0}(x)\left(u_{0} \in\right.$ $\left.C^{0}(\bar{\Omega})\right)$. By Corollary 3.1, for any $t_{2}>t_{1}>t_{0}$,

$$
u\left(x, t_{2} ; t_{0}, u_{0}\right)=u\left(x, t_{2} ; t_{1}, u\left(\cdot, t_{1} ; t_{0}, u_{0}\right)\right) .
$$

By Theorem 1.2, the global existence of (1.3) holds, and for any $0 \leq u_{0}(\cdot) \leq M$,

$$
0 \leq u\left(\cdot, t ; t_{0}, u_{0}\right) \leq M \quad \text { for } \quad t \geq t_{0} .
$$

Lemma 5.1. Fix a $T>0$. For any $\epsilon>0$, there is $\delta=\delta(T)>0$ such that for any give $u_{0}(\cdot) \geq 0$ with $\sup u_{0}<\delta$ and any $t_{0} \in \mathbb{R}, u\left(x, t+t_{0} ; t_{0}, u_{0}\right)<\epsilon$ for $0 \leq t \leq T$.

Proof. It follows from the continuity with respect to initial conditions.

Fix a $T>0$. Fix $\epsilon_{0}$ such that $\epsilon_{0}<\frac{a_{0, \text { inf }}}{\chi+|\Omega| \cdot\left|a_{2, \text { sup }}\right|}$. Let $\delta_{0}=\delta$ be as in Lemma 5.1 with $\epsilon=\epsilon_{0}$. By Lemma 5.1, for given $0 \leq u_{0}(x)<\delta_{0}, u\left(x, t+t_{0} ; t_{0}, u_{0}\right)<\epsilon_{0}$ for $0 \leq t \leq T$. This implies that $v\left(x, t+t_{0} ; t_{0}, u_{0}\right)=V\left(u\left(\cdot, t+t_{0} ; t_{0}, u_{0}\right)\right):=A^{-1} u\left(\cdot, t+t_{0} ; t_{0}, u_{0}\right)<\epsilon_{0}$ for $0 \leq t \leq T$.

Lemma 5.2. For any $t_{0} \in \mathbb{R}$ and any $0<u_{0}(x)<\min \left\{\delta_{0}, \frac{a_{0, \inf }-\epsilon_{0}\left(\chi+|\Omega| \cdot\left|a_{2, \text { sup }}\right|\right)}{a_{1, \text { sup }}}\right\}$ for $x \in \Omega$, $u\left(x, t+t_{0} ; t_{0}, u_{0}\right)>\inf u_{0}$ for $0<t \leq T$ and $x \in \Omega$. 
Proof. By Lemma 5.1, $V\left(u\left(\cdot, t+t_{0} ; t_{0}, u_{0}\right)\right)<\epsilon_{0}$ for $0 \leq t \leq T$. Hence

$$
\begin{aligned}
u_{t} & =\Delta u-\chi \nabla u \cdot \nabla V(u)-\chi u(V(u)-u)+u\left(a_{0}(t, x)-a_{1}(t, x) u-a_{2}(t, x) \int_{\Omega} u\right) \\
& \geq \Delta u-\chi \nabla u \cdot \nabla V(u)+u\left(a_{0}(t, x)-\epsilon_{0} \chi-a_{1}(t, x) u-a_{2}(t, x) \int_{\Omega} u\right) \\
& \geq \Delta u-\chi \nabla u \cdot \nabla V(u)+u\left(a_{0, \text { inf }}-\epsilon_{0}\left(\chi+|\Omega| \cdot\left|a_{2, \sup }\right|\right)-a_{1, \sup } u\right) .
\end{aligned}
$$

Then by comparison principle, we have

$$
u\left(x, t+t_{0} ; t_{0}, u_{0}\right) \geq u\left(t ; \inf u_{0}\right) \quad 0 \leq t \leq T
$$

where $u\left(t ; \inf u_{0}\right)$ is the solution of the ODE

$$
\dot{u}=u\left(a_{0, \text { inf }}-\epsilon_{0}\left(\chi+|\Omega| \cdot\left|a_{2, \text { sup }}\right|\right)-a_{1, \text { sup }} u\right) .
$$

with $u\left(0 ; \inf u_{0}\right)=\inf u_{0}$. Note that $u\left(t ; \inf u_{0}\right)$ increases as $t$ increases. The lemma then follows.

Lemma 5.3. There is $\delta^{*}=\delta^{*}(T)>0$ such that for any $0<\delta \leq \delta^{*}, t_{0} \in \mathbb{R}$, and $u_{0}(\cdot)$ with $\delta \leq \inf u_{0} \leq \sup u_{0} \leq M, u\left(x, t_{0}+T ; t_{0}, u_{0}\right) \geq \delta$ for $x \in \Omega$.

Proof. We prove the lemma by contradiction. Assume that the lemma does not hold. Then there are $\delta_{n} \rightarrow 0, t_{n} \in \mathbb{R}$, and $u_{n}(\cdot)$ with $\delta_{n} \leq \inf u_{n} \leq M$ such that inf $u\left(\cdot, t_{n}+T ; t_{n}, u_{n}\right)<\delta_{n}$. Without loss of generality, we assume that $\delta_{n}<\min \left\{\delta_{0}, \frac{a_{0, \text { inf }}-\epsilon_{0}\left(\chi+|\Omega| \cdot\left|a_{2, \text { sup }}\right|\right)}{a_{1, \text { sup }}}\right\}$. By Lemma [5.2, we must have $\sup u_{n} \geq \min \left\{\delta_{0}, \frac{a_{0, \text { inf }}-\epsilon_{0}\left(\chi+|\Omega| \cdot\left|a_{2, \text { sup }}\right|\right)}{a_{1, \text { sup }}}\right\}$. Let

$$
\Omega_{n}=\left\{x \in \Omega \mid u_{n}(x) \geq \frac{1}{2} \min \left\{\delta_{0}, \frac{a_{0, \text { inf }}-\epsilon_{0}\left(\chi+|\Omega| \cdot\left|a_{2, \text { sup }}\right|\right)}{a_{1, \text { sup }}}\right\}\right\} .
$$

Without loss of generality, we may assume that $m_{0}=\lim _{n \rightarrow \infty}\left|\Omega_{n}\right|$ exists, where $\left|\Omega_{n}\right|$ is the Lebesgue measure of $\Omega_{n}$. Assume that $m_{0}=0$. Then there is $\tilde{u}_{n} \in C^{0}(\bar{\Omega})$ such that

$$
\delta_{n} \leq \tilde{u}_{n}(x) \leq \frac{1}{2} \min \left\{\delta_{0}, \frac{a_{0, \text { inf }}-\epsilon_{0}\left(\chi+|\Omega| \cdot\left|a_{2, \text { sup }}\right|\right)}{a_{1, \text { sup }}}\right\}
$$

and

$$
\lim _{n \rightarrow \infty}\left\|u_{n}-\tilde{u}_{n}\right\|_{L^{p}(\Omega)}=0 \quad \forall 1 \leq p<\infty
$$

This implies that

$$
\lim _{n \rightarrow \infty}\left\|u\left(\cdot, t ; t_{n}, u_{n}\right)-u\left(\cdot, t ; t_{n}, \tilde{u}_{n}\right)\right\|_{L^{p}(\Omega)}=0
$$

uniformly in $t \in\left[t_{n}, t_{n}+T\right]$ for all $1 \leq p<\infty$. Indeed, let $G(\cdot)$ be as in the proof of Theorem 1.1(1). Then $G\left(u\left(\cdot, t ; t_{n}, u_{n}\right)\right)(t)=u\left(\cdot, t ; t_{n}, u_{n}\right), G\left(u\left(\cdot, t ; t_{n}, \tilde{u}_{n}\right)\right)(t)=u\left(\cdot, t ; t_{n}, \tilde{u}_{n}\right)$. Let

$$
\begin{gathered}
\hat{G}\left(u_{n}\right)(t)=G\left(u\left(\cdot, t ; t_{n}, u_{n}\right)\right)(t), \quad \hat{G}\left(\tilde{u}_{n}\right)(t)=G\left(u\left(\cdot, t ; t_{n}, \tilde{u}_{n}\right)\right)(t), \\
w_{n}(\cdot, t)=G\left(u\left(\cdot, t ; t_{n}, u_{n}\right)\right)(t)-G\left(u\left(\cdot, t ; t_{n}, \tilde{u}_{n}\right)\right)(t)
\end{gathered}
$$


and

$$
W_{n}(\cdot, t)=V\left(G\left(u\left(\cdot, t ; t_{n}, u_{n}\right)\right)(t)\right)-V\left(G\left(u\left(\cdot, t ; t_{n}, \tilde{u}_{n}\right)\right)(t)\right)
$$

Then

$$
\begin{aligned}
& w_{n}(\cdot, t)= \\
& e^{-A\left(t-t_{n}\right)}\left(u_{n}-\tilde{u}_{n}\right)-\chi \int_{t_{n}}^{t} e^{-A(t-s)} \nabla\left[w_{n}(\cdot, s) \cdot \nabla V\left(\hat{G}\left(u_{n}\right)(s)+\hat{G}\left(\tilde{u}_{n}\right)(s) \cdot \nabla W_{n}(\cdot, s)\right] d s\right. \\
& +\int_{t_{n}}^{t} e^{-A(t-s)} w_{n}(\cdot, s)\left(1+a_{0}(s, \cdot)-a_{1}(s, \cdot)\left(\hat{G}\left(u_{n}\right)+\hat{G}\left(\tilde{u}_{n}\right)\right)(s)-a_{2}(s, \cdot) \int_{\Omega} \hat{G}\left(u_{n}\right)(s)\right) d s \\
& -\int_{t_{n}}^{t} e^{-A(t-s)} a_{2}(s, \cdot)\left(\int_{\Omega} w_{n}(\cdot, s)\right) \hat{G}\left(\tilde{u}_{n}\right)(s) d s .
\end{aligned}
$$

Now, fix $1<p<\infty$. By regularity and a priori estimates for elliptic equations, [11, Theorem 1.4.3], Lemma 2.2, and (5.4), for any $\epsilon \in\left(0, \frac{1}{2}\right)$, we have

$$
\begin{aligned}
& \left\|w_{n}(\cdot, t)\right\|_{L^{p}(\Omega)} \\
& \leq\left\|u_{n}-\tilde{u}_{n}\right\|_{L^{p}(\Omega)}+C \chi_{t_{n} \leq s \leq t_{n}+T}\left\|\nabla V\left(\hat{G}\left(u_{n}\right)(s)\right)\right\|_{C^{0}(\bar{\Omega})} \int_{t_{n}}^{t}(t-s)^{-\epsilon-\frac{1}{2}}\left\|w_{n}(\cdot, s)\right\|_{L^{p}(\Omega)} d s \\
& +C \chi_{t_{n} \leq s \leq t_{n}+T}\left\|\hat{G}\left(\tilde{u}_{n}\right)(s)\right\|_{C^{0}(\bar{\Omega})} \int_{t_{n}}^{t}(t-s)^{-\epsilon-\frac{1}{2}}\left\|w_{n}(\cdot, s)\right\|_{L^{p}(\Omega)} d s \\
& +C \int_{t_{n}}^{t}\left\{1+A_{0}+A_{1}\left[\max _{t_{n} \leq s \leq t_{n}+T}\left(\left\|\hat{G}\left(u_{n}\right)(s)\right\|_{C^{0}(\bar{\Omega})}+\left\|\hat{G}\left(\tilde{u}_{n}\right)(s)\right\|_{C^{0}(\bar{\Omega})}\right)\right]\right\}\left\|w_{n}(\cdot, s)\right\|_{L^{p}(\Omega)} d s \\
& +C \int_{t_{n}}^{t} A_{2}|\Omega| \max _{t_{n} \leq s \leq t_{n}+T}\left\|\hat{G}\left(u_{n}\right)(s)\right\|_{C^{0}(\bar{\Omega})}\left\|w_{n}(\cdot, s)\right\|_{L^{p}(\Omega)} d s \\
& +C \int_{t_{n}}^{t} A_{2}\left\|\hat{G}\left(\tilde{u}_{n}\right)(s)\right\|_{C^{0}(\bar{\Omega})}\left\|w_{n}(\cdot, s)\right\|_{L^{p}(\Omega)} d s .
\end{aligned}
$$

Therefore there exists a positive constant $C_{0}$ independent of $t$ and $n$ such that

$$
\left\|w_{n}\left(\cdot, t+t_{n}\right)\right\|_{L^{p}(\Omega)} \leq\left\|u_{n}-\tilde{u}_{n}\right\|_{L^{p}(\Omega)}+C_{0} \int_{0}^{t}(t-s)^{-\epsilon-\frac{1}{2}}\left\|w_{n}\left(\cdot, s+t_{n}\right)\right\|_{L^{p}(\Omega)} d s \quad \forall t \in[0, T] .
$$

By (5.6) and the generalized Gronwall's inequality (see [11, page 6]), we get

$$
\lim _{n \rightarrow \infty}\left\|u\left(\cdot, t ; t_{n}, u_{n}\right)-u\left(\cdot, t ; t_{n}, \tilde{u}_{n}\right)\right\|_{L^{p}(\Omega)}=0,
$$

uniformly in $t \in\left[t_{n}, t_{n}+T\right]$ for all $1 \leq p<\infty$. Therefore,

$$
\lim _{n \rightarrow \infty}\left\|V\left(u\left(\cdot, t ; t_{n}, u_{n}\right)\right)-V\left(u\left(\cdot, t ; t_{n} ; \tilde{u}_{n}\right)\right)\right\|_{C^{1}(\bar{\Omega})}=0
$$

uniformly in $t \in\left[t_{n}, t_{n}+T\right]$. Note that

$$
V\left(u\left(\cdot, t ; t_{n}, \tilde{u}_{n}\right)\right)(x) \leq \epsilon_{0}
$$

for all $t \in\left[t_{n}, t_{n}+T\right]$ and $x \in \Omega$. It then follows that

$$
V\left(u\left(\cdot, t ; t_{n}, u_{n}\right)\right)(x) \leq 2 \epsilon_{0}
$$


for all $t \in\left[t_{n}, t_{n}+T\right], x \in \Omega$, and $n \gg 1$. Then by the arguments of Lemma 5.2, inf $u\left(\cdot, t_{n}+\right.$ $\left.T ; t_{n}, u_{n}\right) \geq \delta_{n}$, which is a contradiction. Therefore, $m_{0} \neq 0$.

By $m_{0} \neq 0$ and comparison principle for parabolic equations, without loss of generality, we may assume that

$$
\liminf _{n \rightarrow \infty}\left\|e^{-A t} u_{n}\right\|_{C^{0}(\bar{\Omega})}>0 \quad \forall t \in[0, T] .
$$

This together with the arguments in the proof of Theorem 1.1(2) implies that there is $T_{0}>0$ and $\delta_{\infty}>0$ such that

$$
\sup u\left(\cdot, t_{n}+T_{0} ; t_{n}, u_{n}\right) \geq \delta_{\infty}
$$

for all $n \gg 1$. By a priori estimates for parabolic equations, without loss of generality, we may assume that

$$
u\left(\cdot, t_{n}+T_{0} ; t_{n}, u_{n}\right) \rightarrow u_{0}^{*}, \quad u\left(\cdot, t_{n}+T ; t_{n}, u_{n}\right) \rightarrow u^{*}
$$

as $n \rightarrow \infty$. By (H1), without loss of generality, we may also assume that

$$
a_{i}\left(t+t_{n}, \cdot\right) \rightarrow a_{i}^{*}(t, x)
$$

as $n \rightarrow \infty$ locally uniformly in $(t, x) \in \mathbb{R} \times \bar{\Omega}$. Then by Corollary 3.1 ,

$$
u^{*}(x)=u^{*}\left(x, T ; T_{0}, u_{0}^{*}\right) \text { and } \inf u^{*}=0,
$$

where $\left(u^{*}\left(x, t ; T_{0}, u_{0}^{*}\right), v^{*}\left(x, t ; T_{0}, u_{0}^{*}\right)\right)$ with $v^{*}\left(\cdot, t ; T_{0}, u_{0}^{*}\right)=A^{-1} u^{*}\left(\cdot, t ; T_{0}, u_{0}^{*}\right)$ is the solution of (1.3) with $a_{i}(t, x)$ being replaced by $a^{*}(t, x)$. By comparison principle, we must have $u_{0}^{*} \equiv 0$. But

$$
\sup u_{0}^{*} \geq \delta_{\infty}
$$

This is a contradiction.

Proof of Theorem 1.3. We first prove the existence of entire positive solutions of (1.3) in the general case.

Let $\delta^{*}>0$ be given by Lemma 5.3 with $T=1$. Choose $u_{0} \in C^{0}(\bar{\Omega})$ such that $\delta^{*} \leq u_{0}(x) \leq M$. By Lemma 5.3 and (5.2),

$$
\delta^{*} \leq u\left(x, t_{0}+n ; t_{0}, u_{0}\right) \leq M \quad \forall x \in \bar{\Omega}, t_{0} \in \mathbb{R}, n \in \mathbb{N} .
$$

Set $t_{n}=-n$ and define $u_{n}(x)=u\left(x, 0 ; t_{n}, u_{0}\right)$. Choose $\tilde{t}$ such that $-2<\tilde{t}<-1$. Then there is $\tilde{M}>0$ such that for each $n \geq 3$, we have

$$
\left\|u_{n}\right\|_{\alpha}=\left\|u\left(\cdot, 0 ; t_{n}, u_{0}\right)\right\|_{\alpha}=\left\|u\left(\cdot, 0 ; \tilde{t}, u\left(\cdot, \tilde{t} ; t_{n}, u_{0}\right)\right)\right\|_{\alpha} \leq \tilde{M} .
$$

Therefore by Arzela-Ascoli Theorem, there exist $n_{k}, u_{0}^{*} \in C^{0}(\bar{\Omega})$ such that $u_{n_{k}}$ converges to $u_{0}^{*}$ in $C^{0}(\bar{\Omega})$ as $n_{k} \rightarrow \infty$. Then by Corollary 3.1 , we have

$$
u\left(\cdot, t ; t_{n_{k}}, u_{0}\right)=u\left(\cdot, t ; 0, u\left(\cdot, 0 ; t_{n_{k}}, u_{0}\right)\right)=u\left(\cdot, t ; 0, u_{n_{k}}\right) \rightarrow u\left(\cdot, t ; 0, u_{0}^{*}\right)
$$

in $C^{0}(\bar{\Omega})$ as $n \rightarrow \infty$ for $t \geq 0$. Moreover, by (5.2) and Lemma 5.3 ,

$$
\delta^{*} \leq u\left(x, n ; 0, u_{0}^{*}\right) \leq M \quad \forall x \in \bar{\Omega}, n \in \mathbb{N} .
$$


We need to prove that $u\left(\cdot, t ; 0, u_{0}^{*}\right)$ has backward extension. To see that, fix $m \in \mathbb{N}$. Then $u\left(\cdot, t ; t_{n}, u_{0}\right)$ is defined for $t>-m$ and $n>m$. Observe that

$$
u_{n}(\cdot)=u\left(\cdot, 0 ; t_{n}, u_{0}\right)=u\left(\cdot, 0 ;-m, u\left(\cdot,-m ; t_{n}, u_{0}\right)\right) .
$$

Without loss of generality, we may assume that $u\left(\cdot,-m ; t_{n_{k}}, u_{0}\right) \rightarrow u_{m}^{*}(\cdot)$ in $C^{0}(\bar{\Omega})$. Then

$$
u\left(\cdot, t ; t_{n_{k}}, u_{0}\right)=u\left(\cdot, t ;-m, u\left(\cdot,-m ; t_{n_{k}}, u_{0}\right)\right) \rightarrow u\left(\cdot, t ;-m, u_{m}^{*}\right)
$$

for $t>-m$ and $u\left(\cdot, t ; 0, u_{0}^{*}\right)=u\left(\cdot, t ;-m, u_{m}^{*}\right)$ for $t \geq 0$. This implies that $u^{*}\left(x, t ; 0, u_{0}^{*}\right)$ has a backward extension up to $t=-m$. Let $m \rightarrow \infty$, we have that $u^{*}(x, t)$ has a backward extension on $(-\infty, 0)$.

Let $u^{*}(x, t)=u^{*}\left(x, t ; 0, u_{0}^{*}\right)$ and $v^{*}(x, t)=A^{-1} u^{*}(\cdot, t)$, Then $\left(v^{*}(x, t), u^{*}(x, t)\right)$ is an entire nonnegative solution of (1.3). Moreover,

$$
\delta^{*} \leq u^{*}(x, n) \leq M \quad \forall x \in \bar{\Omega}, n \in \mathbb{Z} .
$$

This implies that

$$
0<\inf _{x \in \bar{\Omega}, t \in \mathbb{R}} u^{*}(x, t) \leq M, \quad 0<\inf _{x \in \bar{\Omega}, t \in \mathbb{R}} v^{*}(x, t) \leq M .
$$

Therefore, $\left(v^{*}(x, t), u^{*}(x, t)\right)$ is an entire positive bounded solution of (1.3).

Next, we prove $(1),(2)$, and (3).

(1) Assume that $a_{i}(t+T, x)=a_{i}(t, x)$ for $i=0,1,2$. Let $\delta^{*}=\delta^{*}(T)>0$ be given by Lemma 5.3 and set

$$
E(T)=\left\{u_{0} \in C^{0}(\bar{\Omega}): \delta^{*} \leq u_{0} \leq M\right\} .
$$

Note that $E(T)$ is nonempty, closed, convex and bounded subset of $C^{0}(\bar{\Omega})$. Define the map $\mathcal{T}(T): E(T) \rightarrow C^{0}(\bar{\Omega})$ by $\mathcal{T}(T) u_{0}=u\left(\cdot, T ; 0, u_{0}\right)$. Note that $\mathcal{T}(T)$ is well defined and continuous by continuity with respect to initial conditions.

Let $u_{0} \in E(T)$. Then by Theorem 1.2 , we have $0<u\left(\cdot, T ; 0, u_{0}\right) \leq M$ and by Lemma 5.3, we have $u\left(\cdot, T ; 0, u_{0}\right) \geq \delta^{*}$. Thus $u\left(\cdot, T ; 0, u_{0}\right) \in E(T)$ and $\mathcal{T}(T) E(T) \subset E(T)$.

Let $\frac{n}{2 p}<\alpha<\frac{1}{2}$, and $\epsilon \in\left(0, \frac{1}{2}-\alpha\right)$. By the similar arguments as those in the proof of local existence, we have that

$$
\left\|u\left(\cdot, T ; 0, u_{0}\right)\right\|_{\alpha} \leq C M T^{-\alpha}+C M^{2} T^{\frac{1}{2}-\alpha-\epsilon}+C M\left[1+A_{0}+k_{1}\left(A_{1}+|\Omega| A_{2}\right)\right] T^{1-\alpha} .
$$

Now choose $\nu$ such that $0 \leq \nu<2 \alpha-\frac{n}{p}$, then $X^{\alpha} \subset C^{\nu}(\bar{\Omega})$, where the inclusion is continuous. Thus by Arzela-Ascoli Theorem, $\mathcal{T}(T) E(T)$ is precompact. Therefore by Schauder fixed point theorem, there exists $u^{T} \in E(T)$ such that $\mathcal{T}(T) u^{T}=u^{T}$, i.e $u\left(\cdot, T ; 0, u^{T}\right)=u^{T}(\cdot)$. Since $u\left(\cdot, t+T ; 0, u^{T}\right)=u\left(\cdot, t ; T, u\left(\cdot, T ; 0, u^{T}\right)\right)=u\left(\cdot, t ; 0, u^{T}\right), u\left(\cdot, t ; 0, u^{T}\right)$ is periodic with period $T$. Now from the facts that $u\left(., t ; 0, u^{T}\right)$ is periodic with period $T$ and the uniqueness of solutions of

$$
\left\{\begin{array}{l}
-\Delta v+v=u\left(x, t ; 0, u^{T}\right) \quad x \in \Omega \\
\frac{\partial v}{\partial n}=0 \text { on } \partial \Omega,
\end{array}\right.
$$

we get $v\left(\cdot, t ; 0, u^{T}\right)=A^{-1} u\left(\cdot, t ; 0, u^{T}\right)$ is periodic with period $T$. Then $\left(u\left(\cdot, t ; 0, u^{T}\right), v\left(\cdot, t ; 0, u^{T}\right)\right)$ is a positive periodic solution of (1.3). 
(2) Assume that $a_{i}(t, x) \equiv a_{i}(t)$. Note that in this case, every solution of the ODE

$$
u_{t}=u\left(a_{0}(t)-\left(a_{1}(t) u+|\Omega| a_{2}(t)\right) u\right)
$$

is a solution of the first equation of the system (1.3) with Neumann boundary. (2) then follows from Lemma 2.5 .

(3) Assume that $a_{i}(t, x) \equiv a_{i}(x)(i=0,1,2)$. In this case, each $\tau>0$ is a period for $a_{i}$. By (2), there exist $u^{\tau} \in E(\tau)$ such that $\left(u\left(\cdot, t ; 0, u^{\tau}\right), A^{-1}\left(u\left(\cdot, t ; 0, u^{\tau}\right)\right)\right)$ is a positive periodic solution of (1.3) with period $\tau$. Note that there is $\tilde{M}>0$ such that for each $\tau>0$ and $u_{0} \in E(\tau)$, $\left\|u\left(\cdot, t ; 0, u_{0}\right)\right\|_{\alpha} \leq \tilde{M}$ for each $1 \leq t \leq 2$. Let $\tau_{n}=\frac{1}{n}$, then there exists $u_{n} \in E\left(\tau_{n}\right)$ such that $u\left(\cdot, t ; 0, u_{n}\right)$ is periodic with period $\tau_{n}$ and

$$
\left\|u_{n}\right\|_{\alpha}=\left\|u\left(\cdot, \tau_{n} ; 0, u_{n}\right)\right\|_{\alpha}=\left\|u\left(\cdot, N \tau_{n} ; 0, u_{n}\right)\right\|_{\alpha} \leq \tilde{M},
$$

where $N$ is such that $1 \leq N \tau_{n} \leq 2$.

We claim that there is $\delta>0$ such that

$$
\left\|u_{n}(\cdot)\right\|_{C^{0}(\bar{\Omega})} \geq \delta \quad \forall n \geq 1 .
$$

Suppose by contradiction that this does not hold. Then there exists $n_{k}$ such that $\left\|u_{n_{k}}\right\|_{C^{0}(\bar{\Omega})}<$ $\frac{1}{n_{k}}$ for every $k \geq 1$. Let $k_{0}$ such that $\frac{1}{n_{k}}<\epsilon_{0}$ for all $k \geq k_{0}$. By the proof of Lemma 5.2 we get that $u\left(\cdot, t ; 0, u_{n_{k}}\right) \geq u\left(t ; \inf u_{n_{k}}\right)$ for all $t>0$ and $k \geq k_{0}$, where $u\left(t ; \inf u_{n_{k}}\right)$ is the solution of (5.3) with $u\left(0 ; \inf u_{n_{k}}\right)=\inf u_{n_{k}}$. Let $\delta_{*}=\frac{a_{0, \text { inf }}-\epsilon_{0}\left(\chi+|\Omega| \cdot\left|a_{2, \text { sup }}\right|\right)}{2 a_{1, \text { sup }}}$ and choose $k$ large enough such that $\frac{1}{n_{k}}<\delta_{*}$. There is $t_{0}>0$ such that $u\left(t ; \inf u_{n_{k}}\right)>\delta_{*}$ for all $t \geq t_{0}$. Then we have

$$
u_{n_{k}}(x)=u\left(\cdot, m \tau_{n_{k}} ; 0, u_{n_{k}}\right) \geq u\left(m \tau_{n_{k}} ; \inf u_{n_{k}}\right)>\delta^{*}
$$

for all $m \in \mathbb{N}$ satisfying that $m \tau_{n_{k}}>t_{0}$. This is a contradiction. Therefore, (5.12) holds.

By (5.11) and Arzela-Ascoli theorem, there exist $n_{k}, u^{*} \in C^{0}(\bar{\Omega})$ such that $u_{n_{k}}$ converges to $u^{*}$ in $C^{0}(\bar{\Omega})$. By (15.12), $\left\|u^{*}(\cdot)\right\|_{C^{0}(\bar{\Omega})} \geq \frac{\delta}{2}$. We claim that $\left(u\left(\cdot, t ; 0, u^{*}\right), v\left(\cdot, t ; 0, u^{*}\right)\right)$ with $v\left(\cdot, t ; 0, u^{*}\right)=A^{-1} u\left(\cdot, t ; 0, u^{*}\right)$ is a steady state solution of (1.3) , that is,

$$
u\left(\cdot, t ; 0, u^{*}\right)=u^{*}(\cdot) \quad \text { for all } t \geq 0 .
$$

In fact, let $\epsilon>0$ be fix and let $t>0$. Note that

$$
\left[n_{k} t\right] \tau_{n_{k}}=\frac{\left[n_{k} t\right]}{n_{k}} \leq t \leq \frac{\left[n_{k} t\right]+1}{n_{k}}=\left(\left[n_{k} t\right]+1\right) \tau_{n_{k}} .
$$

By Corollary 3.1, we can choose $k$ large enough such that $\left|u\left(x, t ; 0, u^{*}\right)-u\left(x, t ; 0, u_{n_{k}}\right)\right|<\epsilon, \quad\left|u_{n_{k}}(x)-u^{*}(x)\right|<\epsilon, \quad\left|u\left(x, \frac{\left[n_{k} t\right]}{n_{k}} ; 0, u_{n_{k}}\right)-u\left(x, t ; 0, u_{n_{k}}\right)\right|<\epsilon$ for all $x \in \bar{\Omega}$. We then have

$$
\begin{aligned}
\left|u\left(x, t ; 0, u^{*}\right)-u^{*}\right| \leq & \left|u\left(x, t ; 0, u^{*}\right)-u\left(x, t ; 0, u_{n_{k}}\right)\right|+\left|u\left(x, t ; 0, u_{n_{k}}\right)-u\left(x,\left[n_{k} t\right] \tau_{n_{k}} ; 0, u_{n_{k}}\right)\right| \\
& +\left|u_{n_{k}}(x)-u^{*}(x)\right|<3 \epsilon \quad \forall x \in \bar{\Omega} .
\end{aligned}
$$

Letting $\epsilon \rightarrow 0$, (5.13) follows.

Remark 5.1. It follows from the proof of the existence of entire positive solutions in Theorem 1.3 and Remark 4.1 that the existence of positive entire solutions also holds under the weaker condition $(\mathbf{H 2})^{\prime}$. 


\section{$6 \quad$ Asymptotic Stability of Solutions}

In this section, we investigate the stability and uniqueness of entire positive solutions of (1.3), the asymptotic behavior of global positive solutions of (1.3), and prove Theorems 1.4 and 1.5 , Without loss of generality, we suppose throughout this section that $\chi>0$, since the arguments for $\chi \leq 0$ are similar. We first prove Theorem 1.5 .

Proof of Theorem 1.5. Suppose that (1.16) holds. For given $u_{0} \in C^{0}(\bar{\Omega})$ with $u_{0}(x) \geq 0, u_{0}(\cdot) \neq$ 0 , and $t_{0} \in \mathbb{R}$, let $\left(u\left(\cdot, t ; t_{0}, u_{0}\right), v\left(\cdot, t ; t_{0}, u_{0}\right)\right)$ be the solution of (1.3) satisfying the properties in Theorem 1.1(2). By Theorem 1.2. $\left(u\left(\cdot, t ; t_{0}, u_{0}\right), v\left(\cdot, t ; t_{0}, u_{0}\right)\right)$ exists for all $t>t_{0}$. Note that $u\left(x, t ; t_{0}, u_{0}\right)>0$ for all $x \in \bar{\Omega}$ and $t>t_{0}$. Without loss of generality, we may assume that $u_{0}(x)>0$ for all $x \in \bar{\Omega}$.

Let $(\bar{u}(t), \underline{u}(t))$ be as in (4.2). By Lemma 4.1 and the proof of Theorem 1.2 .

$$
\underline{u}(t) \leq u\left(x, t ; t_{0}, u_{0}\right) \leq \bar{u}(t) \quad \forall x \in \bar{\Omega}, t \geq t_{0} .
$$

Let $r_{1}$ and $r_{2}$ be as in (1.17) and (1.25), respectively.

(1) By Lemma 2.6 (1) and (2), for any $\epsilon>0$, there is $t_{\epsilon}>0$ such that

$$
r_{2}-\epsilon \leq \underline{u}(t) \leq \bar{u}(t) \leq r_{1}+\epsilon \quad \text { for } \quad t \geq t_{0}+t_{\epsilon} .
$$

(1) then follows from (6.1) and (6.2).

(2) We first consider the case that $a_{i}(t, x)(i=0,1,2)$ are periodic in $t$ with period $T$. By Lemma 2.6(1), (2), and (3), there are periodic functions $m(t)$ and $M(t)$ with period $T$ such that

$$
r_{2} \leq m(t) \leq M(t) \leq r_{1} \quad \forall t \in \mathbb{R}
$$

and for any $\epsilon>0$, there is $t_{\epsilon}>0$ such that

$$
m(t)-\epsilon \leq \underline{u}(t) \leq \bar{u}(t) \leq M(t)+\epsilon \quad \forall t \geq t_{0}+t_{\epsilon} .
$$

In this case, (2) then follows from (6.1) and (6.3).

Next, we consider the cases that $a_{i}(t, x)(i=0,1,2)$ are almost periodic in $t$. By Lemma 2.6(1), (2), and (4), there are almost periodic functions $m(t)$ and $M(t)$ such that

$$
r_{2} \leq m(t) \leq M(t) \leq r_{1} \quad \forall t \in \mathbb{R}
$$

and for any $\epsilon>0$, there is $t_{\epsilon}>0$ such that (6.3) holds. (2) then follows from (6.1) and (6.3).

We now prove Theorem 1.4

Proof of Theorem 1.4. (1) Suppose that $a_{i}(t, x) \equiv a_{i}(t)$ for $i=0,1,2$ and

$$
\inf _{t \in \mathbb{R}}\left\{a_{1}(t)-|\Omega|\left|a_{2}(t)\right|\right\}>2 \chi .
$$

For given $u_{0} \in C^{0}(\bar{\Omega})$ with $u_{0}(x) \geq 0, u_{0}(\cdot) \neq 0$, and $t_{0} \in \mathbb{R}$, let $\left(u\left(\cdot, t ; t_{0}, u_{0}\right), v\left(\cdot, t ; t_{0}, u_{0}\right)\right)$ be the solution of (1.3) satisfying the properties in Theorem 1.1(2). Again, by Theorem 1.2, 
$\left(u\left(\cdot, t ; t_{0}, u_{0}\right), v\left(\cdot, t ; t_{0}, u_{0}\right)\right)$ exists for all $t>t_{0}$ and without loss of generality, we may assume that $u_{0}(x)>0$ for all $x \in \bar{\Omega}$.

Let $(\bar{u}(t), \underline{u}(t))$ be as in (4.2) . Let $\left(u^{*}(t), v^{*}(t)\right)$ be the unique entire positive spatially homogeneous solution of (1.3) in Theorem 1.3(2). By Lemma 4.1 and the proof of Theorem 1.2,

$$
\underline{u}(t) \leq u\left(x, t ; t_{0}, u_{0}\right) \leq \bar{u}(t) \quad \forall x \in \bar{\Omega}, t \geq t_{0} .
$$

By Lemma 2.6(1), for any $\epsilon>0$, there is $t_{\epsilon}>0$ such that

$$
\underline{u}(t)-\epsilon \leq u^{*}(t) \leq \bar{u}(t)+\epsilon \quad \forall t \geq t_{0}+t_{\epsilon} .
$$

By (6.5) and (6.6), it suffices to show $0 \leq \ln \frac{\bar{u}(t)}{\underline{\underline{u}(t)}} \longrightarrow 0$ as $t \rightarrow \infty$. Assume that $t>t_{0}$. By dividing the first equation of (4.1) by $\bar{u}$, and the second by $\underline{u}$, we get

$$
\left\{\begin{array}{l}
\frac{\bar{u}^{\prime}}{\bar{u}}=\left[a_{0}(t)-\left(a_{1}(t)-|\Omega|\left(a_{2}(t)\right)_{-}-\chi\right) \bar{u}-\left(|\Omega|\left(a_{2}(t)\right)_{+}+\chi\right) \underline{u}\right] \\
\underline{\underline{u}}=\left[a_{0}(t)-\left(a_{1}(t)-|\Omega|\left(a_{2}(t)\right)_{-}-\chi\right) \underline{u}-\left(|\Omega|\left(a_{2}(t)\right)_{+}+\chi\right) \bar{u}\right]
\end{array}\right.
$$

This together with (6.4) implies that

$$
\frac{d}{d t}\left(\ln \frac{\bar{u}}{\underline{u}}\right)=\frac{\bar{u}^{\prime}}{\bar{u}}-\frac{\underline{u}^{\prime}}{\underline{u}}=-\left(a_{1}(t)-|\Omega|\left|a_{2}(t)\right|-2 \chi\right)(\bar{u}-\underline{u}) \leq 0 .
$$

Thus by integrating over $\left(t_{0}, t\right)$, we get

$$
0 \leq \ln \frac{\bar{u}}{\underline{u}} \leq \ln \frac{\bar{u}_{0}}{\underline{u}_{0}}, \quad \text { and then } \frac{\bar{u}(t)}{\underline{u}(t)} \leq \frac{\bar{u}_{0}}{\underline{u}_{0}} .
$$

We have by mean value theorem that

$$
\bar{u}-\underline{u}=e^{\ln \bar{u}}-e^{\ln \underline{u}}=e^{\ln \hat{u}}\left(\ln \frac{\bar{u}}{\underline{u}}\right)=\hat{u}\left(\ln \frac{\bar{u}}{\underline{u}}\right),
$$

where $\underline{u} \leq \hat{u} \leq \bar{u}$. Therefore

$$
\frac{d}{d t}\left(\ln \frac{\bar{u}}{\underline{u}}\right) \leq-\left(a_{1}(t)-|\Omega|\left|a_{2}(t)\right|-2 \chi\right)\left(\inf _{t \geq t_{0}} \bar{u}(t) \frac{\underline{u}_{0}}{\bar{u}_{0}}\right)\left(\ln \frac{\bar{u}}{\underline{u}}\right) .
$$

By letting $\epsilon_{0}=\inf _{t \in \mathbb{R}}\left\{a_{1}(t)-|\Omega|\left|a_{2}(t)\right|-2 \chi\right\}\left(\inf _{t \geq t_{0}} \bar{u}(t) \frac{\underline{\underline{u}}_{0}}{\bar{u}_{0}}\right)$, we have $\epsilon_{0}>0$ and

$$
0 \leq \ln \frac{\bar{u}}{\underline{u}} \leq \ln \frac{\bar{u}_{0}}{\underline{u}_{0}} e^{-\epsilon_{0} t} \rightarrow 0 \quad \text { as } \quad t \rightarrow \infty .
$$

(2) Let $L_{1}(t)$ and $L_{2}(t)$ be as in (1.18) and (1.19), respectively. By (1.17),

$$
\mu=\limsup _{t-s \rightarrow \infty} \frac{1}{t-s} \int_{s}^{t}\left(L_{1}(\tau)-L_{2}(\tau)\right) d \tau<0
$$

Fix $0<\epsilon<-\mu$. Let $r_{1}$ and $r_{2}$ be as in (1.20) and (1.21), respectively. By (1.16), Theorem 1.5(1), and definition of $\mu$, for any $\epsilon>0$, there exists $T_{\epsilon}>0$ such that

$$
r_{2}-\epsilon \leq u\left(\cdot, t_{0}+t ; t_{0} ; u_{0}\right) \leq r_{1}+\epsilon, r_{2}-\epsilon \leq u^{*}(x, t) \leq r_{1}+\epsilon \forall x \in \bar{\Omega}, t \geq t_{0}+T_{\epsilon},
$$

and

$$
\int_{t_{0}}^{t_{0}+t}\left(L_{1}(s)-L_{2}(s)\right) d s \leq(\mu+\epsilon) t, \forall t_{0} \in \mathbb{R}, t \geq t_{0}+T_{\epsilon} .
$$


We first prove that for any entire positive solution $\left(u^{*}(x, t), v^{*}(x, t)\right)$ of (1.3), (1.22) holds. To simplify the notation, for given $t_{0} \in \mathbb{R}$ and $u_{0} \in C^{0}(\bar{\Omega})$ with $u_{0}(x) \geq 0$ and $u_{0}(\cdot) \neq 0$, set $u(t)=u\left(\cdot, t ; t_{0} ; u_{0}\right)$ and $u^{*}(t)=u^{*}(\cdot, t)$. Let $w(t)=u(t)-u^{*}(t)$. Then $w$ satisfy the equation

$$
\begin{aligned}
w_{t}= & \Delta w-\chi \nabla\left(w \cdot \nabla A^{-1} u\right)-\chi \nabla\left(u^{*} \cdot \nabla A^{-1} w\right)+w\left(a_{0}(t, x)-a_{1}(t, x)\left(u+u^{*}\right)\right. \\
& \left.-a_{2}(t, x) \int_{\Omega} u\right)-a_{2}(t, x)\left(\int_{\Omega} w\right) u^{*}
\end{aligned}
$$

for $t>t_{0}$. By the similar arguments for (4.6), we have that $\int_{\Omega} w_{+}^{2}$ is weakly differentiable and moreover

$$
\frac{d}{d t} \int_{\Omega} w_{+}^{2}=2 \int_{\Omega} w_{+} w_{t} \quad \forall \text { a.e. } t>t_{0} .
$$

Next, by multiplying (6.7) by $w_{+}$and integrating it over $\Omega$, we get

$$
\begin{aligned}
\frac{1}{2} \frac{d}{d t} \int_{\Omega} w_{+}^{2}+ & \int_{\Omega}\left|\nabla w_{+}\right|^{2}=\chi \int_{\Omega} w_{+} \nabla w_{+} \cdot \nabla A^{-1} u+\chi \int_{\Omega} u^{*} \nabla w_{+} \cdot \nabla A^{-1} w \\
& +\int_{\Omega} w_{+}^{2}\left(a_{0}(t, x)-a_{1}(t, x)\left(u+u^{*}\right)-a_{2}(t, x) \int_{\Omega} u\right)-\left(\int_{\Omega} w\right) \int_{\Omega} a_{2}(t, x) u^{*} w_{+}
\end{aligned}
$$

for a.e $t>t_{0}$. Integrating by part and using the equation of $A^{-1} u$, we get for a.e $t>t_{0}+T_{\epsilon}$

$$
\begin{aligned}
\frac{1}{2} \frac{d}{d t} \int_{\Omega} w_{+}^{2}+\int_{\Omega}\left|\nabla w_{+}\right|^{2}= & \frac{\chi}{2} \int_{\Omega} w_{+}^{2}\left(u-A^{-1} u\right)+\chi \int_{\Omega} u^{*} \nabla w_{+} \cdot \nabla A^{-1} w \\
& +\int_{\Omega} w_{+}^{2}\left(a_{0}(t, x)-a_{1}(t, x)\left(u+u^{*}\right)-a_{2}(t, x) \int_{\Omega} u\right) \\
& -\left(\int_{\Omega} w\right) \int_{\Omega} a_{2}(t, x) u^{*} w_{+} \\
\leq & \frac{\chi}{2} \int_{\Omega} w_{+}^{2}\left(u-A^{-1} u\right)+\chi \int_{\Omega} u^{*} \nabla w_{+} \cdot \nabla A^{-1} w \\
& +\int_{\Omega} w_{+}^{2}\left(a_{0, \text { sup }}-a_{1, \inf }(t)\left(u+u^{*}\right)-\left(a_{2, \inf }(t)\right)_{+} \int_{\Omega} u+\left(a_{2, \inf }(t)\right)_{-} \int_{\Omega} u\right) \\
& -\left(\int_{\Omega} w\right) \int_{\Omega} a_{2}(t, x) u^{*} w_{+} .
\end{aligned}
$$

We have by Young's inequality that

$$
\chi \int_{\Omega} u^{*} \nabla w_{+} \cdot \nabla A^{-1} w \leq \int_{\Omega}\left|\nabla w_{+}\right|^{2}+\frac{\left(\chi\left(r_{1}+\epsilon\right)\right)^{2}}{4} \int_{\Omega}\left|\nabla A^{-1} w\right|^{2} .
$$

Using the equation of $A^{-1} u$, we get

$$
\int_{\Omega}\left|\nabla A^{-1} w\right|^{2} \leq \int_{\Omega} w_{+}^{2}+\int_{\Omega} w_{-}^{2}
$$

for $t>t_{0}$. Also we have for $t>t_{0}+T_{\epsilon}$ that

$$
\begin{aligned}
& -\int_{\Omega} w \int_{\Omega} a_{2}(t, x) u^{*} w_{+} \\
& \leq \int_{\Omega} w_{-} \int_{\Omega}\left(a_{2, \sup }(t)\right)_{+} u^{*} w_{+}-\left(a_{2, \inf }(t)\right)_{+} \int_{\Omega} w_{+} \int_{\Omega} u^{*} w_{+}+\left(a_{2, \inf }(t)\right)_{-} \int_{\Omega} w_{+} \int_{\Omega} u^{*} w_{+} \\
& \leq\left[\left(r_{1}+\epsilon\right)\left(a_{2, \inf }(t)\right)_{-}-\left(r_{2}-\epsilon\right)\left(a_{2, \inf }(t)\right)_{+}\right]\left(\int_{\Omega} w_{+}\right)^{2}+\left(r_{1}+\epsilon\right)\left(a_{2, \sup }(t)\right)_{+}\left(\int_{\Omega} w_{-}\right)\left(\int_{\Omega} w_{+}\right) .
\end{aligned}
$$


By combining all these inequalities we have for a.e $t>t_{0}+T_{\epsilon}$ that

$$
\begin{aligned}
\frac{1}{2} \frac{d}{d t} \int_{\Omega} w_{+}^{2} \leq & \left(a_{0, \sup }(t)+\frac{\chi}{2}\left(\left(r_{1}+\epsilon\right)-\left(r_{2}-\epsilon\right)\right)+\frac{\left(\chi\left(r_{1}+\epsilon\right)\right)^{2}}{4}\right) \int_{\Omega} w_{+}^{2} \\
& -\left(\left(r_{2}-\epsilon\right)\left(2 a_{1, \inf }(t)+|\Omega|\left(a_{2, \inf }(t)\right)_{+}\right)\right) \int_{\Omega} w_{+}^{2} \\
& +2|\Omega|\left(r_{1}+\epsilon\right)\left(a_{2, \inf }(t)\right)_{-} \int_{\Omega} w_{+}^{2}+\left(\frac{\left(\chi\left(r_{1}+\epsilon\right)\right)^{2}}{4}\right) \int_{\Omega} w_{-}^{2} \\
& -\left(r_{2}-\epsilon\right)\left(a_{2, \inf }(t)\right)_{+}\left(\int_{\Omega} w_{+}\right)^{2}+\left(r_{1}+\epsilon\right)\left(a_{2, \sup }(t)\right)_{+}\left(\int_{\Omega} w_{-}\right)\left(\int_{\Omega} w_{+}\right) .
\end{aligned}
$$

Similarly we have that $\int_{\Omega} w_{-}^{2}$ is weakly differentiable with $\frac{d}{d t} \int_{\Omega} w_{-}^{2}=-2 \int_{\Omega} w_{-} w_{t}$, and for a.e $t>t_{0}+T_{\epsilon}$

$$
\begin{aligned}
\frac{1}{2} \frac{d}{d t} \int_{\Omega} w_{-}^{2} \leq & \left(a_{0, \sup }(t)+\frac{\chi}{2}\left(\left(r_{1}+\epsilon\right)-\left(r_{2}-\epsilon\right)\right)+\frac{\left(\chi\left(r_{1}+\epsilon\right)\right)^{2}}{4}\right) \int_{\Omega} w_{-}^{2} \\
& -\left(\left(r_{2}-\epsilon\right)\left(2 a_{1, \inf }(t)+|\Omega|\left(a_{2, \inf }(t)\right)_{+}\right)\right) \int_{\Omega} w_{-}^{2} \\
& +2|\Omega|\left(r_{1}+\epsilon\right)\left(a_{2, \inf }(t)\right)_{-} \int_{\Omega} w_{-}^{2}+\left(\frac{\left(\chi\left(r_{1}+\epsilon\right)\right)^{2}}{4}\right) \int_{\Omega} w_{+}^{2} \\
& -\left(r_{2}-\epsilon\right)\left(a_{2, \inf }(t)\right)_{+}\left(\int_{\Omega} w_{-}\right)^{2}+\left(r_{1}+\epsilon\right)\left(a_{2, \sup }(t)\right)_{+}\left(\int_{\Omega} w_{-}\right)\left(\int_{\Omega} w_{+}\right) .
\end{aligned}
$$

Note that

$$
\begin{aligned}
& -\left(r_{2}-\epsilon\right)\left(a_{2, \text { inf }}(t)\right)_{+}\left(\left(\int_{\Omega} w_{+}\right)^{2}+\left(\int_{\Omega} w_{-}\right)^{2}\right)+2\left(r_{1}+\epsilon\right)\left(a_{2, \sup }(t)\right)_{+}\left(\int_{\Omega} w_{-}\right)\left(\int_{\Omega} w_{+}\right) \\
& \leq 2\left(\left(r_{1}+\epsilon\right)\left(a_{2, \text { sup }}(t)\right)_{+}-\left(r_{2}-\epsilon\right)\left(a_{2, \text { inf }}(t)\right)_{+}\right)\left(\int_{\Omega} w_{-}\right)\left(\int_{\Omega} w_{+}\right) \\
& \leq|\Omega|\left[\epsilon\left(\left(a_{2, \text { sup }}(t)\right)_{+}+\left(a_{2, \text { inf }}(t)\right)_{+}\right)+\left(r_{1}\left(a_{2, \text { sup }}(t)\right)_{+}-r_{2}\left(a_{2, \text { inf }}(t)\right)_{+}\right)\right]\left(\int_{\Omega} w_{-}^{2}+\int_{\Omega} w_{+}^{2}\right) .
\end{aligned}
$$

Set

$K(t, \epsilon)=\chi \epsilon+\chi^{2} \frac{\epsilon}{2}\left(2 r_{1}+\epsilon\right)+2|\Omega| \epsilon\left(a_{2, \text { inf }}\right)_{-}+|\Omega| \epsilon\left(a_{2, \sup }(t)+\left(a_{2, \inf }(t)\right)_{+}\right)+\epsilon\left(2 a_{1, \inf }(t)+|\Omega| a_{2, \inf }(t)\right)$.

Adding (6.8) and (6.9), we then have

$$
\frac{d}{d t} \int_{\Omega}\left(w_{+}^{2}+w_{-}^{2}\right)(t) \leq 2\left\{L_{2}(t)-L_{1}(t)+K(t, \epsilon)\right\} \int_{\Omega}\left(w_{+}^{2}+w_{-}^{2}\right)
$$

for a.e $t>t_{0}+T_{\epsilon}$. Therefore by the continuity with respect to time of both sides of this last inequality, we get

$$
\frac{d}{d t} \int_{\Omega}\left(w_{+}^{2}+w_{-}^{2}\right)(t) \leq 2\left\{L_{2}(t)-L_{1}(t)+K(t, \epsilon)\right\} \int_{\Omega}\left(w_{+}^{2}+w_{-}^{2}\right)
$$

for $t>t_{0}+T_{\epsilon}$. Then by Gronwall's inequality,

$$
\int_{\Omega}\left(w_{+}^{2}(t)+w_{-}^{2}(t)\right) \leq \int_{\Omega}\left(w_{+}^{2}\left(t_{0}+T_{\epsilon}\right)+w_{-}^{2}\left(t_{0}+T_{\epsilon}\right)\right) e^{2 \int_{t_{0}}^{t}\left(L_{1}(s)-L_{2}(s)+K(s, \epsilon)\right) d s} \quad \text { for all } t>t_{0}+T_{\epsilon} .
$$


Note that $0 \leq \sup _{t \in \mathbb{R}}|K(t, \epsilon)| \rightarrow 0$ as $\epsilon \rightarrow 0$ and choose $\epsilon_{0} \ll 1\left(\epsilon_{0}<-\mu\right)$ such that

$$
0 \leq \sup _{t \in \mathbb{R}}|K(t, \epsilon)|<\frac{-\mu-\epsilon_{0}}{2}
$$

By $\int_{t_{0}}^{t}\left(L_{1}(s)-L_{2}(s)\right) d s \leq\left(\mu+\epsilon_{0}\right)\left(t-t_{0}\right)$ for $t \geq t_{0}+T_{\epsilon_{0}}$, we have

$$
\begin{aligned}
\int_{\Omega}\left(w_{+}^{2}(t)+w_{-}^{2}(t)\right) & \leq\left(\int_{\Omega} w_{+}^{2}\left(t_{0}+T_{\epsilon_{0}}\right)+w_{-}^{2}\left(t_{0}+T_{\epsilon_{0}}\right)\right) e^{2\left(\mu+\epsilon_{0}\right)\left(t-t_{0}\right)} e^{2\left(\frac{-\mu-\epsilon_{0}}{2}\right)\left(t-t_{0}\right)} \\
& \leq\left(\int_{\Omega} w_{+}^{2}\left(t_{0}+T_{\epsilon_{0}}\right)+w_{-}^{2}\left(t_{0}+T_{\epsilon_{0}}\right)\right) e^{\left(\mu+\epsilon_{0}\right)\left(t-t_{0}\right)} \quad \forall t>t_{0}+T_{\epsilon_{0}} .
\end{aligned}
$$

Therefore

$$
\lim _{t \rightarrow \infty}\left\|u\left(\cdot, t+t_{0} ; t_{0}, u_{0}\right)-u^{*}\left(\cdot, t+t_{0}\right)\right\|_{L^{2}(\Omega)}=\lim _{t \rightarrow \infty}\left\|w\left(t+t_{0}\right)\right\|_{L^{2}(\Omega)}^{2}=0
$$

uniformly in $t_{0} \in \mathbb{R}$.

We claim that (1.22) holds. Suppose by contradiction that there is $t_{0} \in \mathbb{R}$ such that $u\left(\cdot, t ; t_{0}, u_{0}\right) \nrightarrow u^{*}(\cdot, t)$ in $C^{0}(\bar{\Omega})$ as $t \rightarrow \infty$. Then there exists $\epsilon_{0}>0$ and a sequence $t_{n} \rightarrow \infty$ as $n \rightarrow \infty$ such that

$$
\left\|u\left(\cdot, t_{n} ; t_{0}, u_{0}\right)-u^{*}\left(\cdot, t_{n}\right)\right\|_{C^{0}(\bar{\Omega})}>\epsilon_{0} .
$$

Since $u\left(\cdot, t_{n} ; t_{0}, u_{0}\right), u^{*}\left(\cdot, t_{n}\right) \in C^{0}(\bar{\Omega})$ are uniformly bounded and equicontinuous, there exists up to subsequence $u^{1}, u_{*}^{1} \in C^{0}(\bar{\Omega})$ such that $u\left(\cdot, t_{n} ; t_{0}, u_{0}\right), u^{*}\left(\cdot, t_{n}\right)$ converges respectively to $u^{1}, u_{*}^{1}$ in $C^{0}(\bar{\Omega})$. Therefore by dominated convergence theorem, $u\left(\cdot, t_{n} ; t_{0}, u_{0}\right) \rightarrow u_{1}$ and $u^{*}\left(\cdot, t_{n}\right) \rightarrow u_{*}^{1}$ in $L^{2}(\Omega)$ as $t \rightarrow \infty$. This implies that

$$
\lim _{t_{n} \rightarrow \infty}\left\|u\left(\cdot, t_{n} ; t_{0}, u_{0}\right)-u^{*}\left(\cdot, t_{n}\right)\right\|_{L^{2}(\Omega)}=0 .
$$

Hence we have that $u^{1}=u_{*}^{1}$. But also from $\left\|u\left(\cdot, t_{n} ; t_{0}, u_{0}\right)-u^{*}\left(\cdot, t_{n}\right)\right\|_{C^{0}(\bar{\Omega})}>\epsilon_{0}$, we get as $n \rightarrow \infty,\left\|u^{1}-u_{1}^{*}\right\|_{C^{0}(\bar{\Omega})} \geq \epsilon_{0}$, which is a contradiction. Hence (1.22) holds.

Next, we prove that (1.3) has a unique entire positive solution. Suppose that $\left(u_{1}^{*}(x, t), v_{1}^{*}(x, t)\right)$ and $\left(u_{2}^{*}(x, t), v_{2}^{*}(x, t)\right)$ are two entire positive solutions of (1.3). We claim that $\left(u_{1}^{*}(x, t), v_{1}^{*}(x, t)\right) \equiv$ $\left(u_{2}^{*}(x, t), v_{2}^{*}(x, t)\right)$ for any $t \in \mathbb{R}$. Indeed, fix any $t \in \mathbb{R}$, by the arguments in the proof of (6.10),

$$
\left\|u_{1}^{*}(\cdot, t)-u_{2}^{*}(\cdot, t)\right\|_{L^{2}(\Omega)}=\left\|u\left(\cdot, t ; t_{0}, u_{1}^{*}\left(\cdot, t_{0}\right)\right)-u\left(\cdot, t ; t_{0}, u_{2}^{*}\left(\cdot, t_{0}\right)\right)\right\|_{L^{2}(\Omega)} \rightarrow 0 \quad \text { as } \quad t_{0} \rightarrow-\infty .
$$

This together with the continuity of $u_{i}^{*}(x, t)(i=1,2)$ implies that $u_{1}^{*}(x, t) \equiv u_{2}^{*}(x, t)$ and then $v_{1}^{*}(x, t) \equiv v_{2}^{*}(x, t)$. Hence (1.3) has a unique entire positive solution.

Assume now that $a_{i}(t, x) \equiv a_{i}(x)(i=0,1,2)$. By Theorem $1.3(3)$ and the uniqueness of entire positive solutions of (1.3), (1.3) has a unique positive steady state solution.

Assume that $a_{i}(t+T, x)=a_{i}(t, x)(i=0,1,2)$. By Theorem 1.3 (1) and the uniqueness of entire positive solutions of (1.3), (1.3) has a unique positive periodic solution with period $T$.

Finally assume that $a_{i}(t, x)(i=0,1,2)$ are almost periodic in $t$ uniformly with respect to $x \in \bar{\Omega}$. Let $\left(u^{*}(x, t), v^{*}(x, t)\right)$ be the unique positive solution of (1.3). We claim that $\left(u^{*}(x, t), v^{*}(x, t)\right)$ is almost periodic in $t$. Indeed, for any sequences $\left\{\beta_{n}^{\prime}\right\},\left\{\gamma_{n}^{\prime}\right\} \subset \mathbb{R}$, by the almost periodicity of $a_{i}(t, x)$ in $t$, there are subsequences $\left\{\beta_{n}\right\} \subset\left\{\beta_{n}^{\prime}\right\}$ and $\left\{\gamma_{n}\right\} \subset\left\{\gamma_{n}^{\prime}\right\}$ such that 


$$
\lim _{m \rightarrow \infty} \lim _{n \rightarrow \infty} a_{i}\left(t+\beta_{n}+\gamma_{m}, x\right)=\lim _{n \rightarrow \infty} a_{i}\left(t+\beta_{n}+\gamma_{n}, x\right)
$$

uniformly in $t \in \mathbb{R}$ and $x \in \bar{\Omega}$ for $i=0,1,2$. Let

$$
\hat{a}_{i}(t, x)=\lim _{n \rightarrow \infty} a_{i}\left(t+\beta_{n}, x\right), \quad \check{a}_{i}(t, x)=\lim _{m \rightarrow \infty} \hat{a}_{i}\left(t+\gamma_{m}, x\right), \quad \tilde{a}_{i}(t, x)=\lim _{n \rightarrow \infty} a_{i}\left(t+\beta_{n}+\gamma_{n}, x\right)
$$

for $i=0,1,2$. Observe that $\hat{a}_{i}(i=0,1,2), \check{a}_{i}(i=0,1,2)$, and $\tilde{a}_{i}(i=0,1,2)$ also satisfy the hypothesis (H1) in the introduction, and $\check{a}_{i}=\tilde{a}_{i}$ for $i=0,1,2$.

Without loss of generality, we may assume that $\lim _{n \rightarrow \infty}\left(u^{*}\left(\cdot, t+\beta_{n}\right), v^{*}\left(\cdot, t+\beta_{n}\right)\right)$ exists in $C^{0}(\bar{\Omega})$. Let

$$
\left(\hat{u}^{*}(x, t), \hat{v}^{*}(x, t)\right)=\lim _{n \rightarrow \infty}\left(u^{*}\left(\cdot, t+\beta_{n}\right), v^{*}\left(\cdot, t+\beta_{n}\right)\right) .
$$

Then $\left(\hat{u}^{*}(x, t), \hat{v}^{*}(x, t)\right)$ is an entire positive solution of (1.3) with $a_{i}(t, x)$ being replaced by $\hat{a}_{i}(t, x)(i=0,1,2)$.

We may also assume that $\lim _{n \rightarrow \infty}\left(\hat{u}^{*}\left(\cdot, t+\beta_{n}\right), \hat{v}^{*}\left(\cdot, t+\beta_{n}\right)\right)$ exists in $C^{0}(\bar{\Omega})$. Let

$$
\left(\check{u}^{*}(x, t), \check{v}^{*}(x, t)\right)=\lim _{n \rightarrow \infty}\left(\hat{u}^{*}\left(\cdot, t+\beta_{n}\right), \hat{v}^{*}\left(\cdot, t+\beta_{n}\right)\right) .
$$

Then $\left(\check{u}^{*}(x, t), \check{v}^{*}(x, t)\right)$ is an entire positive solution of (1.3) with $a_{i}(t, x)$ being replaced by $\check{a}_{i}(t, x)(i=0,1,2)$.

Furthermore, we may assume that $\lim _{n \rightarrow \infty}\left(\hat{u}^{*}\left(\cdot, t+\beta_{n}+\gamma_{n}\right), \hat{v}^{*}\left(\cdot, t+\beta_{n}+\gamma_{n}\right)\right)$ exists in $C^{0}(\bar{\Omega})$. Let

$$
\left(\tilde{u}^{*}(x, t), \tilde{v}^{*}(x, t)\right)=\lim _{n \rightarrow \infty}\left(\hat{u}^{*}\left(\cdot, t+\beta_{n}+\gamma_{n}\right), \hat{v}^{*}\left(\cdot, t+\beta_{n}+\gamma_{n}\right)\right) .
$$

Then $\left(\tilde{u}^{*}(x, t), \tilde{v}^{*}(x, t)\right)$ is an entire positive solution of (1.3) with $a_{i}(t, x)$ being replaced by $\tilde{a}_{i}(t, x)(i=0,1,2)$. By the uniqueness of entire positive solutions of (1.3) with $a_{i}(t, x)$ being replaced by $\tilde{a}_{i}(t, x)(i=0,1,2)$, we have that

$$
\left(\tilde{u}^{*}(x, t), \tilde{v}^{*}(x, t)\right)=\left(\check{u}^{*}(x, t), \check{v}^{*}(x, t)\right) \quad \forall x \in \bar{\Omega}, t \in \mathbb{R} .
$$

It then follows from $\check{a}_{i}=\tilde{a}_{i}$ for $i=0,1,2$ that

$$
\lim _{m \rightarrow \infty} \lim _{n \rightarrow \infty}\left(u^{*}\left(x, t+\beta_{n}+\gamma_{m}\right), v^{*}\left(x, t+\beta_{n}+\gamma_{m}\right)\right)=\lim _{n \rightarrow \infty}\left(u^{*}\left(x, t+\beta_{n}+\gamma_{n}\right), v^{*}\left(x, \beta_{n}+\gamma_{n}\right)\right)
$$

and hence $\left(u^{*}(x, t), v^{*}(x, t)\right)$ is almost periodic in $t$. The theorem is thus proved.

Remark 6.1. For the proof of Theorem 1.4 (2) in the general case of $\chi \in \mathbb{R}$, we need to see that

$$
\begin{aligned}
& \chi \int_{\Omega} w_{+}^{2}\left(u-A^{-1} u\right) \\
& =(\chi)_{+} \int_{\Omega} w_{+}^{2}\left(u-A^{-1} u\right)-(\chi)_{-} \int_{\Omega} w_{+}^{2}\left(u-A^{-1} u\right) \\
& \leq(\chi)_{+}\left(\left(r_{1}+\epsilon\right)-\left(r_{2}-\epsilon\right)\right) \int_{\Omega} w_{+}^{2}+(\chi)_{-}\left(-\left(r_{2}-\epsilon\right)+\left(r_{1}+\epsilon\right)\right) \int_{\Omega} w_{+}^{2}=\chi\left(\left(r_{1}+\epsilon\right)-\left(r_{2}-\epsilon\right)\right) \int_{\Omega} w_{+}^{2}
\end{aligned}
$$

and the proof then follows as in the case $\chi>0$. 


\section{References}

[1] S. Ahmad, Convergence and ultimate bounds of solutions of the nonautonomous VolterraLotka competition equations, J. Math. Anal. Appl., 127 (1987), no. 2, 377-387.

[2] H. Amann, Existence and regularity for semilinear parabolic evolution equations, Annali della Scuola Normale Superiore di Pisa, 11 (1984) 593-676.

[3] R.S. Cantrell and C. Cosner, The effects of spatial heterogeneity in population dynamics, J. Math. Biol. 29 (1991), no. 4, 315-338.

[4] M.A.J. Chaplain and J.I. Tello, On the stability of homogeneous steady states of a chemotaxis system with logistic growth term, Appl. Math. Lett., 57 (2016), 1-6.

[5] J.I. Diaz and T. Nagai, Symmetrization in a parabolic-elliptic system related to chemotaxis. Advances in Mathematical Sciences and Applications, Gakkotosho, Tokyo, 5 (1995), 659-680.

[6] A.M. Fink, Almost Periodic Differential Equations, Lectures Notes in Mathematics, Vol. 377, Springer-Verlag, Berlin-New York, 1974.

[7] R. Fisher, The wave of advance of advantageous genes, Ann. of Eugenics, 7(1937), 355-369.

[8] A. Friedman, Partial Differential Equations of Parabolic Types, Englewood Cliffs, NJ:Prentice-Hall, 1964.

[9] A. Friedman and J.I. Tello, Stability of solutions of chemotaxis equations in reinforced random walks, J. Math. Anal. Appl., 272 (2002), 138-163.

[10] H. Gajewski and K. Zacharias, Global behavior of a reaction-diffusion system modeling chemotaxis, Math. Nachr., 195 (1998), 177-194.

[11] D. Henry, Geometric Theory of Semilinear Parabolic Equations, Springer, Berlin, Heidelberg, New York, 1977.

[12] M.A. Herrero, E. Medina, and J.J.L. Velzquez, Singularity patterns in a chemotaxis model, Math. Ann., 306 (1996), 583-623.

[13] M.A. Herrero and J.J.L. Velzquez, A blow-up mechanism for a chemotaxis model, Ann. Sc. Norm. Super. Pisa, Cl. Sci., IV. Ser. 24 (1997), 633-683.

[14] M.A. Herrero and J.J.L. Velzquez, Finite-time aggregation into a single point in a reactiondiffusion system, Nonlinearity, 10 (1997), 1739-1754.

[15] P. Hess and H. Weinberger, Convergence to spatial-temporal clines in the Fisher equation with time-periodic fitness, J. Math. Biol. 28 (1990), 83-98.

[16] G. Hetzer and W. Shen, Convergence in almost periodic competition diffusion systems, $J$. Math. Anal. Appl. 262 (2001), 307-338.

[17] D. Horstmann, From 1970 until present: The Keller-Segel model in chemotaxis and its consequences, I. Jber. DMW, 105 (2003), 103-165.

[18] D. Horstmann and G. Wang, Blow-up in a chemotaxis model without symmetry assumptions, Eur. J. Appl. Math., 12 (2001), 159-177.

[19] D. Horstmann and M. Winkler, Boundedness vs.blow-up in a chemotaxis system, J. Differential Equations, 215 (2005), 52-107. 
[20] W. Jäger and S. Luckhaus, On explosions of solutions to a system of partial differential equations modeling chemotaxis, Trans. Amer. Math. Soc., 329 (1992), 819-824.

[21] E.F. Keller and L.A. Segel, Initiation of slime mold aggregation viewed as an instability, J. Theoret. Biol., 26 (1970), 399-415.

[22] E.F. Keller and L.A. Segel, A model for chemotaxis, J.Theoret. Biol., 30 (1971), 225-234.

[23] A. Khelghati and K. Baghaei, Global existence and boundedness of classical solutions in a quasilinear parabolic-elliptic chemotaxis system with logistic source, C. R. Math. Acad. Sci. Paris, 353 (2015), no. 10, 913-917.

[24] A. Kolmogorov, I. Petrowsky, and N.Piscunov, A study of the equation of diffusion with increase in the quantity of matter, and its application to a biological problem. Bjul. Moskovskogo Gos. Univ., 1 (1937), 1-26.

[25] A. Kubo and J.I. Tello, Mathematical analysis of a model of chemotaxis with competition terms, Differential Integral Equations, 29 (2016), no. 5-6, 441-454.

[26] K. Kuto, K. Osaki, T. Sakurai, and T. Tsujikawa, Spatial pattern formation in a chemotaxisdiffusion-growth model. Physica D 241 (2012), 1629-1639.

[27] J. Lankeit, Chemotaxis can prevent thresholds on population density. Discr. Cont. Dyn. Syst. B, 20 (2015), 1499-1527.

[28] J. Lankeit, Eventual smoothness and asymptotics in a three-dimensional chemotaxis system with logistic source. J. Differential Eq., 258 (2015), 1158-1191.

[29] T. Nagai, Blow-up of radially symmetric solutions of a chemotaxis system, Adv. Math. Sci. Appl., 5 (1995), 581-601.

[30] T. Nagai, Blowup of nonradial solutions to parabolic-elliptic systems modeling chemotaxis in two-dimensional domains, J. Inequal. Appl., 6 (2001), 37-55.

[31] M. Negreanu and J.I. Tello, On a competitive system under chemotaxis effects with nonlocal terms, Nonlinearity, 26 (2013), 1083-1103.

[32] M. Negreanu and J.I. Tello, Asysmptotic stability of a two species chemotaxis system with non-diffusive chemoattractant, J. Differential Eq., 258 (2015), 1592-1617.

[33] M.N. Nkashama, Dynamics of logistic equations with non-autonomous bounded efficients, Electronic Journal of Differential Equations, 2000 (2000), No. 02, 1-8.

[34] A. Pazy, Semigroups of Linear Operators and Applications to Partial Differential Equations, Applied Mathematical Sciences, 44. Springer-Verlag, New York, 1983.

[35] Painter, K.J., Hillen, T. Spatio-temporal chaos in a chemotaxis model. Physica D, 240(2011), 363-375

[36] T. Senba, Blowup Behavior of radial solutions to Jager-Luckhaus system in high dimensional domains, Funkcialaj Ekvacioj, 48 (2005), 247-271.

[37] T. Senba and T. Suzuki, Parabolic system of chemotaxis: blowup in a finite and the infinite time, Methods Appl. Anal., 8 (2001), 349-367.

[38] W. Shen and Y. Yi, Convergence in almost periodic Fisher and Kolmogorov models, J. Math. Biol. 37 (1998), no. 1, 84-102. 
[39] C. Stinner, J.I. Tello, and W. Winkler, Competive exclusion in a two-species chemotaxis, J.Math.Biol.. 68 (2014), 1607-1626.

[40] Y. Tao, M. Winkler Persistence of mass in a chemotaxis system with logistic source, $J$. DifferentialEquations, 259 (2015), 61426161.

[41] J.I. Tello, Mathematical analysis and stability of a chemotaxis problem with a logistic growth term, Math. Methods Appl. Sci., 27 (2004), 1865-1880.

[42] J.I. Tello and M.A.J. Chaplain, On the steady states and stability of solutions of a chemotaxis problem with a logistic growth term, Preprint.

[43] J.I. Tello and W. Winkler M, A chemotaxis system with logistic source, Common Partial Diff. Eq., 32 (2007), 849-877.

[44] J.I. Tello and M. Winkler, Stabilization in two-species chemotaxis with a logistic source, Nonlinearity, 25 (2012), 1413-1425.

[45] G. Viglialoro, Very weak global solutions to a parabolic-parabolic chemotaxis-system with logistic source, J. Math. Anal. Appl., 439 (2016), no. 1, 197-212.

[46] Y. Wang, A quasilinear attraction-repulsion chemotaxis system of parabolic-elliptic type with logistic source, J.Math. Anal.Appl., 441 (2016), 259-292.

[47] M. Winkler, Boundedness in the higher-dimensional parabolic-parabolic chemotaxis system with logistic source, Comm. Partial Differential Equations, 35 (2010), no. 8, 1516-1537.

[48] M. Winkler, Finite-time blow-up in the higher-dimensional parabolic-parabolic Keller-Segel system, J. Math. Pures Appl., 100 (2013), 748-767.

[49] M. Winkler, Blow-up in a higher-dimensional chemotaxis system despite logistic growth restriction, Journal of Mathematical Analysis and Applications, 384 (2011), 261-272.

[50] M. Winkler, How far can chemotactic cross-diffusion enforce exceeding carrying capacities? J. Nonlinear Sci., 24 (2014), 809-855.

[51] X.-Q. Zhao, Global attractivity in monotone and subhomogeneous almost periodic systems, J. Differential Equations, 187 (2003), 494-509.

[52] Q. Zhang, Y. Li. An attraction-repulsion chemotaxis system with logistic source, ZAMM - Journal of Applied Mathematics and Mechanics / Zeitschrift fr Angewandte Mathematik und Mechanik, 96(2016), 5, 570-584. 